\title{
Standard Problems for CFD Validation for NGNP - Status Report
}

Richard W. Johnson

Richard R. Schultz

August 2010

The INL is a

U.S. Department of Energy

National Laboratory

operated by

Battelle Energy Alliance

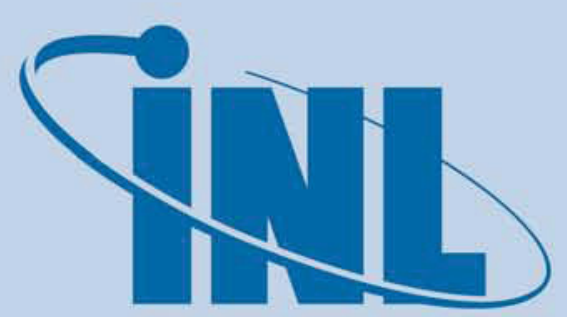

Idaho National Laboratory

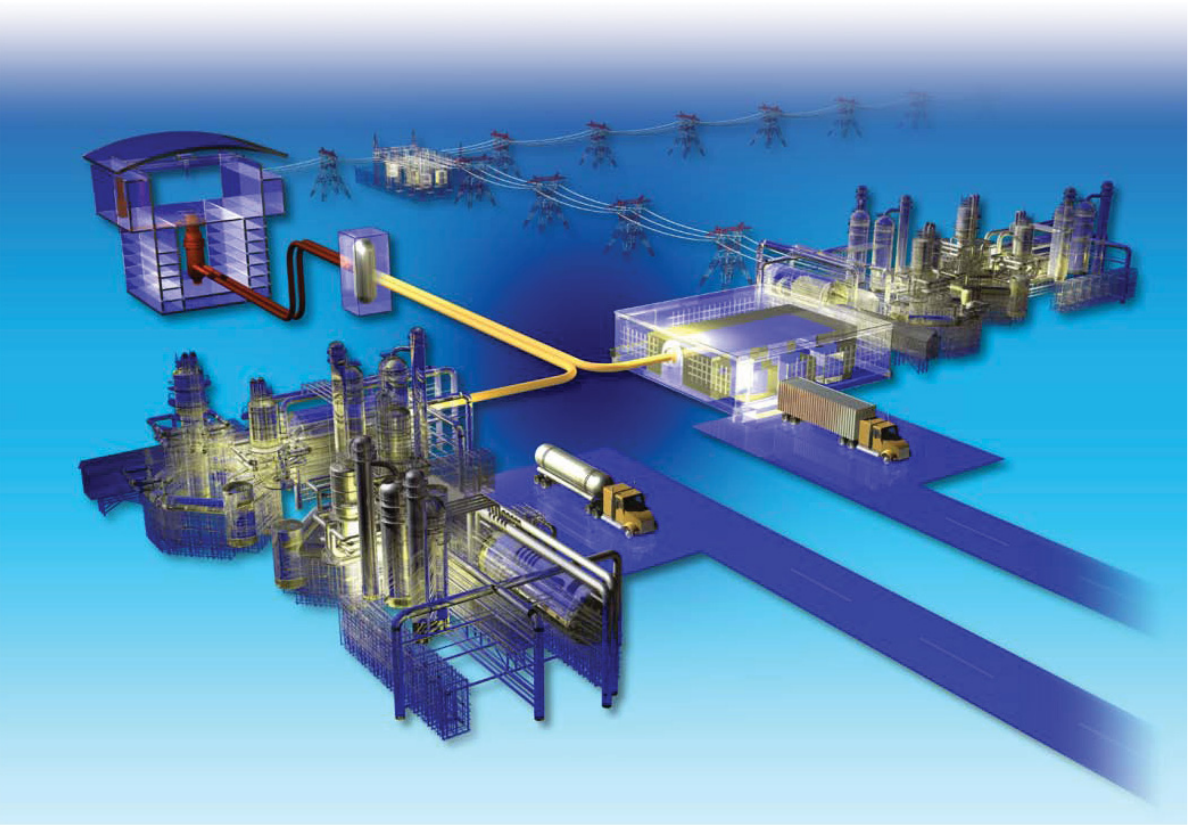




\section{DISCLAIMER}

This information was prepared as an account of work sponsored by an agency of the U.S. Government. Neither the U.S. Government nor any agency thereof, nor any of their employees, makes any warranty, expressed or implied, or assumes any legal liability or responsibility for the accuracy, completeness, or usefulness, of any information, apparatus, product, or process disclosed, or represents that its use would not infringe privately owned rights. References herein to any specific commercial product, process, or service by trade name, trade mark, manufacturer, or otherwise, does not necessarily constitute or imply its endorsement, recommendation, or favoring by the U.S. Government or any agency thereof. The views and opinions of authors expressed herein do not necessarily state or reflect those of the U.S. Government or any agency thereof. 


\title{
Standard Problems for CFD Validation for NGNP - Status Report
}

\author{
Richard W. Johnson
}

Richard R. Schultz

August 2010

Idaho National Laboratory

Next Generation Nuclear Plant Project

Idaho Falls, Idaho 83415

Prepared for the

U.S. Department of Energy

Office of Nuclear Energy

Under DOE Idaho Operations Office

Contract DE-AC07-05ID14517 



\title{
Standard Problems for CFD Validation for NGNP - Status Report
}

\author{
INL/EXT-10-19119
}

August 2010
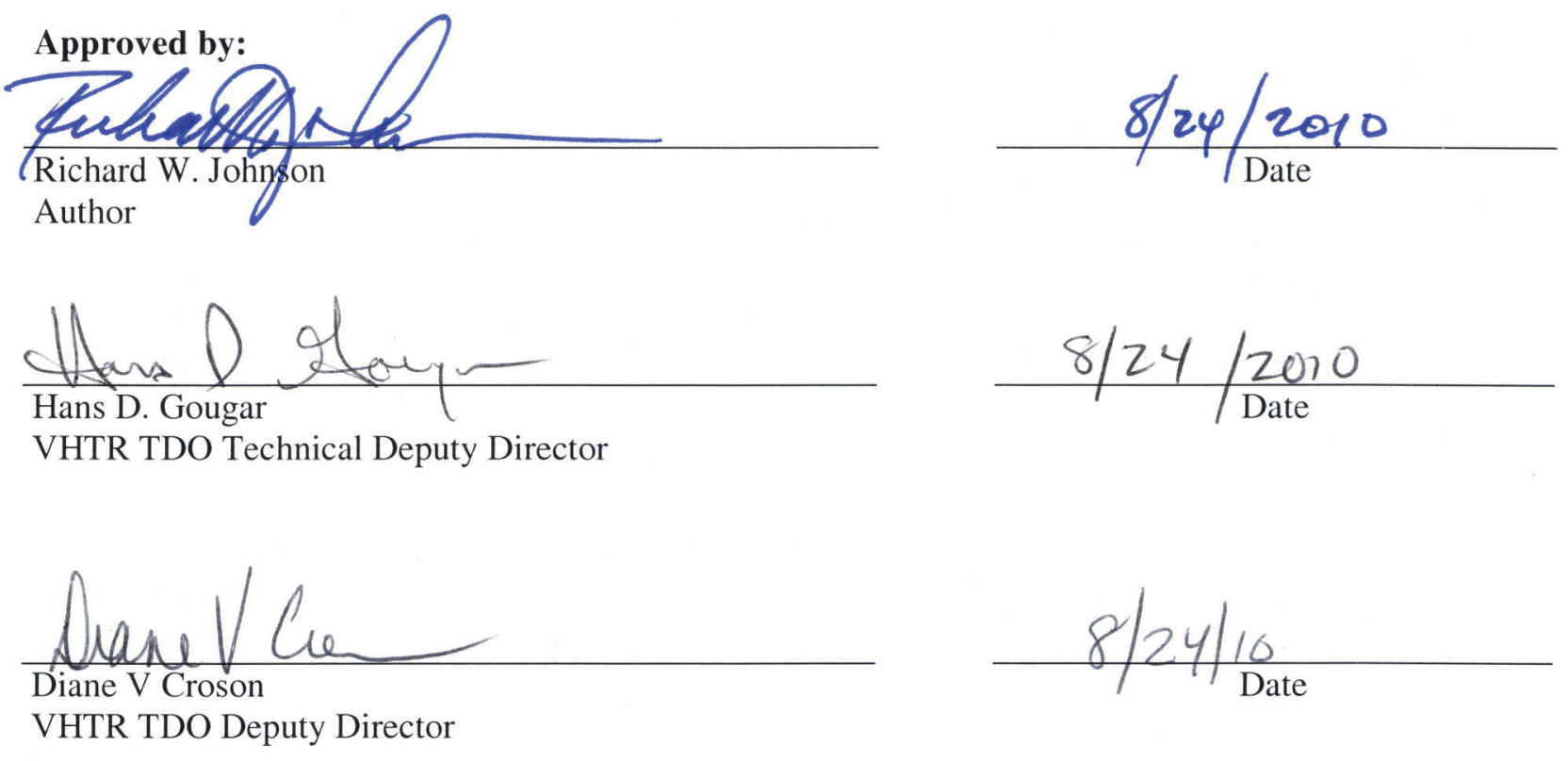


\section{SUMMARY}

The U.S. Department of Energy (DOE) is conducting research and development to support the resurgence of nuclear power in the United States for both electrical power generation and production of process heat required for industrial processes such as the manufacture of hydrogen for use as a fuel in automobiles. This work is being conducted under the Next Generation Nuclear Plant Project, which is based on a Generation IV very high temperature reactor (VHTR) concept. The VHTR will be of the prismatic or pebble bed type; the former is considered in this report. The VHTR will use helium as the coolant at temperatures ranging from $250^{\circ} \mathrm{C}$ to perhaps $1000^{\circ} \mathrm{C}$. While computational fluid dynamics (CFD) has not previously been used for the safety analysis of nuclear reactors in the United States, it is being considered for existing and future reactors. It is fully recognized that CFD simulation codes will have to be validated for flow physics reasonably close to actual fluid dynamic conditions expected in normal operational and accident situations. The "Standard Problem" is an experimental data set that represents an important physical phenomenon or set of phenomena, whose selection is based on a Phenomena Identification and Ranking Table for the reactor in question. Standard problems form the basis for determining whether a software tool is capable of analyzing the behavior of a reactor system undergoing review for an operating license. The term "Standard Problem" stems from the use of these data sets as a measure (hence, a standard) to determine the acceptability of the software. It will be necessary to build a database that contains a number of standard problems for use to validate CFD for the many physical problems that will need to be analyzed. It is expected that CFD computations will be performed to analyze reactor thermal fluid behavior in support of reactor design as well as for auditing and licensing calculations.

Two standard problems that have been developed consider flow in the lower plenum of the VHTR and bypass flow in the prismatic core. Both involve scaled models built from quartz and designed to be installed in the matched-index-of-refraction (MIR) test facility at Idaho National Laboratory (INL). The MIR facility employs mineral oil as the working fluid at a constant temperature. At this temperature, the index of refraction of the mineral oil is the same as that of the quartz. This provides an advantage to the optics used for data gathering. Particle image velocimetry (PIV) is used to take the data.

The objective of the present report is to present CFD calculations of the two standard problems for the purpose of investigating the experimental data to determine its suitability for use as validation data. That is, are there any problems with the data such as undesirable flow features, incomplete or inaccurate boundary conditions or problems with the geometry or experimental set up or locations of the data points?

The first standard problem represents several flow physics expected to be present in the lower plenum of the prismatic VHTR. In the lower plenum, heated helium coolant in the form of jets issues downward into the plenum and is then forced to turn 90 degrees and flow toward the exit duct. The lower plenum is filled with cylindrical graphite posts that hold up the core. Figure S-1 shows a plan view of the lower plenum. The red circles represent support posts holding up columns of heated blocks. Grey circles represent support posts under columns of reflector blocks. Helium enters the lower plenum at the junctions of the hexagonal blocks.

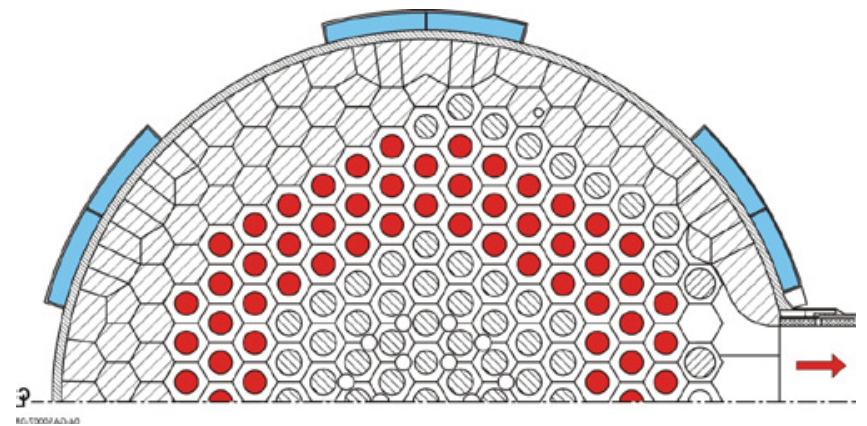

Figure S-1. Plan view of the VHTR lower plenum (figure courtesy of General Atomics). 
Geometry for the lower plenum standard problem is based on a narrow slice of the lower plenum and includes inlet jets, a portion of a hexagonal block that lines the wall of the lower plenum, and full and half cylinders representing the support posts. Figures S-2 and S-3 give isometric and plan views of the model, which is an exact scaled (1:6.55) replica of the narrow slice of the lower plenum. Because the model is an exact replica, half cylinders appear in the model. The wedge in Figure S-3 represents a graphite block lining the lower plenum.

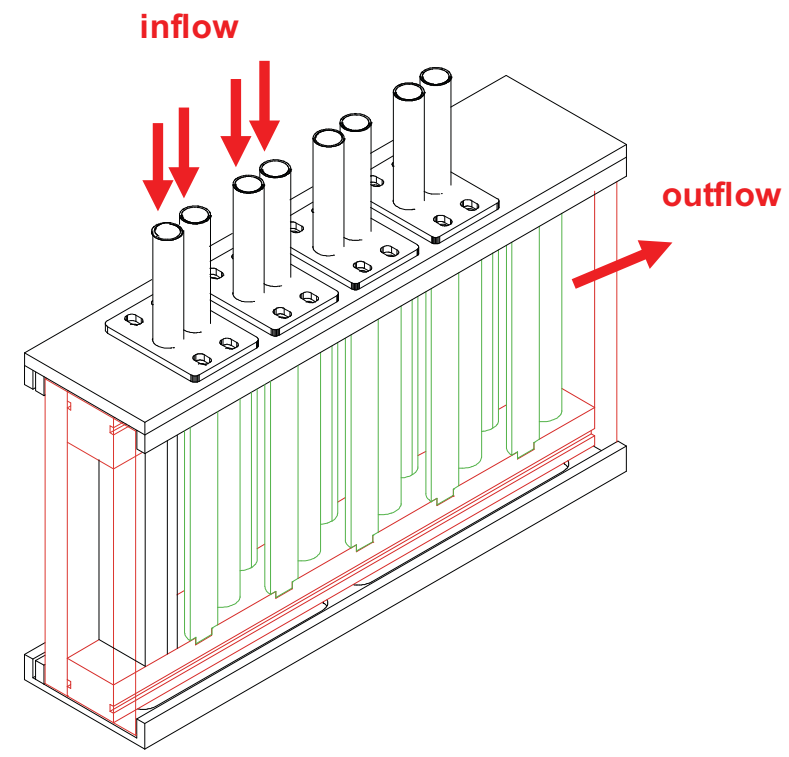

Figure S-2. Isometric view of the scaled model.

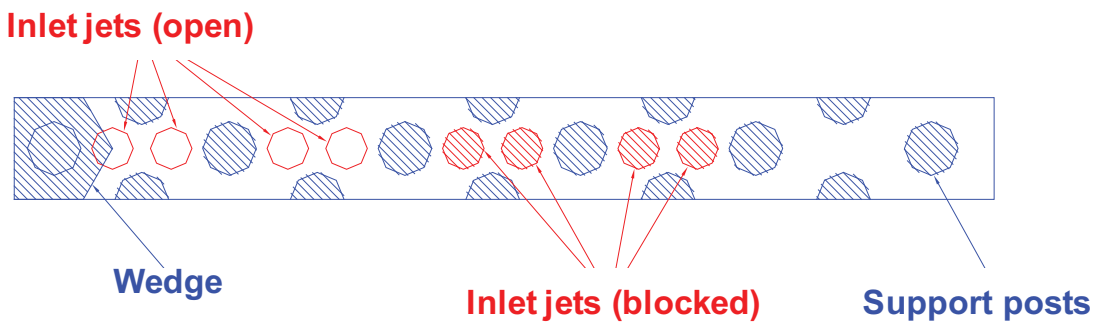

Figure S-3. Plan view of the scaled model.

PIV data were taken of the flow in the lower plenum quartz model. Extensive CFD simulations have been made of the flow and analyzed with the purpose of examining the data to determine if the data are suitable to be used for validation. While it may seem backwards to investigate the data based on CFD calculations, the simulations examine the nature of the flow and related aspects that may illuminate problems with the data. Two major problems have been found and are summarized here.

The first problem relates to the inlet flow of the mineral oil into the quartz model. This problem was reported last year, but is included for convenience. Basically, inlet jet flow was measured in two ways at the MIR test facility. First, the inlet flow was measured using four calibrated mass flow rotameters connected in-line with each inlet jet. Second, detailed inlet data were taken using PIV in the inlet ducts just above the inlet plane of the model. PIV data at 9.7 and $11 \mathrm{~mm}$ above the inlet plane were examined. These data were read into a commercial CFD code, interpolated onto the grid, and integrated by the code to calculate mass flow rates for each of the inlet jets. The flows measured by the rotameters are considered to be accurate, but it is clear that some error is present in the flow rates calculated from the 
PIV measurements. Some of this error is from the interpolation and integration processes. Table S-1 gives the percent differences in inlet mass flow for each inlet jet for the PIV data relative to the rotameter data. As can be seen, there are significant discrepancies between the measurements. The flow rates computed from the PIV data range from 1 to $25 \%$ lower than the rotameter data. While a discrepancy of 3 to $4 \%$ is acceptable, the data showing much higher differences are not. The source of these errors should be found and the PIV data retaken with the appropriate remedy.

Table S-1. Percent difference of mass flows of the PIV-based values relative to the rotameters.

\begin{tabular}{|l|c|c|c|c|c|}
\hline & Jet 1 & Jet 2 & Jet 3 & Jet 4 & Overall \\
\hline PIV $9.7 \mathrm{~mm}$ & $25.1 \%$ low & $7.98 \%$ low & $4.46 \%$ low & $8.99 \%$ low & $10.4 \%$ low \\
\hline PIV $11 \mathrm{~mm}$ & $18.1 \%$ low & $0.78 \%$ low & $2.99 \%$ low & $8.80 \%$ low & $6.70 \%$ low \\
\hline
\end{tabular}

The second problem found with the MIR data was discovered after extensive calculations. It was found that a recirculation zone (eddy) below the first jet is apparently randomly unstable, changing in size and causing dramatic excursions in the velocity data both near the inlet jets and downstream. Because the flow feature is changing randomly and because the time scale is relatively large, it is deemed that it is an undesirable flow feature to have in a validation data set. Figure S-4 shows the recirculation zone for calculations with all four inlet jets active at two times, indicating how the eddy changes dramatically. Also shown are time traces at a point below the third jet obtained from two calculations. These time traces exhibit large excursions of the vertical velocity values over time.
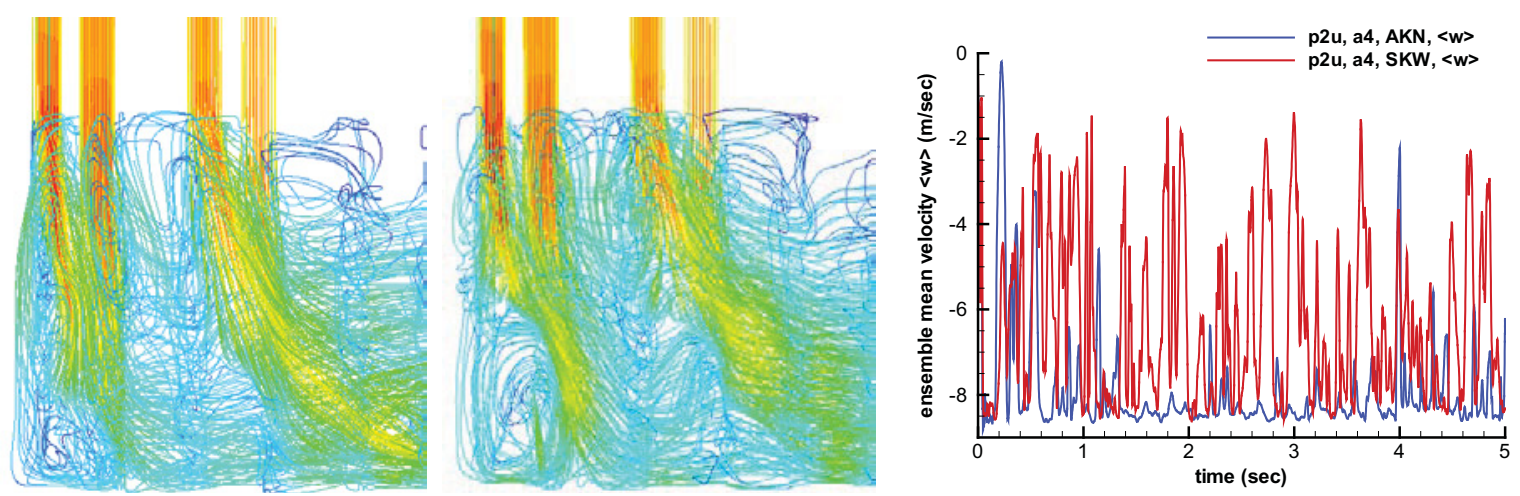

Figure S-4. Streamline plots showing lower-left eddy; time traces for all four jets active.

It was found from CFD calculations that the recirculation zone can be stabilized by eliminating the first jet. Figure S-5 plots the streamlines at two different times indicating an unchanging eddy. Also shown are time traces at the same point as in Figure S-4 for four turbulence models. The time traces eventually exhibit little or no fluctuation in the vertical velocity, indicating the flow is stabilized. It is recommended that the MIR data be retaken with improved inlet data and with the first jet turned off. 

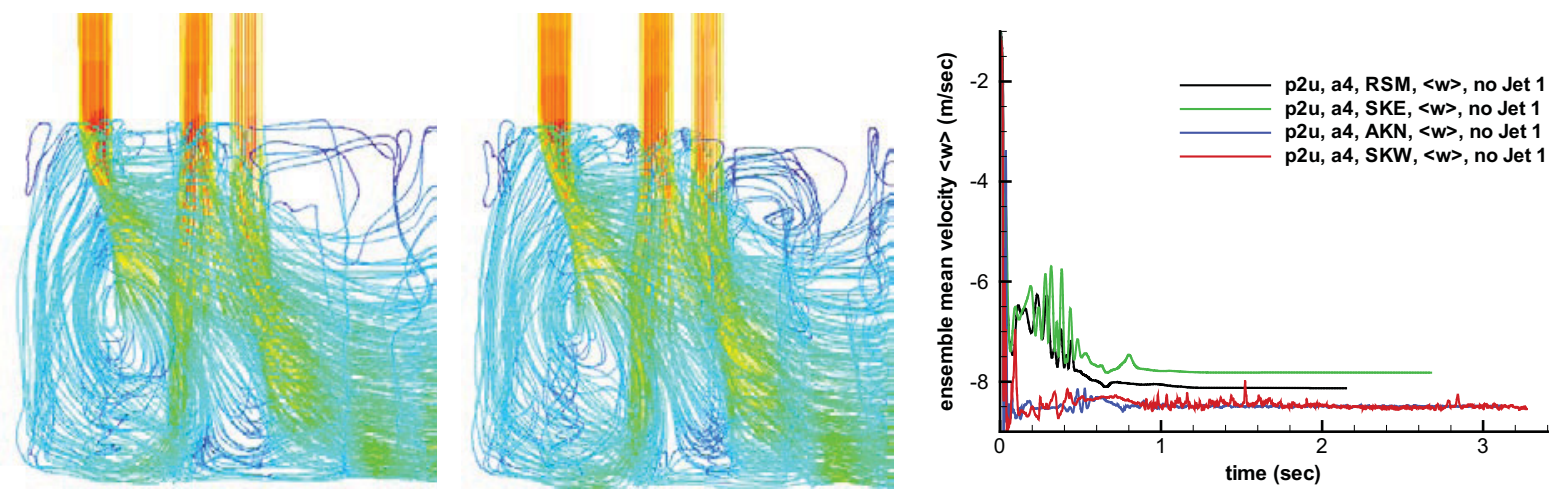

Figure S-5. Streamline plots showing eddy; time traces for the first jet turned off.

The bypass flow standard problem is concerned with the flow of coolant through the core that passes between the prismatic blocks. Gaps exist between the blocks because of tolerances in their size, imperfect installation and shrinkage caused by heating and irradiation. While bypass flow occurs elsewhere, the focus of the present standard problem is on the bypass flow between prismatic blocks. Figure S-6 shows a plan view of three adjacent prismatic fueled blocks. The model geometry is focused on the gap junction and is indicated by the thick red line in the figure, also shown in a close-up view.
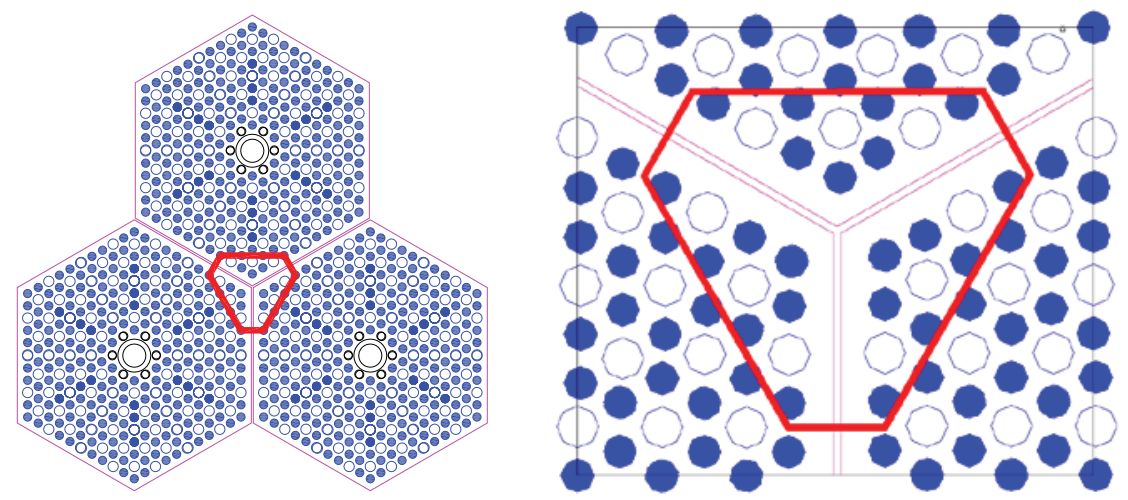

Figure S-6 Three prismatic blocks from the VHTR reactor showing the test model boundaries.

Figure S-7 provides a schematic of the bypass flow model to be installed in the MIR test facility. The model is scaled to be twice the size of the true geometry to make it easier to obtain measurements. The intent is to have one full-length fuel block section and a partial section with an adjustable horizontal gap between. The flow enters a side inlet and flows upwards into a dome which redirects it back downwards to an upper plenum before entering the nine channels and tri-gap region. The vertical gaps (those visible in Figure S-6) can be adjusted to three different spacings: 2, 6, and $10 \mathrm{~mm}$. It is anticipated that flow will be laminar, transitional, and turbulent for the 2, 6, and $10 \mathrm{~mm}$ gaps, respectively. Some pretest CFD calculations have been made of the flow in the bypass flow model. Figure S-8 illustrates the CFD model used. Only a one-sixth sector of the model geometry is used because of symmetry. 


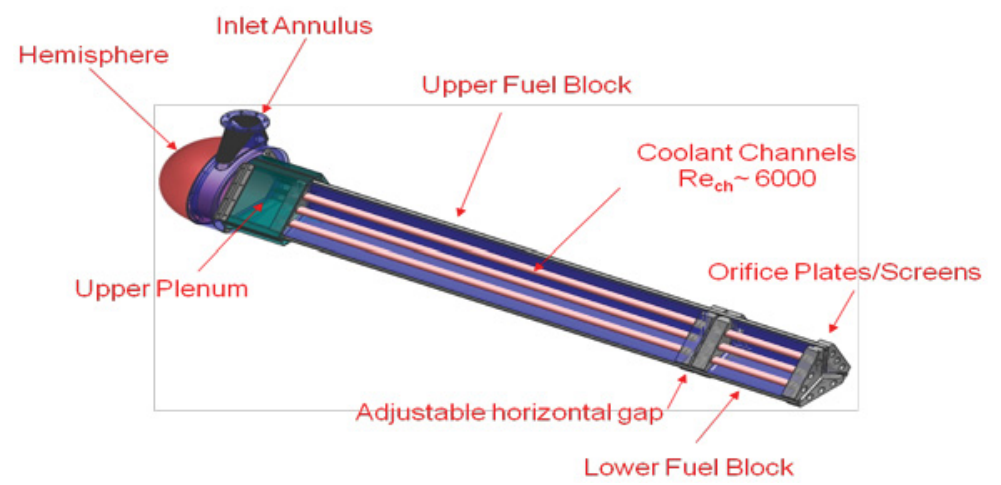

Figure S-7. Schematic of the bypass model.
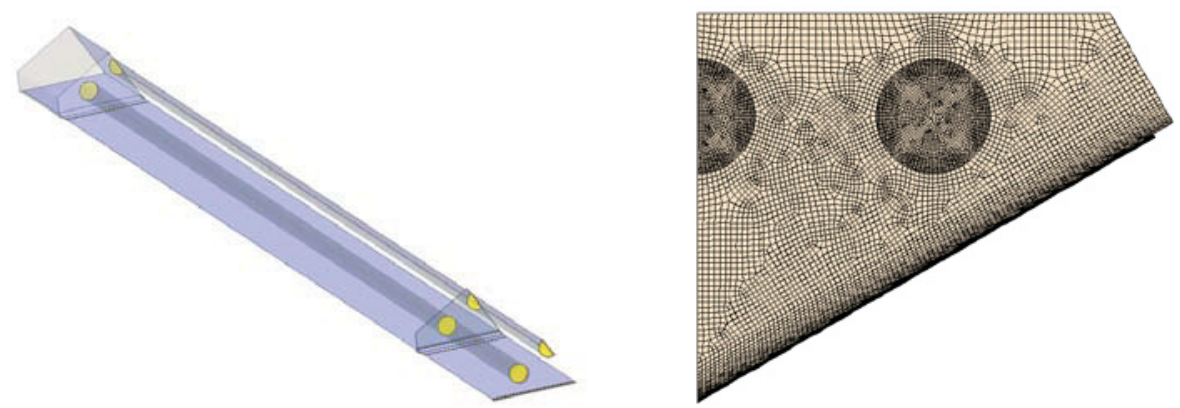

Figure S-8. Transparent view of the one-sixth sector CFD model and close-up of the end grid.

The flow in the $2 \mathrm{~mm}$ gap was found to be laminar. A close-up view of the gap flow in the upper fuel block can be seen in Figure S-9. It is apparent that the flow velocity increases somewhat in the center of the junction of the three gaps (at the lower left in the figure). However, it also appears to have little overall effect on the fluid dynamics. Figure S-10 shows a plot of pressure contours at the midpoint of the horizontal gap. As seen, the pressure is higher in the gap region, causing the flow to adjust toward the coolant channels. The flow in the gap in the lower fuel block is less than two-thirds that in the gap in the upper fuel block.

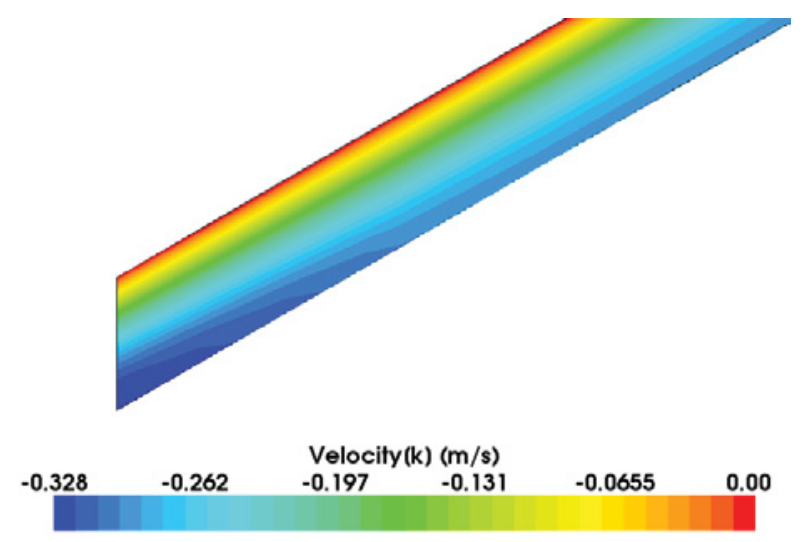

Figure S-9. Vertical velocity contours in the gap at the junction of the three blocks. 


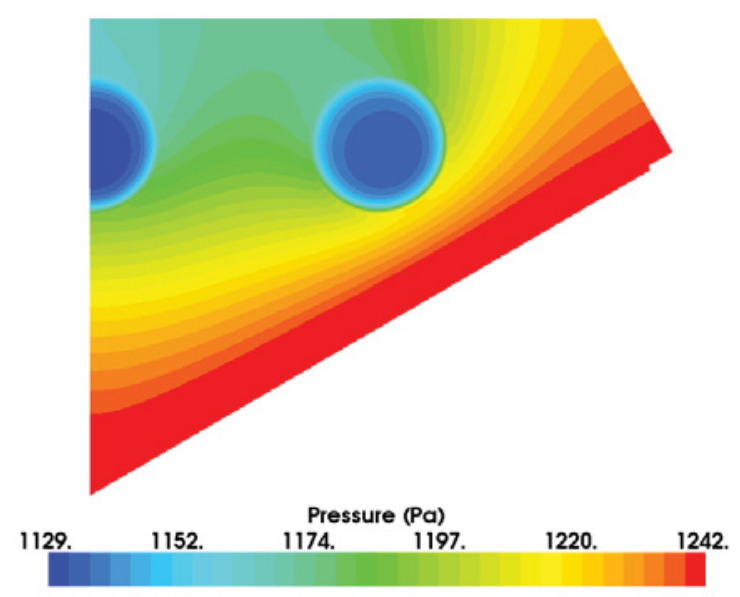

Figure S-10. Pressure contours in the mid-plane of the horizontal gap.

The CFD calculations will be compared to the MIR data for the bypass flow when it becomes available. Additional calculations will be made for the other vertical and horizontal gap spacings that will also be part of the experimental data matrix. The data will be examined to determine its suitability for use as a validation data set or standard problem. 


\section{CONTENTS}

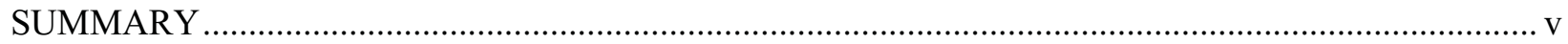

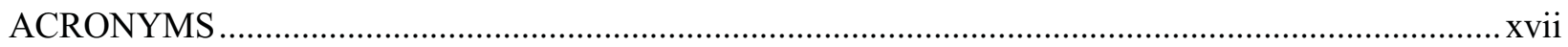

1. INTRODUCTION TO THE STANDARD PROBLEM …...................................................... 1

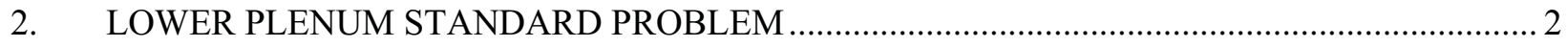

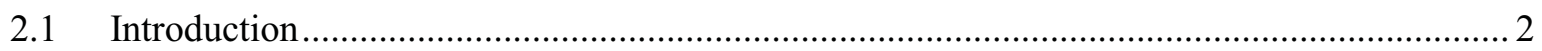

2.2 Nonstationary Turbulent Fluid Dynamics.................................................................... 3

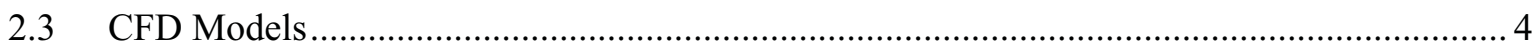

2.3.1 Flow Domain and Outlet Boundary Condition ..................................................... 4

2.3.2 Inlet Conditions........................................................................................ 6

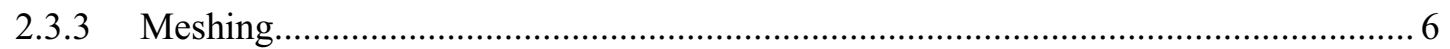

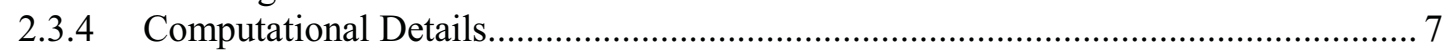

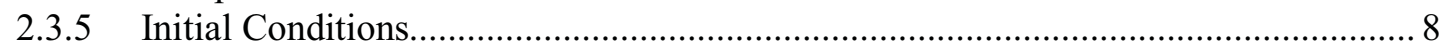

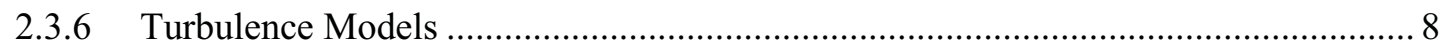

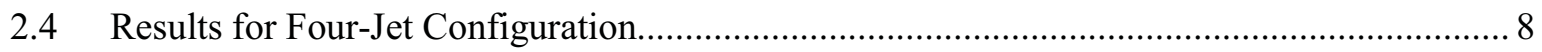

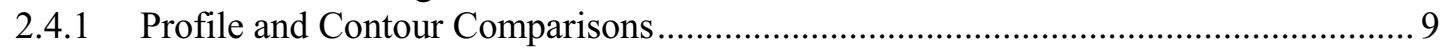

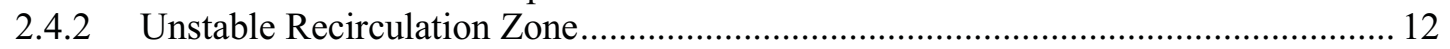

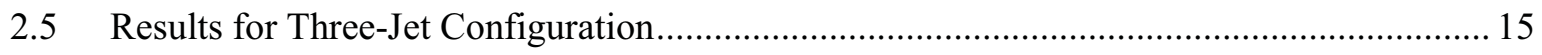

2.6 Summary and Recommendations for the Lower Plenum Standard Problem........................ 21

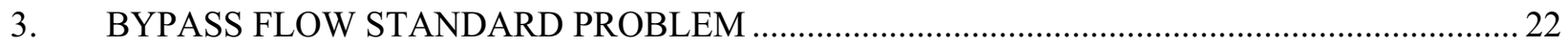

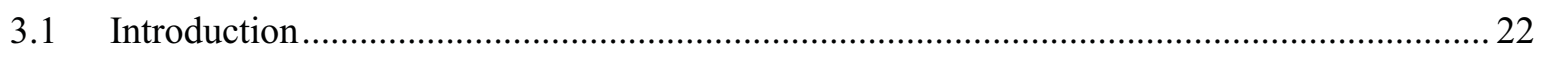

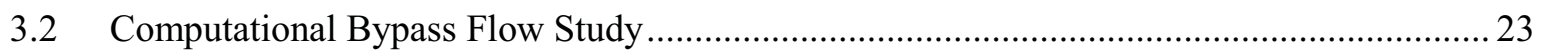

3.3 Bypass Flow Standard Problem Geometry .................................................................. 25

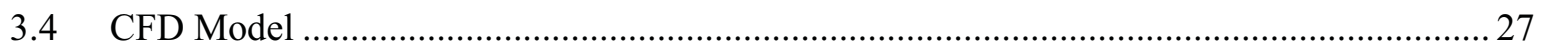

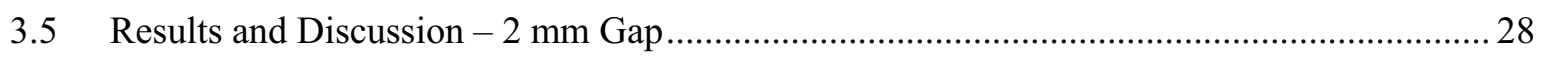

3.6 Summary of the Bypass Flow Standard Problem............................................................. 31

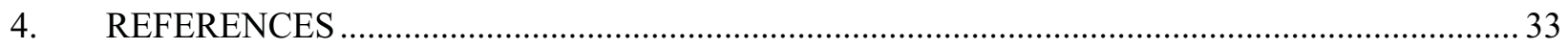

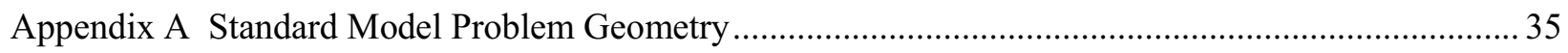

Appendix B Computer Code Quality Assurance ….......................................................................... 39

FIGURES

Figure S-1. Plan view of the VHTR lower plenum (figure courtesy of General Atomics)......................... V

Figure S-2. Isometric view of the scaled model.................................................................................... vi

Figure S-3. Plan view of the scaled model.......................................................................................

Figure S-4. Streamline plots showing lower-left eddy; time traces for all four jets active........................vii

Figure S-5. Streamline plots showing eddy; time traces for the first jet turned off. ................................viii

Figure S-6 Three prismatic blocks from the VHTR reactor showing the test model boundaries..............viii 


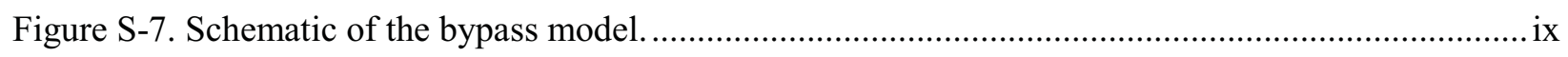

Figure S-8. Transparent view of the one-sixth sector CFD model and close-up of the end grid................ix

Figure S-9. Vertical velocity contours in the gap at the junction of the three blocks...............................ix

Figure S-10. Pressure contours in the mid-plane of the horizontal gap. ................................................

Figure 2-1. Plan view of the VHTR lower plenum (figure courtesy of General Atomics [GA])...............2

Figure 2-2. Isometric view of the scaled model and coordinate axes for the CFD model.......................... 3

Figure 2-3. Plan view of the scaled model..................................................................................... 3

Figure 2-4. Stream function contours of the flow in the CFD model that includes the outer flow.............. 4

Figure 2-5. Profiles of time mean velocity $U$ at (a) the outlet plane and at (b) at $3 \mathrm{f}$ in Figure 2-4............. 5

Figure 2-6. Profiles of (a) time mean velocity $V$ and (b) the Reynolds stress $\bar{u} \bar{v}$ at $3 \mathrm{f}$ in Figure 2-

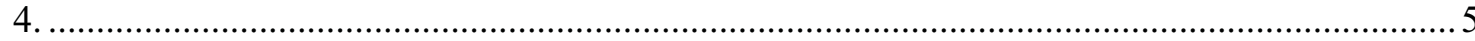

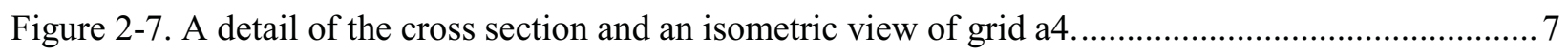

Figure 2-8. Locations of profiles and points for data comparison. ........................................................ 7

Figure 2-9. Time mean velocity $W$ along data profile ' $y 0 u$ ' at five different times. ................................. 8

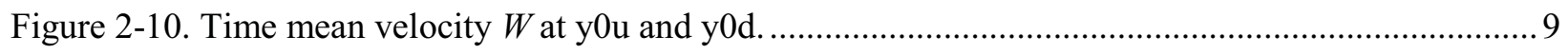

Figure 2-11. Contours of mean velocity $W$ at $Z=-0.07 \mathrm{~m}$ for (a) RSM, (b) SKE, (c) AKN and (d)

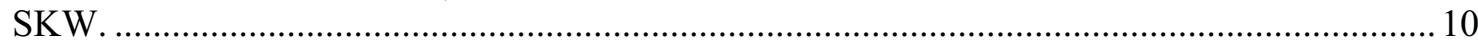

Figure 2-12. Contours of time mean velocity $W$ at $\mathrm{Z}=-0.07 \mathrm{~m}$ from the MIR data............................ 10

Figure 2-13. Comparison of time mean velocity $U$ results for the four cases with the MIR data. ............. 11

Figure 2-14. Time trace of ensemble average velocity $\langle u>$ at point $\mathrm{p} 7 \mathrm{~d}$ for the four turbulence

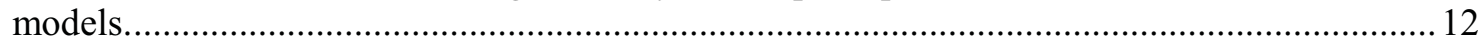

Figure 2-15. Time traces at points $\mathrm{p} 1 \mathrm{u}$ and $\mathrm{p} 2 \mathrm{u}$ for $\langle w>$ for the AKN and SKW models........................ 12

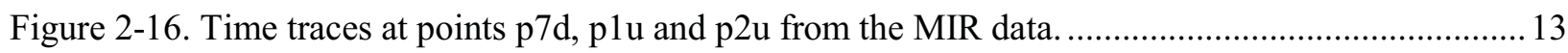

Figure 2-17. Turbulent to molecular viscosity ratio for the (a) RSM, (b) SKE, (c) AKN and (d) SKW.

Figure 2-18. Streamline plots for the SKW case at 3.6, 3.9 and 4.2 seconds, respectively...................... 14

Figure 2-19. Flow visualization photos of the flow at different times.................................................. 14

Figure 2-20. Time traces for points $\mathrm{p} 1 \mathrm{u}$ and $\mathrm{p} 2 \mathrm{u}$ for calculations for four models for the three-jet case.

Figure 2-21. Streamline plots at 2.7, 3.0, and 3.28 seconds for the three-jet case for the SKW model.

Figure 2-22. Trace of $\langle u\rangle$ at $\mathrm{p} 5 \mathrm{~d}$ for the three-jet configuration for the four turbulence models.

Figure 2-23. Time mean profiles for $W$ for stream-wise locations y0u and y0d for the three-jet results compared to the four-jet MIR data.

Figure 2-24. Contours of $W$ at $-0.07 \mathrm{~m}$ for the (a) RSM, (b) SKE, (c) AKN and (d) SKW models for the three-jet configuration. 
Figure 2-25. Profiles for $W$ for $\mathrm{x} 1 \mathrm{u}$ and $\mathrm{x} 1 \mathrm{~d}$ for the three-jet results compared to the four-jet MIR data.

Figure 2-26. Time mean profiles for $U$ for transverse locations $\mathrm{x} 1 \mathrm{u}$ and $\mathrm{x} 1 \mathrm{~d}$ for the three-jet results compared to the four-jet MIR data.

Figure 2-27. Time mean profiles for $U$ for transverse locations $\mathrm{x} 2 \mathrm{u}$ and $\mathrm{x} 2 \mathrm{~d}$ for the three-jet results compared to the four-jet MIR data.

Figure 2-28. Time mean profiles for $U$ for transverse locations $\mathrm{x} 3 \mathrm{u}$ and $\mathrm{x} 3 \mathrm{~d}$ for the three-jet results compared to the four-jet MIR data.

Figure 2-29. Time mean profiles for $U$ for transverse locations $\mathrm{x} 4 \mathrm{u}$ and $\mathrm{x} 4 \mathrm{~d}$ for the three-jet results compared to the four-jet MIR data.

Figure 2-30. Time mean profiles for $U$ for transverse locations $x 5 \mathrm{u}$ and $\mathrm{x} 5 \mathrm{~d}$ for the three-jet results compared to the four-jet MIR data.

Figure 2-31. Time mean profiles for $U$ for transverse locations x $6 \mathrm{u}$ and $\mathrm{x} 6 \mathrm{~d}$ for the three-jet results compared to the four-jet MIR data. 20

Figure 3-1 Schematic of bypass flow between prismatic blocks. .22

Figure 3-2. Plan view of a hexagonal block and one-twelfth symmetric sector. .23

Figure 3-3. Temperature contours for the 0,3 , and $5 \mathrm{~mm}$ gap cases for constant mass flow rate. .24

Figure 3-4 Three prismatic blocks from the MHTGR reactor and outline of test model geometry. .25

Figure 3-5. Close-up view of the origin of the bypass flow model and a cross-section view of the model. 26

Figure 3-6. Schematics of the bypass model showing components and flow paths. ..............................26

Figure 3-7 Plan view of the CFD model showing the mesh. ...............................................................2

Figure 3-8. Overview of CFD model and close-up of upper plenum of model.

Figure 3-9. Velocity profile of flow in the gap compared to the analytical solution for laminar flow. .29

Figure 3-10. Turbulent velocity profiles in the coolant channel in the first section. .29

Figure 3-11. Vertical velocity contours in the gap at the junction of the three blocks. ............................ 30

Figure 3-12. Axial pressure profiles through the full coolant channel and bypass flow gap..................... 30

Figure 3-13. Pressure contours in the mid-plane of the horizontal gap................................................. 31

Figure A-1. Top view of standard problem model geometry............................................................ 37

Figure A-2. Front view of standard problem model geometry. ........................................................... 38

\section{TABLES}

Table S-1. Percent difference of mass flows of the PIV-based values relative to the rotameters. vii

Table 2-1. Percent difference of mass flows from measured rotameter values for the PIV-based values. ... 6

Table 3-1. Inputs and results for the gap width study. .24

Table 3-2. Inputs and results for the $2 \mathrm{~mm}$ vertical gap studies. 
Table B-1. Quality assurance information for the computer codes used for the computer simulations.. 


\section{ACRONYMS}

AKN Abe, Kondoh, and Nagano ( turbulence model based on the $k \sim \varepsilon$ model and modified by researchers)

CFD computational fluid dynamics

DOE U. S. Department of Energy

GA General Atomics

GT-MHR Gas-Turbine Modular Helium Reactor

INL Idaho National Laboratory

MHTGR Modular High Temperature Gas Reactor

MIR matched-index-of-refraction

NGNP Next Generation Nuclear Plant

PIV particle image velocimetry

RANS Reynolds-averaged Navier-Stokes

RSM Reynolds stress transport turbulence model

SKE $\quad$ standard $k \sim \varepsilon$ turbulence model

SKW Menter shear stress transport $k \sim \omega$ turbulence model

URANS unsteady Reynolds-averaged Navier-Stokes

VHTR very high temperature reactor 
xviii 


\section{Standard Problems for CFD Validation for NGNP - Status Report}

\section{INTRODUCTION TO THE STANDARD PROBLEM}

The U.S. Department of Energy (DOE) is conducting research and development to support the resurgence of nuclear power in the United States for both electrical power generation and production of process heat required for industrial processes such as the manufacture of hydrogen for use as a fuel in automobiles. This work is being conducted under the Next Generation Nuclear Plant Project, which is based on a Generation IV very high temperature reactor (VHTR) concept. The VHTR will be either a prismatic or pebble bed type; the former is considered herein. The VHTR will use helium as the coolant at temperatures ranging from $250^{\circ} \mathrm{C}$ to perhaps $1000^{\circ} \mathrm{C}$. While computational fluid dynamics (CFD) has not previously been used for the safety analysis of nuclear reactors in the United States, it is being considered for existing and future reactors. It is fully recognized that CFD simulation codes will have to be validated for flow physics reasonably close to actual fluid dynamic conditions expected in normal operational and accident situations. The "Standard Problem" is an experimental data set that represents an important physical phenomenon or set of phenomena, whose selection is based on a Phenomena Identification and Ranking Table for the reactor in question. Standard problems form the basis for determining whether a software tool is capable of analyzing the behavior of a reactor system undergoing review for an operating license. The term "Standard Problem" stems from the use of these data sets as a measure (hence, a standard) to determine the acceptability of the software.

Standard problems consist of data sets that have the following characteristics: (1) the data set describes a phenomenon, or a set of phenomena, that influence the behavior of an important figure-ofmerit; (2) although a phenomenon may be measured in a reduced-scale system, it can be scaled to the fullsized system using accepted scaling practices; (3) the standard problem data set has uncertainties associated with each data point; (4) the quality assurance (QA) procedures used to design the experiment, build the experiment, and conduct the experiment are consistent with NQA-1 requirements (ASME 2000).

It will be necessary to build a database that contains a number of standard problems for use to validate CFD for the many physical problems that will need to be analyzed. It is expected that CFD computations will be performed to analyze reactor thermal fluid behavior in support of reactor design as well as for auditing and licensing calculations. 


\section{LOWER PLENUM STANDARD PROBLEM}

\subsection{Introduction}

Heated gas traverses the prismatic VHTR core before entering the lower plenum as jets where it mixes and exits the reactor vessel. The heat generation in the core is not uniform, leading to coolant jets of varying temperatures. Figure 2-1 gives a plan view of one-half of a reference prismatic VHTR lower plenum showing numerous cylindrical support pillars, locations of graphite blocks, and the exit duct. Jets enter the lower plenum at the corners of the heated hexagonal blocks (located above the red circles).

There are concerns that the heated helium may create hot spots in the lower plenum of the reactor vessel. There is also a concern that the helium will not be sufficiently mixed to attain a uniform temperature as it exits the reactor vessel. A standard problem that was designed to capture most of the flow physics present in the lower plenum was built and used to obtain validation data.

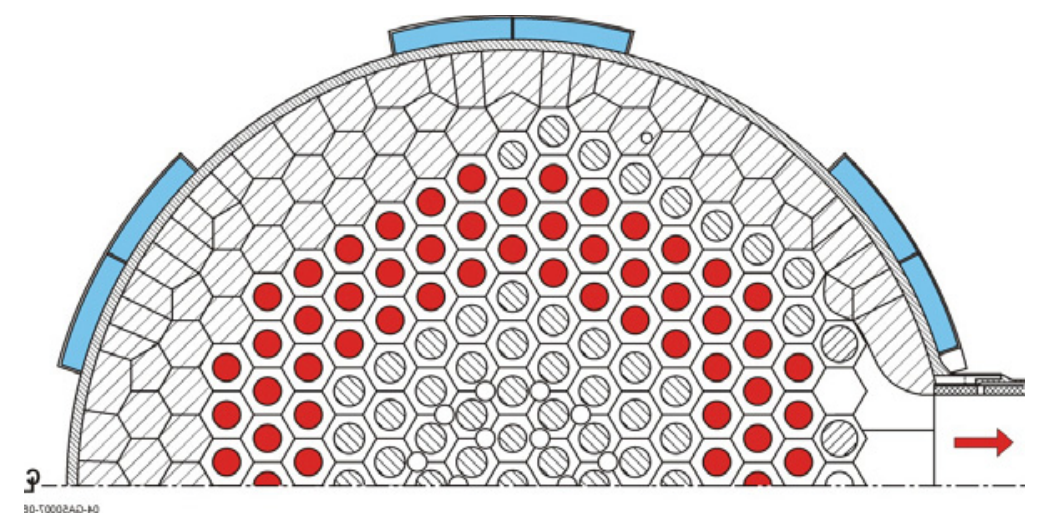

Figure 2-1. Plan view of the VHTR lower plenum (figure courtesy of General Atomics [GA]).

Experimental data were taken using stereo particle image velocimetry (PIV) in the matched-index-ofrefraction (MIR) facility at Idaho National Laboratory (INL) for a 1:6.55 scaled model of a narrow section of the lower plenum (Mcllroy et al. 2008). Figures 2-2 and 2-3 provide isometric and plan views of the scaled model, showing inlet ports, half and full cylindrical posts and the exit plane. The half cylinders are included in the scaled model because the model was designed to be an exact scaled replica. The inner model geometry is $53.98 \mathrm{~mm}$ wide, $558.8 \mathrm{~mm}$ long with the first jet located at $88.93 \mathrm{~mm}$ from the origin, and $217.5 \mathrm{~mm}$ high. The origin and coordinate axes are also given. Diameters for the inlet jets and posts are $0.0221 \mathrm{~mm}$ and $0.03175 \mathrm{~mm}$, respectively. Detailed drawings of the scaled model are provided in Appendix A.

A CFD study of the experimental data taken in the MIR was performed to examine the suitability of the data for use as a standard problem. Issues include the suitability of the model geometry, how many inlet jets should be employed, how sensitive the internal flow is to the symmetry/asymmetry of the inlet jets, and how complex the flow actually is. Other issues include the completeness and accuracy of the inlet conditions and whether the flow involves vortex-shedding. Three-dimensional (3-D) CFD calculations have been made to investigate these issues for a jet inlet Reynolds number of $\bar{u} d_{\text {jet }} / v=$ 12,400 , where $\bar{u}$ is the bulk velocity of the jet. The study employs Reynolds-averaged Navier-Stokes (RANS) turbulence models for the investigation of the MIR data.

The present report updates and extends CFD results and conclusions that were previously reported by Johnson and Schultz (2009). Details of the CFD model are repeated here for reader convenience. In particular, simulations are given using best inlet conditions for several turbulence models, and the presence of an undesirable flow feature in the flow as originally configured is identified. 
Recommendations are made to render moot the undesirable feature and improve the accuracy of the inlet data. New simulations are provided for the reconfigured experiment. The reconfiguration of the experiment is accomplished by simply shutting off the flow to the first jet (the one to the far left in Figures 2-2 and 2-3). The presence of the first jet gives rise to a recirculation zone (eddy) below the first and second jets, which exhibits a randomly unstable behavior that is undesirable for a data set to be used for validation purposes.

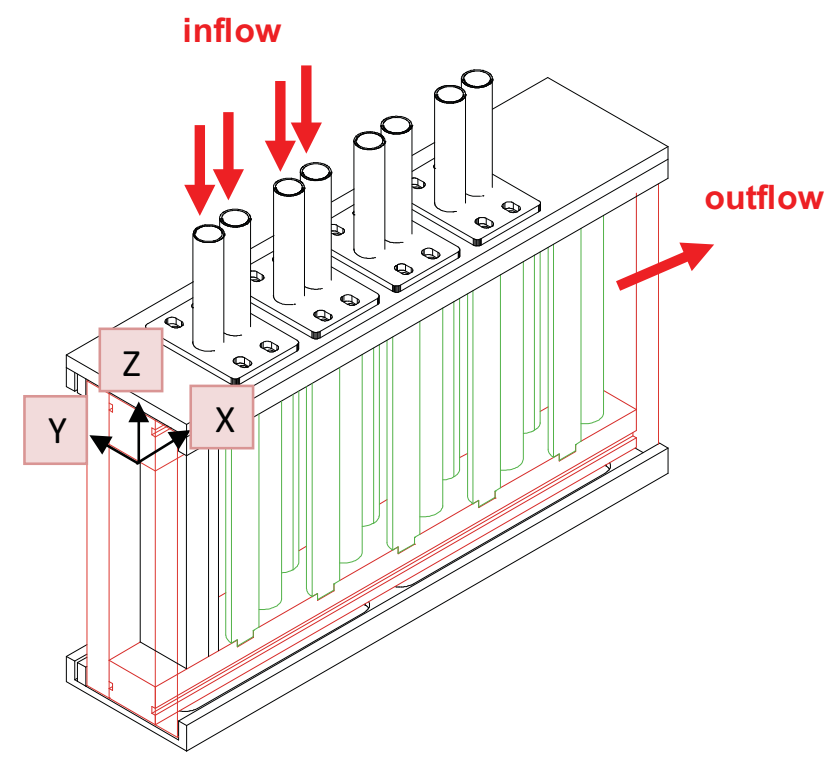

Figure 2-2. Isometric view of the scaled model and coordinate axes for the CFD model.

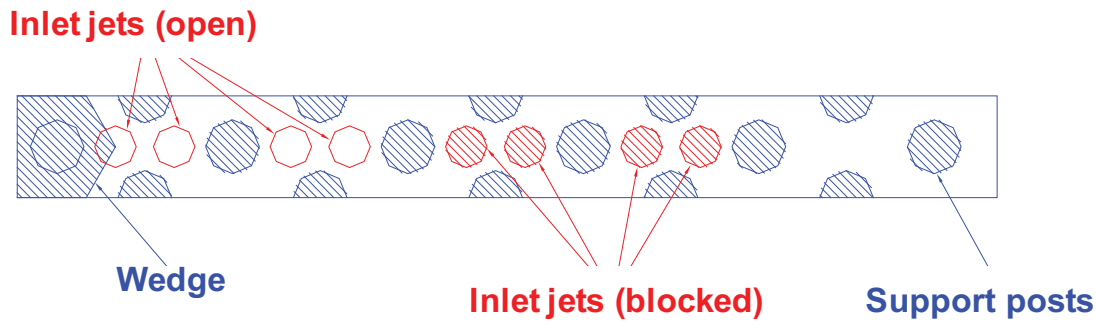

Figure 2-3. Plan view of the scaled model.

\subsection{Nonstationary Turbulent Fluid Dynamics}

Turbulent flow is characterized by apparently random fluctuations in all three spatial directions. In addition to these motions, there may be additional unsteady motions that are not from turbulence. Such flows are termed nonstationary. Examples of these include turbulent flow in turbomachinery where there is unsteadiness from rotating blades, turbulent flow around bluff bodies that causes vortex-shedding, and turbulent flow that is being pumped by a pulsating pump. In the present case, it is assumed that the flow around the cylindrical posts generates shedding vortices. For nonstationary turbulent flow, two averaging operations can be applied to the instantaneous flow quantities (Johnson 2008a). First, the random turbulence is averaged out of the Navier Stokes equations by performing an ensemble average. The procedure is called Reynolds averaging and the resulting equations are called the Reynolds-averaged Navier Stokes (RANS) equations. An approach that employs the unsteady RANS equations is called a URANS approach. Mathematically, for nonstationary flow, the instantaneous quantities are decomposed 
into an ensemble average and a random fluctuating component. For example, for the X-component velocity:

$u=\left\langle u>+u^{\prime}\right.$

where $\langle u\rangle$ is the ensemble average and $u^{\prime}$ is the turbulent fluctuation. The ensemble average can vary in time because of nonturbulent unsteadiness. The ensemble-averaged quantity can be decomposed further into a time-averaged component and a coherent fluctuation. The coherent fluctuation is related to nonturbulent unsteadiness such as vortex-shedding. For example,

$<u>=U+\widetilde{u}$

where $U$ is the time average of the ensemble average (or long-time average or just time mean) and $\tilde{u}$ is the coherent fluctuation. These averaging processes are applied to the velocity components and pressure. The velocity components are given as $u, v$, and $w$ in the $\mathrm{X}, \mathrm{Y}$, and $\mathrm{Z}$ directions, respectively, which are illustrated in Figure 2-2. The present CFD simulations compute the ensemble averages. These are concurrently time-averaged (by a running time-averaging calculation) because the experimental data of McIlroy, McEligot, and Pink (2008) are long-time averaged. Furthermore, the data were taken at a rate of 2 to 3 data planes per second, which is insufficiently rapid to capture either turbulent fluctuations or the vortex shedding. However, the data were taken for time intervals of about 375 seconds such that a fairly representative long-time average should have been obtained.

\subsection{CFD Models}

\subsubsection{Flow Domain and Outlet Boundary Condition}

The 104.8-mm-wide scaled model was positioned inside the test section of the INL's MIR facility, which has a cross section that is $609.6 \mathrm{~mm}$ square. The mineral oil working fluid not only flows into the scaled model, it also flows around the outside of the scaled model. A concern was that the outer flow would alter the flow at the exit of the scaled model, possibly affecting the flow inside the model. A 2-D study, using a URANS approach, was performed to assess the effects of the outer flow on the inner flow and determine if the outer flow should be included in the CFD model (Johnson 2008b). The commercial CFD code FLUENT v. 6.3.26 (see Appendix B) was used in the 2-D study. The Reynolds stress transport turbulence model (RSM) was employed. Mesh and iterative convergence were achieved.

Figure 2-4 illustrates the geometry and streamlines for the 2-D case where both inside and outside flows are included. As can be seen, the flow develops shedding vortices around the full and half cylinders inside the model; it can also be seen that there are vortices forming at the ends of the 25.4-mm-thick walls of the scaled model. It was found that because of the vortex shedding from the last cylinder, there is inflow at the exit plane of the scaled model.

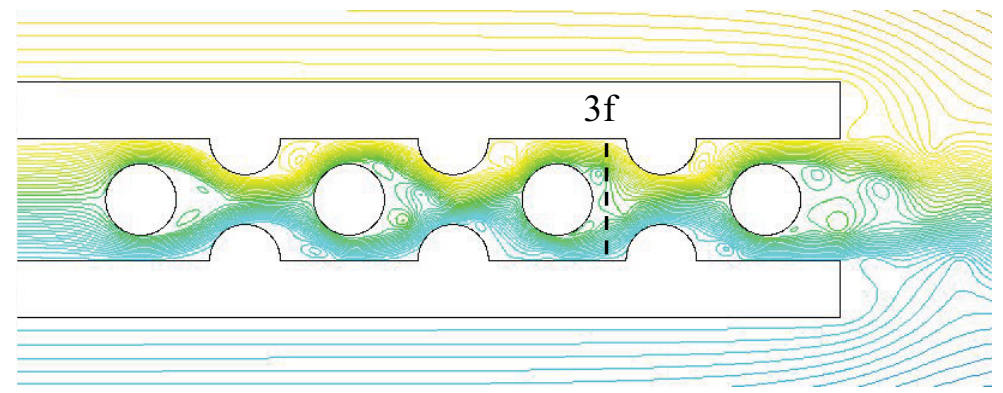

Figure 2-4. Stream function contours of the flow in the CFD model that includes the outer flow. 
Additional 2-D cases were run where only the inner flow was computed and two different boundary conditions were investigated at the outlet plane of the inner flow. The two boundary conditions used were the outflow and pressure-outlet conditions. These two boundary conditions are available in both commercial CFD codes FLUENT and STARCCM+ v. 3.04.20 (see Appendix B). The outflow boundary condition enforces a constant gradient condition for the velocity components while the pressure-outlet condition sets a constant static pressure just downstream of the outlet plane. Actually, neither of these conditions is exactly correct because of vortex shedding behind the last post.

Figure 2-5 illustrates the time mean stream-wise velocity $U$ at the outlet plane and at location $3 \mathrm{f}$, (see Figure 2-4), for cases employing the outflow and the pressure-outlet conditions compared to the reference case, which includes the outer flow (inner-outer). As shown, the three cases are distinctly different at the outlet plane. However, the pressure-outlet case compares very closely with the reference case at $3 \mathrm{f}$. Figure 2-6 compares the three cases for transverse velocity $V$ and the kinematic Reynolds stress $\bar{u} \bar{v}$ at $3 \mathrm{f}$. As shown, the pressure-outlet results closely match those for the reference case. These results provide confidence that including only the flow domain inside the scaled model and using the pressure-outlet condition will not cause inaccuracy in the results except in the region close to the outlet plane.

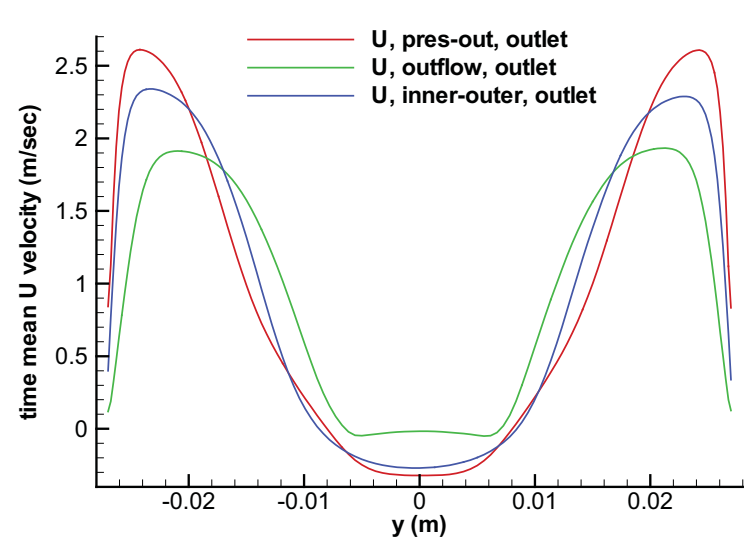

(a)

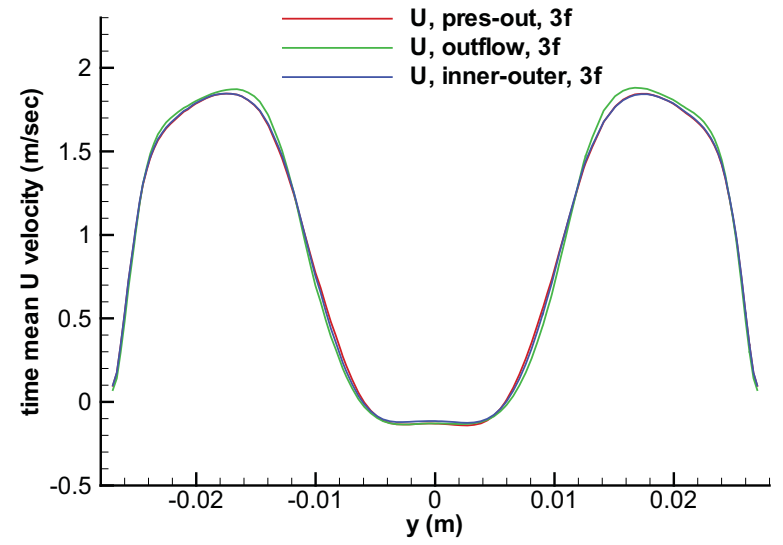

(b)

Figure 2-5. Profiles of time mean velocity $U$ at (a) the outlet plane and at (b) at $3 \mathrm{f}$ in Figure 2-4.

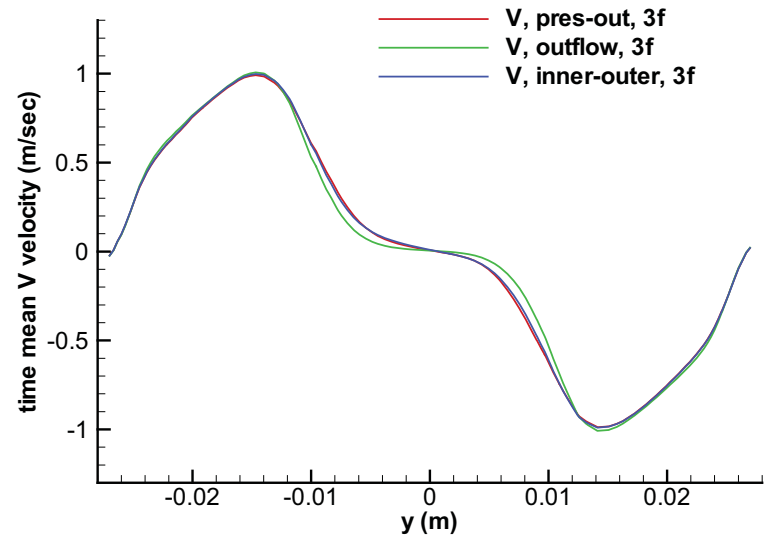

(a)

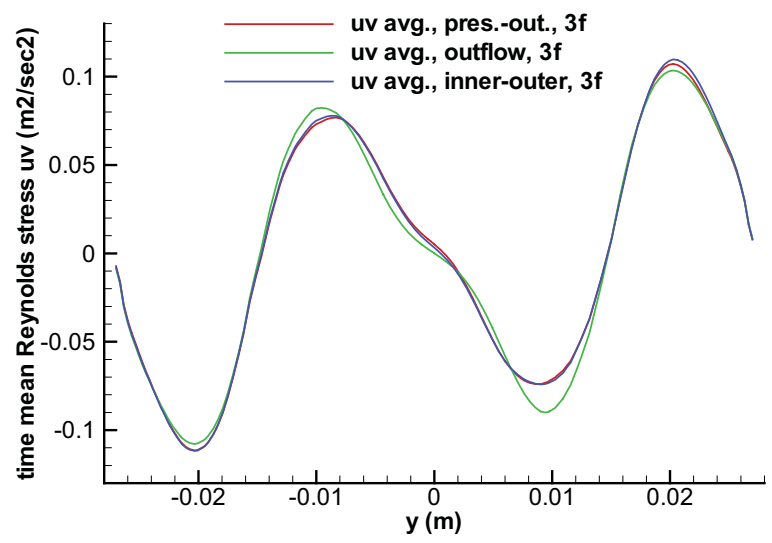

(b)

Figure 2-6. Profiles of (a) time mean velocity $V$ and (b) the Reynolds stress $\bar{u} \bar{v}$ at 3f in Figure 2-4. 


\subsubsection{Inlet Conditions}

Accurate and complete inlet boundary conditions are critical to the usefulness of any CFD validation data set. These include the velocity components and turbulence parameters, usually the turbulent kinetic energy. The inlet mass flows for the experiment were adjusted and measured by calibrated mass flow rotameters, one for each inlet duct. The mass flow to the first jet was set to be two-thirds that of the others because of the smaller flow area (see Figure 2-3). The rotameters provided accurate mass flow rates. Additionally, PIV data were taken for a vertical stretch of the inlet ducts of the scaled model. 3-D velocity data were taken in eleven $2 \mathrm{~mm}$ thick $X-Z$ planes illuminated by laser sheets. Data at $Z=9.7$ and $11 \mathrm{~mm}$ were extracted for use as inlet conditions.

The inlet data were read as tables into STARCCM+. Values were interpolated onto the grid and then integrated to obtain the mass flow rates. Unfortunately, the reduced PIV data do not generally compare well with the mass flow rotameter data (Johnson 2009). Table 2-1 provides information on the percent difference the mass flows based on the PIV data are from the rotameter data.

Table 2-1. Percent difference of mass flows from measured rotameter values for the PIV-based values.

\begin{tabular}{|l|c|c|c|c|c|}
\hline & Jet 1 & Jet 2 & Jet 3 & Jet 4 & overall \\
\hline PIV $9.7 \mathrm{~mm}$ & $25.1 \%$ low & $7.98 \%$ low & $4.46 \%$ low & $8.99 \%$ low & $10.4 \%$ low \\
\hline PIV $11 \mathrm{~mm}$ & $18.1 \%$ low & $0.78 \%$ low & $2.99 \%$ low & $8.80 \%$ low & $6.70 \%$ low \\
\hline
\end{tabular}

Clearly, there are some significant differences between the PIV-based data and the rotameter data for the mass flows, particularly for Jet 1 . Greater confidence in the PIV-measured inlet data would be obtained if the differences from the rotameter data were less than 5\%. This is actually true for Jet 3 at 9.7 and $11 \mathrm{~mm}$ and Jet 2 at $11 \mathrm{~mm}$. However, there are also significant differences between the PIV inlet data from 9.7 to $11 \mathrm{~mm}$ for Jets 1 and 2, which casts further doubt on the accuracy of the PIV-based inlet data. It was decided that the best approach for the inlet conditions is to use the rotameter data to compute bulk velocity in the Z-direction, set the other two velocities to zero, and match the turbulent kinetic energy in the core of the inlet ducts to the PIV data. The inlet data are applied at $\mathrm{Z}=88.5 \mathrm{~mm}$, which is the height of the inlet ducts. Just above this level are turbulence generating screens preceded by flow straighteners.

\subsubsection{Meshing}

In the aforementioned 2-D study (Johnson 2008b), three different grids of increasing fineness were employed. It was found that the intermediate and finest grid yielded very similar results. For the 3-D meshes, two 2-D grids were constructed based on the coarsest and intermediate 2-D grids above, then extruded vertically. Inlet ducts of $88.5 \mathrm{~mm}$ were added to the coarser grid, denoted a4. The number of cells in the Z-direction is 100 in the inlet ducts and 110 cells in the main body for a total of 5.8 million cells. Though it was intended to use the finer grid for final data comparison, it was decided to use the coarser grid for initial investigations of the MIR data because of excessive computing times. GAMBIT v. 2.4.6 (see Appendix B), which comes with FLUENT, was used to create the meshes. Figure 2-7 shows a detail of grid a 4 plus an isometric view. The inlet jet ports are green, the outlet plane is red and the walls are gray. 

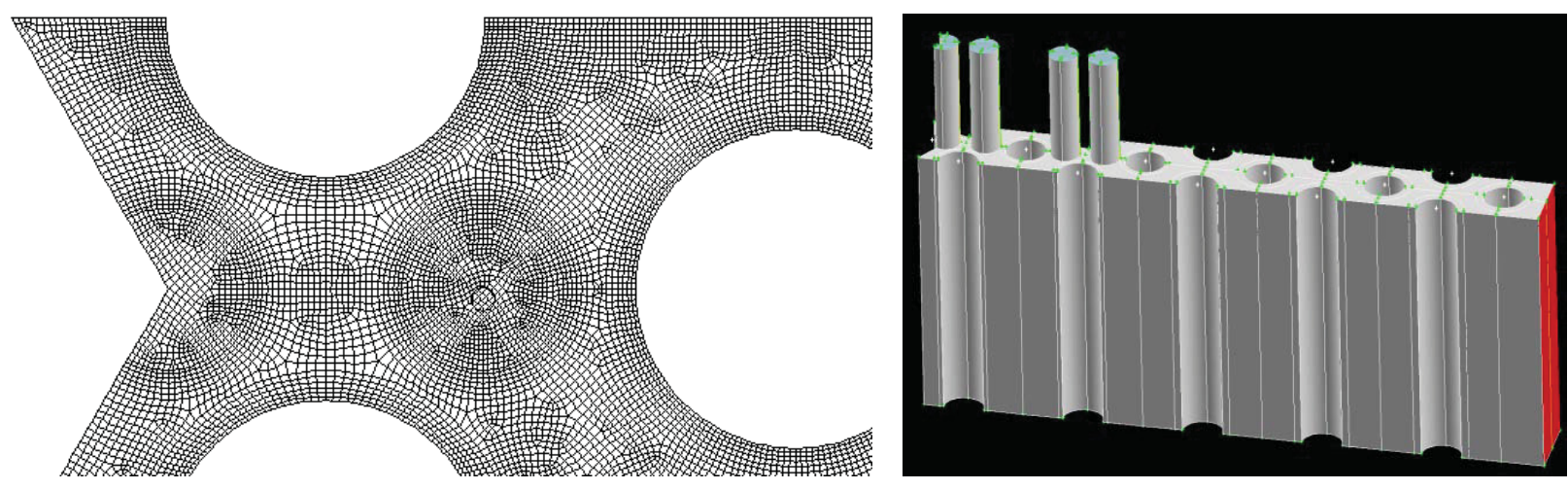

Figure 2-7. A detail of the cross section and an isometric view of grid a4.

\subsubsection{Computational Details}

The commercial CFD code STARCCM+ was used for the 3-D computations. The best practice guidelines used in the present study consist of those specified for the ASME Journal of Fluids

Engineering. ${ }^{\text {a }}$ The flow problem is set up using a URANS approach, because of the expectation of vortex shedding. Second-order differencing is used for both spatial and the implicit temporal discretization; the segregated solver is used. Walls are considered smooth and are assigned the no-slip condition.

Simulations use grid a4 and employ a constant time step of $2.0 \times 10^{-4}$ second. The 'pressure-outlet' boundary condition is used at the outlet as discussed above. The iterative convergence for each time step was set based on the calculation of a Poiseuille flow, which has an analytical solution. It was found from the Poiseuille flow calculation that a residual computed in STARCCM+ in the default mode is converged at a value of about $2 \times 10^{-4}$.

Figure 2-8 illustrates profile locations in the scaled model that will be referred to for data comparisons. The profiles shown in the plan view each represent two profiles: one at $Z=-0.07 \mathrm{~m}$ (given suffix ' $u$ ') and one at $Z=-0.15 \mathrm{~m}$ (suffix ' $d$ '). So, for example, profile ' $x 1 \mathrm{~d}$ ' is at location ' $x 1$ ' for $Z=$ $0.15 \mathrm{~m}$, while 'p2 $\mathrm{u}$ ' is at point $\mathrm{p} 2$ at $\mathrm{Z}=-0.07 \mathrm{~m}$.

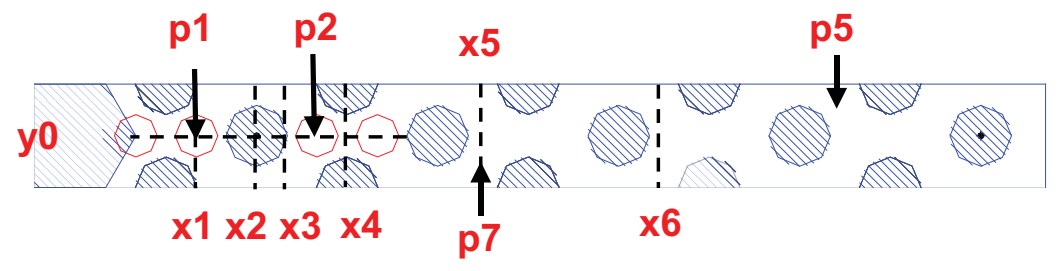

Figure 2-8. Locations of profiles and points for data comparison.

The CFD calculations must be long-time averaged to compare with the MIR data, which are averaged over 375 seconds. The long-time averaging is computed concurrently with the unsteady calculations. The question arises as to how long the simulation must be carried out to provide an unchanging long-time average. Figure 2-9 plots the time mean velocity $W$ along profile 'y0u,' for five points in time. Grid a4 and the Menter SST $k \sim \omega$ turbulence model and uniform inlet profiles are used. This profile captures the four jet inlets along the centerline. Computations for 2.4 seconds or less are significantly different from longer-time averages in the region of the first jet. These results actually indicate that there is some kind of instability in the vicinity of Jet 1 . This instability is investigated further below. It is concluded that calculations need to run for at least 3 seconds for the four-jet configuration.

a. http://journaltool.asme.org/Templates/JFENumAccuracy.pdf. 


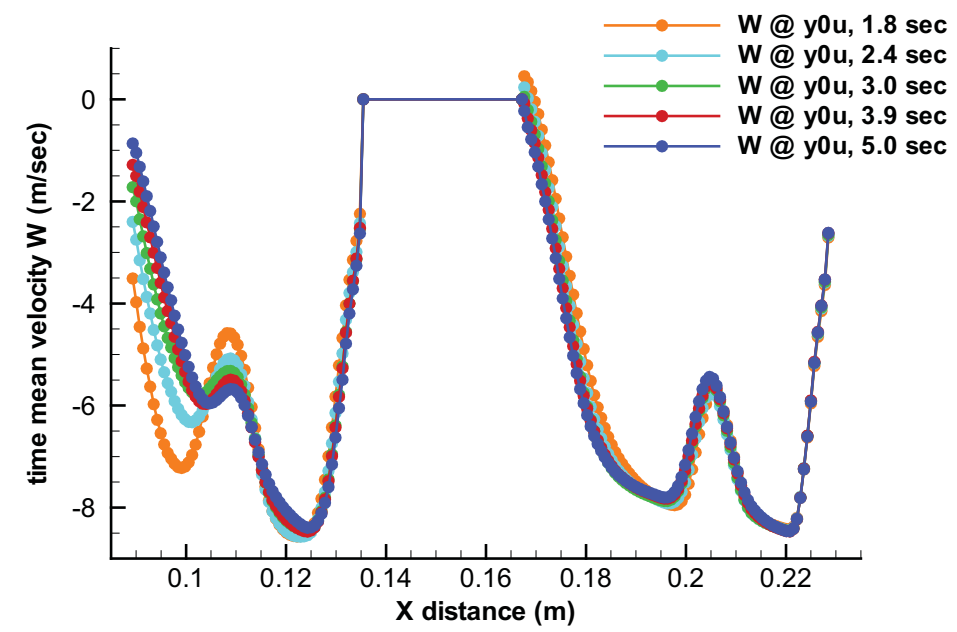

Figure 2-9. Time mean velocity $W$ along data profile 'y0u' at five different times.

\subsubsection{Initial Conditions}

Two methods for beginning the unsteady calculations were investigated. The first method is to perform a steady calculation until the residuals stop decreasing, then initiate unsteady calculations. The second is to start from a stagnant condition. Computations were made using the Abe-Kondoh-Nagano $(\mathrm{AKN}) k \sim \varepsilon$ turbulence model starting from these two initial conditions. Differences in results were not significant compared to differences using different turbulence models, Johnson and Schultz (2009).

\subsubsection{Turbulence Models}

RANS turbulent models are employed to represent the turbulence in the flow. Any turbulence model that is to be used for reactor safety analysis must be validated for the range of phenomena to which it is to be applied. The behavior of the turbulence model in the near-wall region is important because of its large effects on wall friction and heat transfer. This nonturbulent region has been found experimentally to be a region for which a dimensionless wall distance $y^{+}=y u_{\tau} / \rho<11$. Here $y$ is the dimensional distance, $u_{\tau}$ is the friction velocity defined as the square root of the ratio of wall shear stress to the density $\rho$. Some turbulence models require the additional specification of a wall treatment while others are designed to intrinsically apply all the way to the wall. The near-wall $y^{+}$values for grid a4 are mostly between 1 and 12 and therefore in the viscous region or sublayer. This is appropriate for turbulence models that are designed to handle the viscous sublayer. However, standard wall functions are also designed to work within the viscous sublayer. Four turbulence models are employed within STARCCM + in the present study. These are the Reynolds stress transport turbulence model (RSM) with the two layer all $y+$ wall treatment, the standard $k \sim \varepsilon$ (SKE) two layer model with the all $y^{+}$wall model, the Abe-Kondoh-Nagano (AKN) $k \sim \varepsilon$ low Re model with the all $y^{+}$wall model and the Menter shear-stress transport $k \sim \omega$ (SKW) with the all $y^{+}$wall model. The latter two turbulence models are specifically designed to apply up to the wall. The all $y^{+}$wall treatment is a hybrid treatment that uses wall functions if the near-wall node is outside the viscous sublayer, but uses the appropriate low $\mathrm{y}^{+}$treatment otherwise.

\subsection{Results for Four-Jet Configuration}

Results are obtained for the configuration that was employed in the MIR tests. For this configuration, four inlets are open and provide inlet flow to the scaled model. It will be shown that this configuration establishes a recirculation zone (eddy) below Jets 1 and 2 that is apparently randomly unstable, causing ensemble mean velocities to exhibit very large amplitude oscillations and undergo dramatic excursions to 
various time-local averages at random times. It was deemed appropriate to also perform computations with the first jet turned off to see if that configuration could lead to a stable recirculation zone.

Calculations for the four-jet configuration are first presented along with evidence suggesting a randomly unstable flow feature is present for this configuration. These will be compared to the MIR four-jet data. Later, simulations will be presented for a three-jet configuration.

\subsubsection{Profile and Contour Comparisons}

Results are obtained for the four-jet case employing the four turbulence models described above. The inlet conditions are based on the rotameter data as mentioned. The turbulent kinetic energy and turbulent dissipation rates are set to $0.1 \mathrm{~J} / \mathrm{kg}$ and $1.0 \mathrm{~J} / \mathrm{kg}$-sec for all jets for the RSM, SKE and AKN models. For the SKW, the inlet turbulent kinetic energy is the same while the specific dissipation rate is set to $1.0 \mathrm{sec}^{-1}$. All cases use grid a4 and are computed to 5 seconds, except for the SKE case, which becomes periodic and is cut off at 3.7 seconds. The long-time averages are performed over the five seconds, except for SKE, where it is performed over four periodic cycles.

Figure 2-10 plots time mean results for vertical velocity $W$ for the four turbulence models for profiles $\mathrm{y} 0 \mathrm{u}$ and $\mathrm{y} 0 \mathrm{~d}$. The four jet signatures are clearly visible in the data at y0u. Turbulence models AKN and SKW exhibit distinct signatures for Jet 1, but in the wrong place; The other two models do not exhibit signatures for Jet 1 . The calculations improve for the jets farther downstream until they match the signature for Jet 4 for all four turbulence models. Calculations for profile y0d are quite similar for all cases, though the signature for Jet 1 is missing.
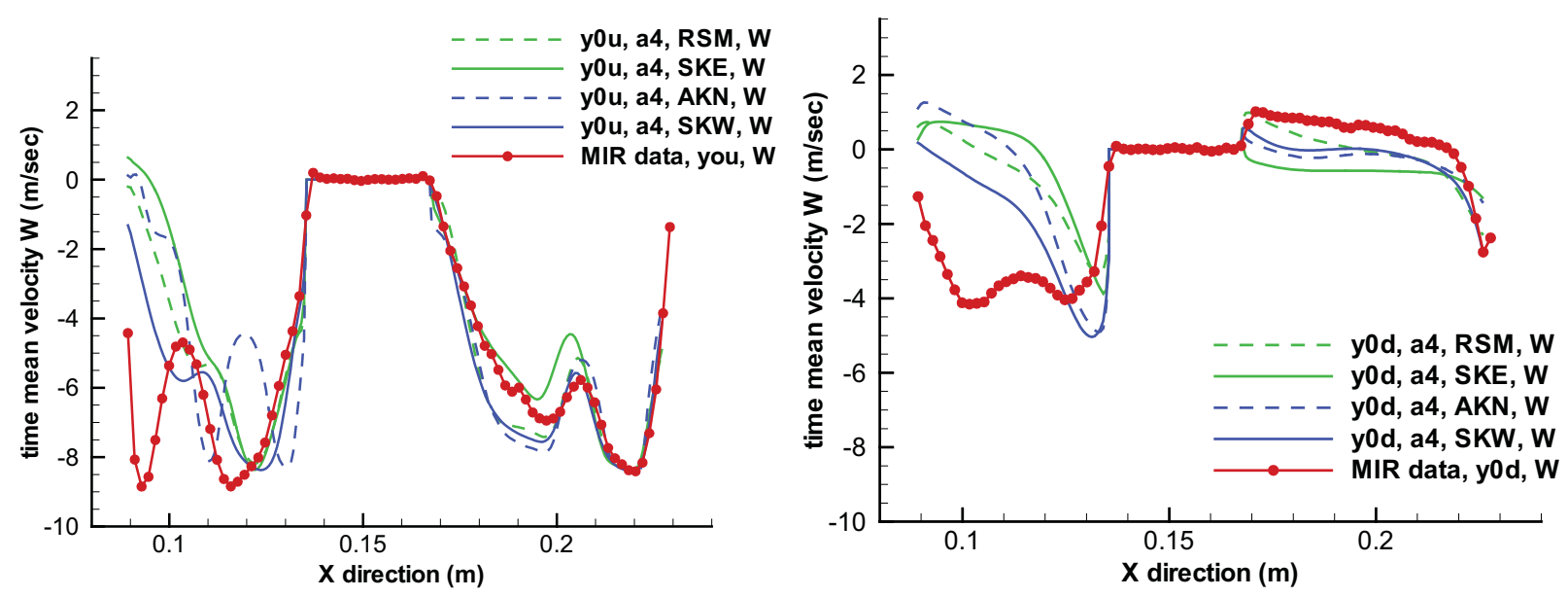

Figure 2-10. Time mean velocity $W$ at y0u and y0d.

It is insightful to compare contours of time mean vertical velocity $W$ for the four cases to obtain a better perspective of how well the flow is predicted. Figure 2-11 illustrates contours for $W$ at $\mathrm{Z}=-0.07 \mathrm{~m}$. Signatures of the four jets are visible in the plots. As can be seen, the RSM and SKE cases show similar asymmetric contours with Jet 1 skewed to the side; the AKN shows a skewing in the opposite sense, while the SKW shows a very symmetric solution. Apparently, the four turbulence models have "found" differing solutions. This may be because the flow is so unstable that there is no actual periodically stable solution. Also, the skewed results for Jet 1 explain the absence of a signature for Jet 1 in Figure 2-10 for the RSM and SKE models. For the AKN and SKW models, the Jet 1 signature is too shallow. 


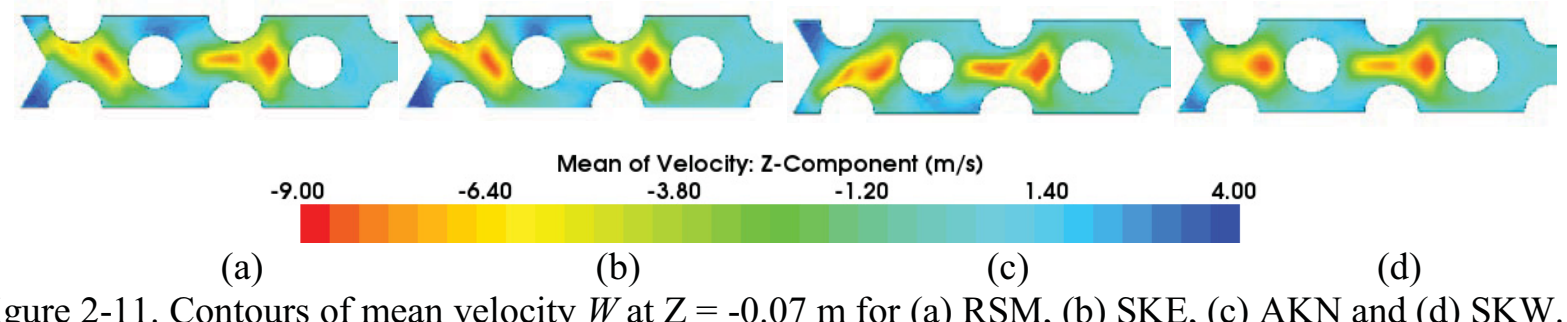

Figure 2-11. Contours of mean velocity $W$ at $Z=-0.07 \mathrm{~m}$ for (a) RSM, (b) SKE, (c) AKN and (d) SKW.

The MIR data show symmetry in the contours of $W$ at $Z=-0.07 \mathrm{~m}$ (Figure 2-12), indicating that the SKW model gets the more correct solution. The range of contour levels is the same for all graphics.

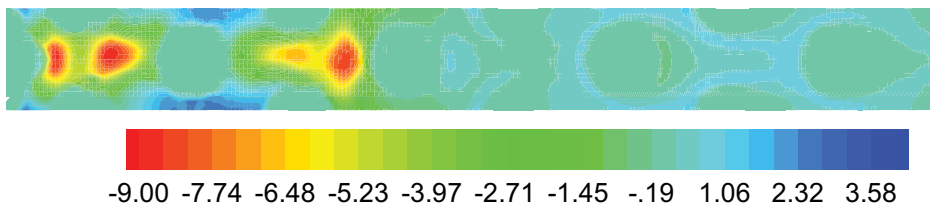

Figure 2-12. Contours of time mean velocity $W$ at $\mathrm{Z}=-0.07 \mathrm{~m}$ from the MIR data.

Figure 2-13 compares results for the four turbulence models with the MIR data for the stream-wise horizontal time mean velocity $U$ at profiles $x 3 \mathrm{u}, \mathrm{x} 3 \mathrm{~d}, \mathrm{x} 4 \mathrm{u}, \mathrm{x} 4 \mathrm{~d}, \mathrm{x} 6 \mathrm{u}$, and $\mathrm{x} 6 \mathrm{~d}$. At $\mathrm{x} 3 \mathrm{u}$, just upstream of Jet 3 , the flow is mostly vertical and the results differ significantly. At $x 3 \mathrm{~d}$, the flow has turned and become mostly horizontal. The spikes seen at $\mathrm{x} 3$ in the MIR data are not present in the calculations. The flow accelerates at $\mathrm{x} 4$ because of the presence of the two half posts. Again, the computed results vary widely, though the SKW results look best overall. By profile $x 6$, the effects of the jet inflow have reduced considerably and all of the results show correct trends. Overall, the calculations in the vicinity of the inlet jets show the poorest agreement. 

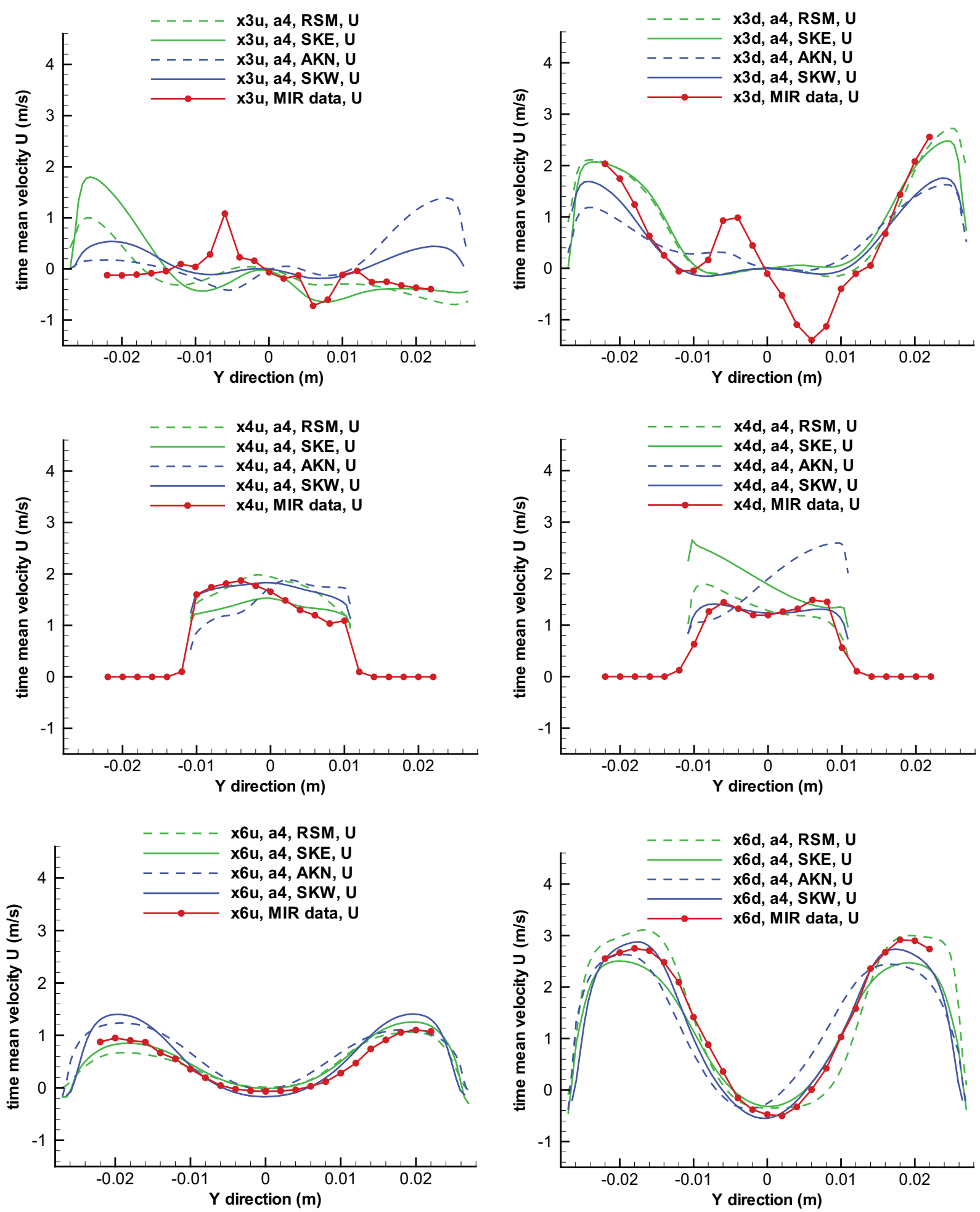

Figure 2-13. Comparison of time mean velocity $U$ results for the four cases with the MIR data. 


\subsubsection{Unstable Recirculation Zone}

Time evolution curves were accumulated during the computations to see if vortex shedding was occurring behind the cylinders. Figure 2-14 plots the stream-wise ensemble average velocity $\langle u\rangle$ at point $\mathrm{p} 7 \mathrm{~d}$ for the four turbulence models. While it is apparent that there are fluctuations occurring in the time traces for all four turbulence models, something else is also occurring for some of the calculations. The time trace for the SKW model (red) shows large excursions for $\langle u\rangle$ that seem to occur for random time intervals; this is particularly evident for the time interval of from about 3.8 to 4.8 seconds, where the average value for $\langle u\rangle$ would be about $2.75 \mathrm{~m} / \mathrm{sec}$, but is quite different before and after this interval. The time trace for the AKN model exhibits some random variations, but not nearly as dramatic as for the SKW model. The time traces for the other two models do not show such dramatic behavior.
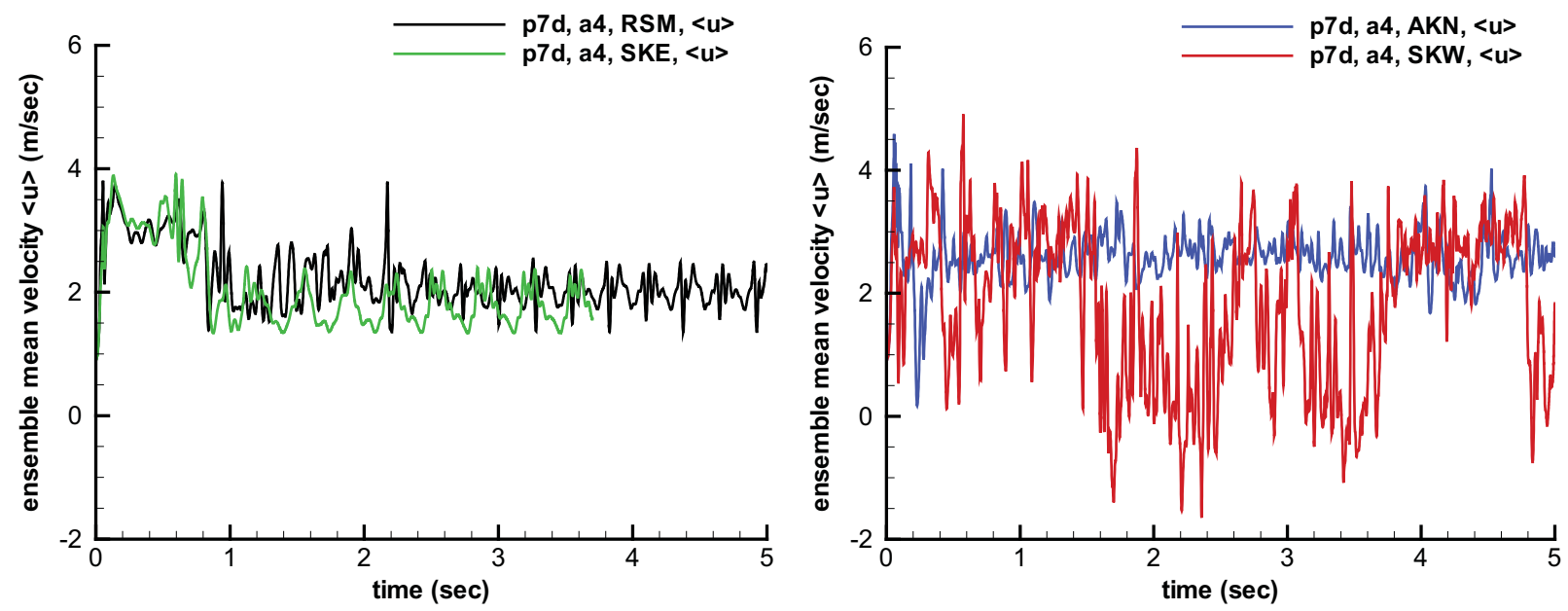

Figure 2-14. Time trace of ensemble average velocity $\langle u\rangle$ at point $\mathrm{p} 7 \mathrm{~d}$ for the four turbulence models.

Additional investigations were made to see if the random behavior occurs at other locations and for the MIR data. Figure 2-15 plots time evolution curves for the computed ensemble mean velocity $\langle w\rangle$ at points p1u and $\mathrm{p} 2 \mathrm{u}$ for the AKN and SKW models. Points $\mathrm{p} 1 \mathrm{u}$ and $\mathrm{p} 2 \mathrm{u}$ are located at the centers of Jets 2 and 3, respectively, at $\mathrm{Z}=-0.07 \mathrm{~m}$. The traces for the AKN and SKW models exhibit dramatic variations that again appear to be random in time.
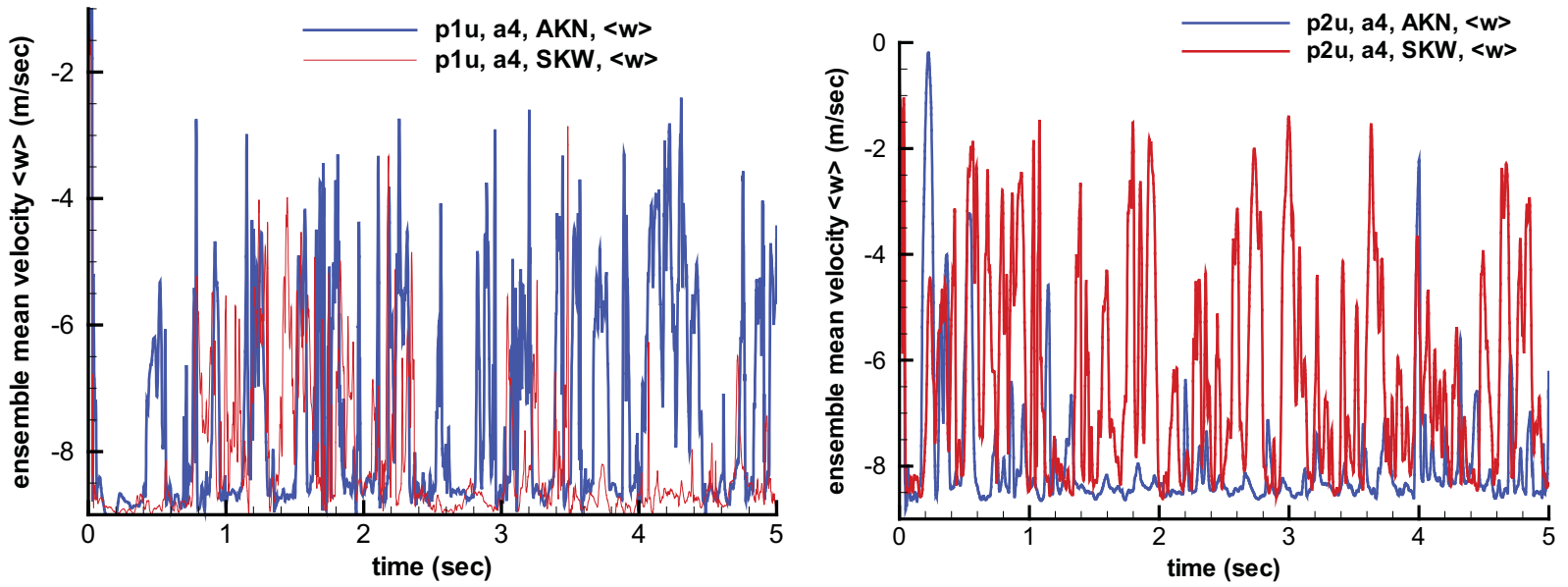

Figure 2-15. Time traces at points $\mathrm{p} 1 \mathrm{u}$ and $\mathrm{p} 2 \mathrm{u}$ for $\langle w\rangle$ for the AKN and SKW models. 
Time traces of MIR data were obtained to compare with the computations. Figure 2-16 plots the MIR data at points $\mathrm{p} 7 \mathrm{~d}, \mathrm{p} 1 \mathrm{u}$, and $\mathrm{p} 2 \mathrm{u}$, which compare to Figures 2-14 and 2-15. While the data are for a much longer time scale, the range of variation in the amplitudes for these traces are comparable to the traces for the SKW at p7d and for the AKN and SKW models at p1 $u$ and $p 2 u$. In fact, the variations in the MIR data cover a somewhat larger range than do the calculations. This provides clear evidence that dramatic excursions in the velocity are present, in accord with computations using the AKN and SKW models.
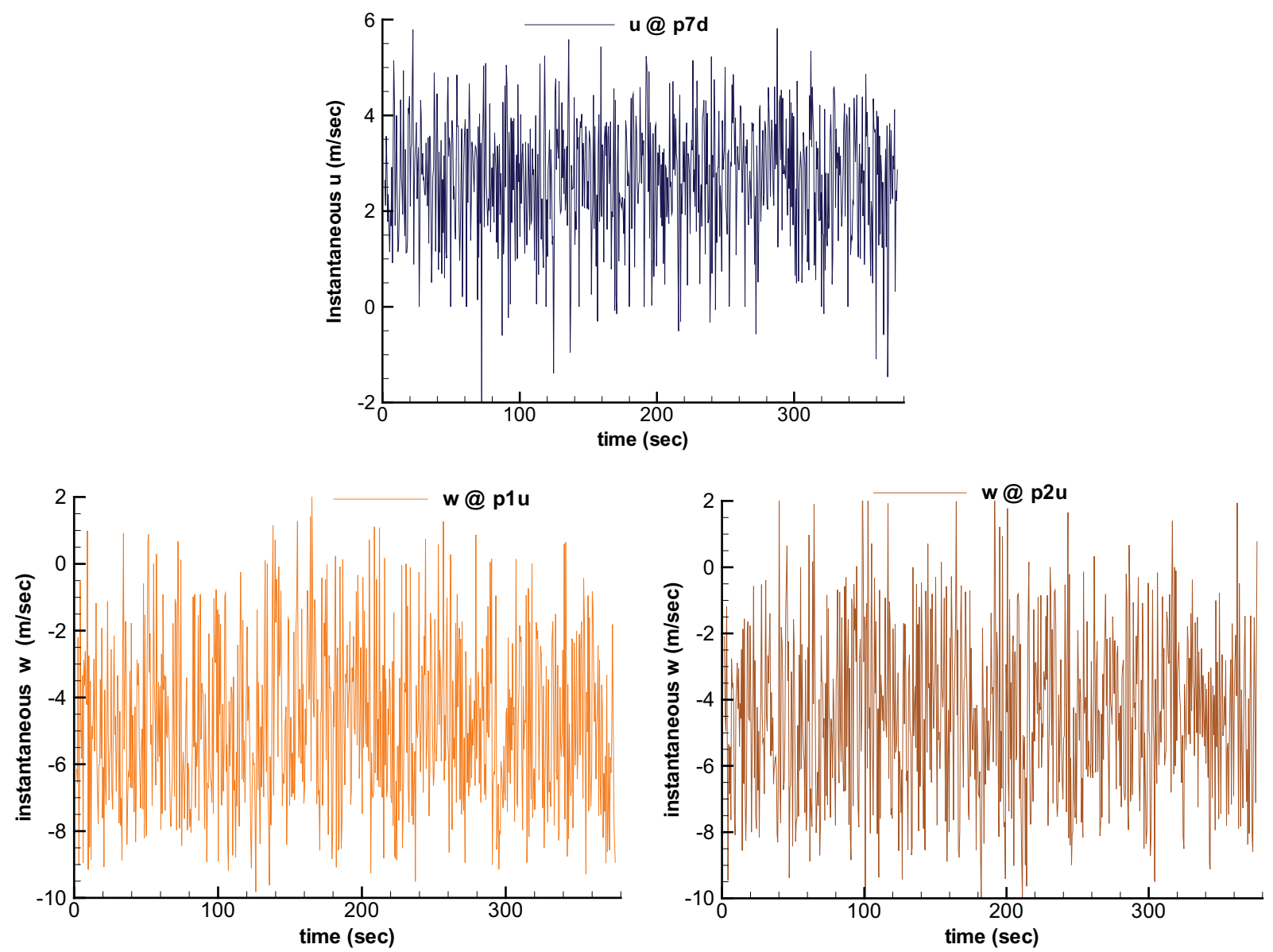

Figure 2-16. Time traces at points $\mathrm{p} 7 \mathrm{~d}, \mathrm{p} 1 \mathrm{u}$ and $\mathrm{p} 2 \mathrm{u}$ from the MIR data.

Figure 2-14 suggests that the four turbulence models produce different levels of turbulent viscosity because of the varying levels of damping of the time traces. In fact, when contour levels of the ratio of turbulent to molecular viscosity are plotted for the end times of the computations at $\mathrm{Z}=-.07 \mathrm{~m}$, Figure 2-17, it is seen that the RSM and SKE, which both show well-behaved periodicity in Figure 2-14, have similar relatively high levels, while the AKN has lower levels and the SKW has the lowest levels.

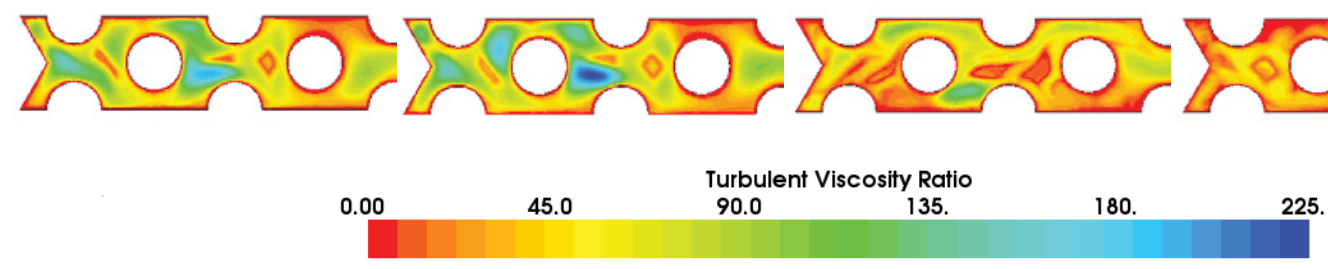

(a)

(b)

(c)

(d)

Figure 2-17. Turbulent to molecular viscosity ratio for the (a) RSM, (b) SKE, (c) AKN and (d) SKW. 
Additional investigations have shown that an unstable recirculation zone is present below Jets 1 and 2 (Johnson et al. 2010). Figure 2-18 illustrates streamlines calculated at specific points in time for the SKW model. As can be seen, the recirculation zone below the first two jets changes dramatically in time. The flow from the two jets is seen to detach from the left wall flowing outwards and then back around in a recirculating flow which then impinges upwards on the jets flowing downwards. This mutual impingement is apparently what causes the recirculation zone to change in time because of its instability. Figure 2-19 presents photographs from a flow visualization study performed in the MIR facility wherein air was injected into Jet 1 while fluid flowed into all four inlet ports. The photos are separated by a few tenths of a second in time. It can clearly be seen that the recirculation zone in the lower left corner changes in time, corroborating the streamline plots from the computations and providing evidence that the recirculation zone is changing dramatically in time.
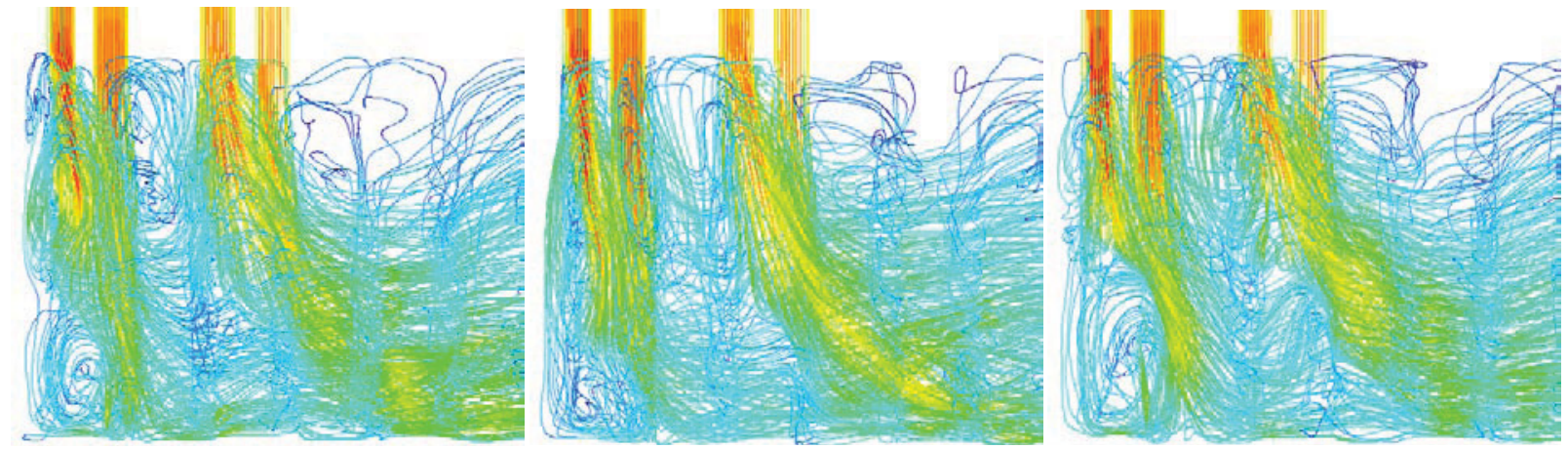

Figure 2-18. Streamline plots for the SKW case at 3.6, 3.9 and 4.2 seconds, respectively.
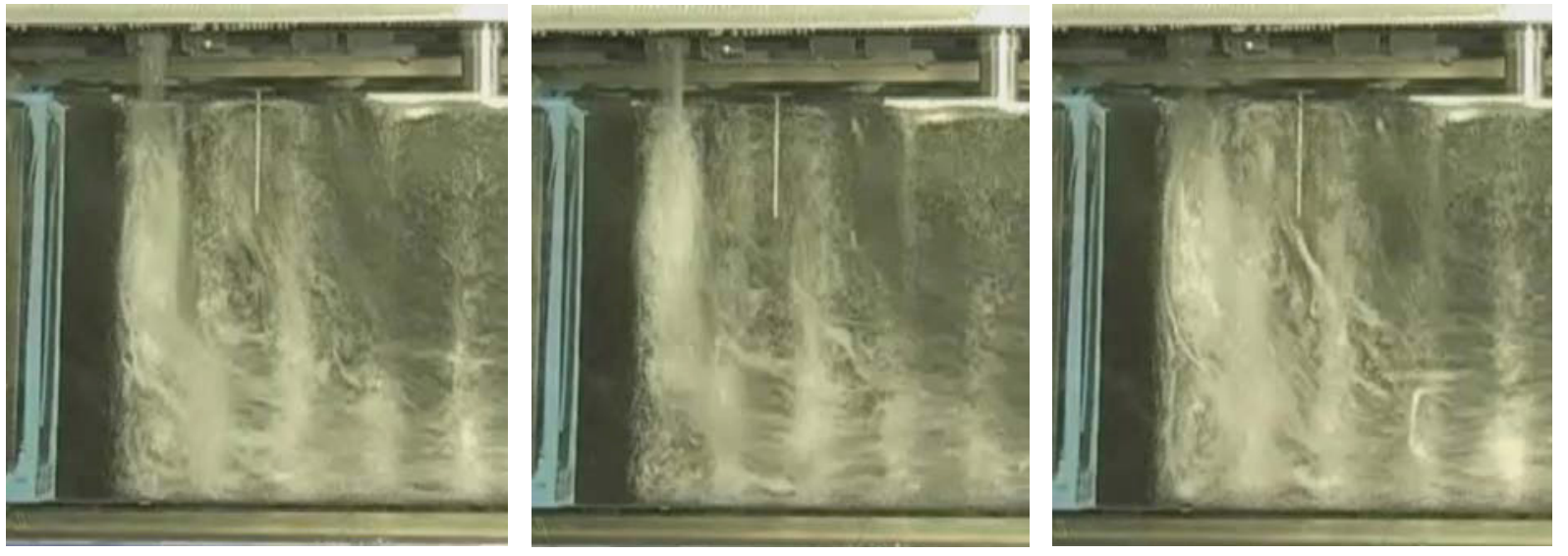

Figure 2-19. Flow visualization photos of the flow at different times.

In comparing the results for the four turbulence models, it is interesting to observe that the three with the highest turbulent viscosity levels have found inappropriate solutions for the first two jets. It may well be the case that the turbulence level in the scaled model is not very high, even though the inlet jets have a Reynolds number of 12,400 , well in the turbulent range. It is the low viscosity SKW model that shows the most appropriate behavior.

As shown from both computational results and the MIR data, as well as from flow visualization, the four-jet configuration produces an unstable flow situation wherein a recirculation zone changes size on an apparently random and relatively long time scale. Judging by the time traces plotted in Figure 2-16, if there is a long-time average of the flow that is unchanging, it would be over such a long time, probably dozens of seconds, that it would be entirely impractical to perform a calculation for validation purposes. 
For the present relatively coarse grid, the calculation of five seconds of flow time takes about 17 days using over 100 parallel processors. Hence, the presence of the unstable recirculation zone makes the present geometry undesirable for a validation data set. That is, the randomly varying, long-time-scale recirculation zone is an undesirable feature for a validation data set, not only because of the impracticability of computing it, but also because of its random nature. Rather, it seems much more appropriate to validate a CFD model with a problem close to that of the lower plenum flow, using a data set that does not include such a feature; one can then compute the flow with the undesirable feature with some confidence in the model because of the validation for the nearby problem.

\subsection{Results for Three-Jet Configuration}

It was decided to investigate the flow field for the case of shutting off Jet 1, inasmuch as it impinges on the recirculation zone and may be the cause of its random instability. Calculations were made using the same four turbulence models employed above for the four jet calculations and using the same best inlet conditions on grid a4 for the same time step; the first jet inlet surface is changed to be a wall. Figure 2-20 provides time traces for the calculations for points $\mathrm{p} 1 \mathrm{u}$ and $\mathrm{p} 2 \mathrm{u}$ with Jet 1 shut off; compare to Figure 2-15. Calculations of the time traces for all four models now show very low or no oscillations, after an initial transient period that results from using stagnant initial conditions, indicating that the randomly unstable recirculation zone has been stabilized (Johnson and McIlroy 2010; Johnson 2010). This is further confirmed by examining streamline plots of the flow at different times. Figure 2-21 shows 3-D streamline plots of the three-jet flow at three times for the SKW model. The plots show a large recirculation zone at the left boundary between the upper and lower extents of the model that appears to be unchanging for the three different times (Johnson et al. 2010). The recirculation zone is apparently now stable because there is no jet impinging on the top of the recirculation zone that would cause the detachment point to move up and down as is the case for the four-jet case of Figures 2-18 and 2-19.
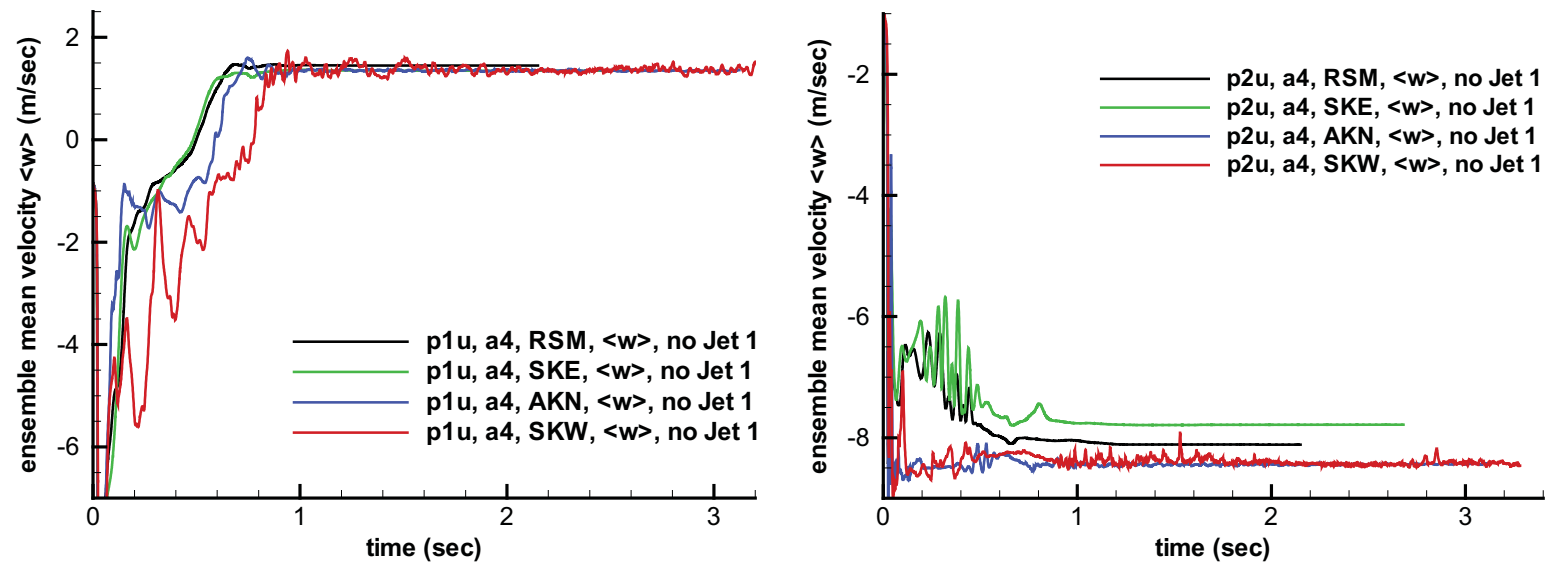

Figure 2-20. Time traces for points $\mathrm{p} 1 \mathrm{u}$ and $\mathrm{p} 2 \mathrm{u}$ for calculations for four models for the three-jet case. 

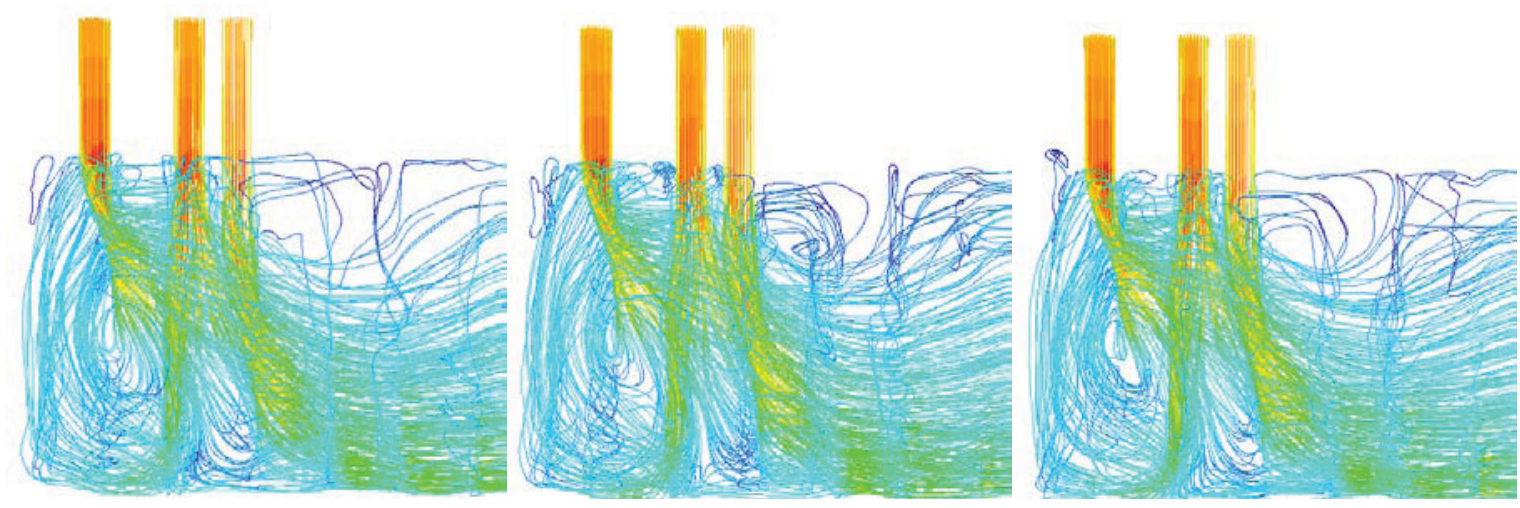

Figure 2-21. Streamline plots at 2.7, 3.0, and 3.28 seconds for the three-jet case for the SKW model.

Figure 2-22 plots the horizontal ensemble-averaged velocity $<u>$ for the three-jet case for the four turbulence models at point $\mathrm{p} 5 \mathrm{~d}$ (Johnson and McIlroy 2010). This point is rather far from the inlet jets where the flow is nearly horizontal (see Figure 2-8) and should be showing vortex shedding. The time traces for the RSM and SKE models, however, show the velocity reaching a steady state without vortex shedding after the initial transient. The trace for the AKN model show some regular fluctuations, but with low amplitude. It is not clear what kind of behavior this represents. Because the amplitude is so low, it could be related to pressure fluctuations but without actual vortex-shedding. Finally, the results for the SKW model indicate vortex-shedding with fairly large amplitude variations. It is shown in Figure 2-17 above that the four turbulence models used show increasingly greater levels of turbulent viscosity from the SKW to the AKN to the SKE to the RSM models for the same flow. This helps explain the range of fluctuations in the results in Figure 2-22.

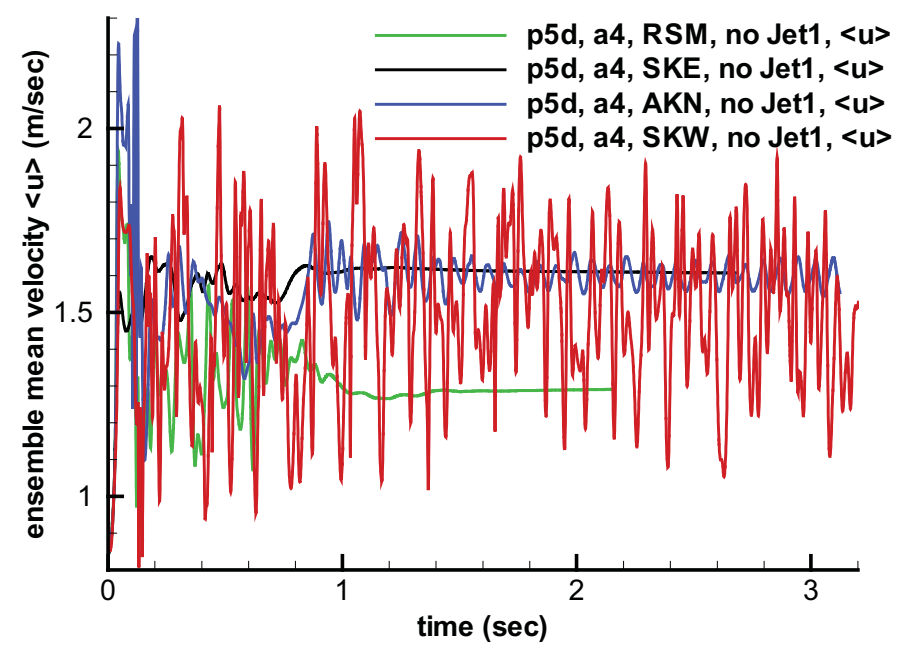

Figure 2-22. Trace of $\langle u\rangle$ at $\mathrm{p} 5 \mathrm{~d}$ for the three-jet configuration for the four turbulence models.

Profile predictions are now presented for the three-jet configuration. These profiles are for locations given by Figure 2-8 above. The results are long-time averaged, though the initial transients (see Figure 2-20) are not included in the averaging. They are compared to the MIR data for the four-jet configuration because three-jet data have not yet been taken. As such, these computations represent blind predictions. 
Figure 2-23 provides blind predictions for the three-jet configuration for time mean vertical velocity $W$ at y0u and y0d compared to the four-jet MIR data. Clearly, the calculations for the four turbulence models are closer to each other than for the four-jet calculations (see Figure 2-10). The signatures of the three remaining jets at y0 have changed significantly, except for Jet 4 for y0u. In addition to Jet 1 being gone, Jet 2 at y0u has been pushed downstream and up against a full cylinder located in the middle of the flow. At y0d, both the first and second jets are missing, though Jet 3 shows a strong signature, unlike the four-jet data. The signature for Jet 4 is seen to be stronger than for the four-jet data. Figure 2-24 plots contours of $W$ for a horizontal plane at $\mathrm{Z}=-07 \mathrm{~m}$ for the four turbulence models. These results are all quite similar to each other and show that Jet 2 is indeed pushed up against and partially wrapped around the first full cylinder as indicated in Figure 2-23. The signatures for Jets 3 and 4 are similar to those for the four-jet calculations (see Figure 2-11), though the three-jet results show rather better symmetry.
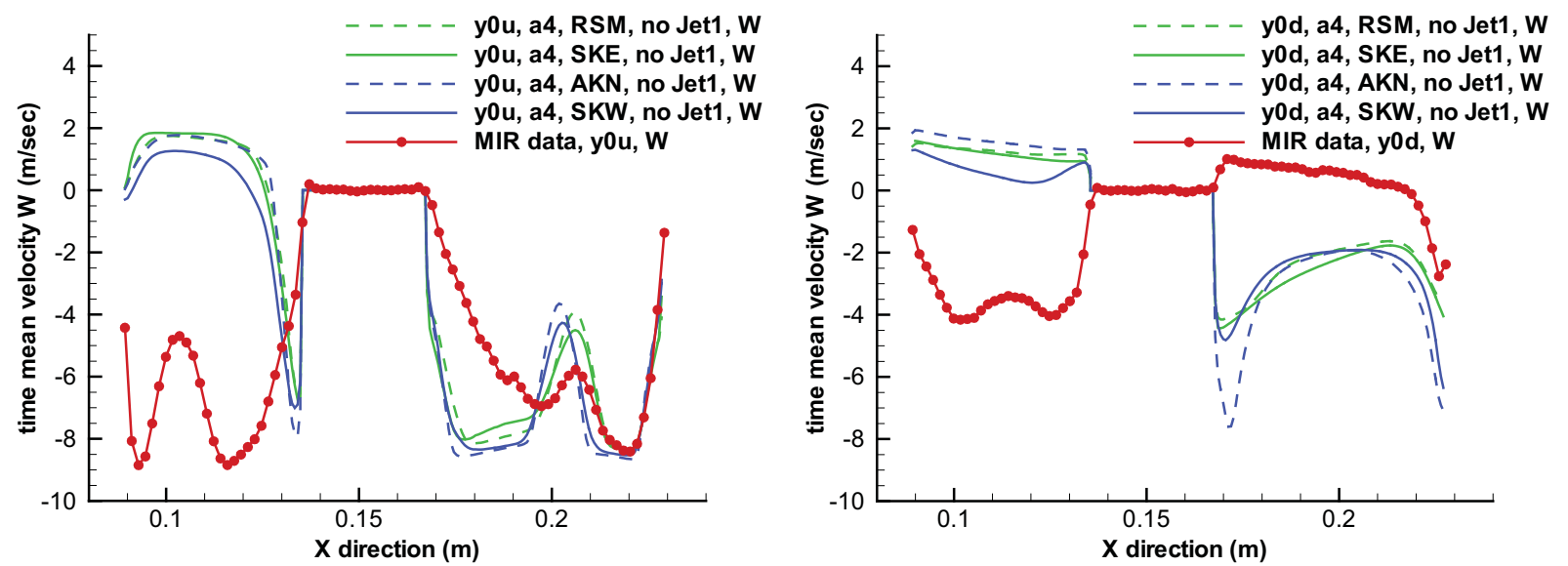

Figure 2-23. Time mean profiles for $W$ for stream-wise locations y0u and y0d for the three-jet results compared to the four-jet MIR data.

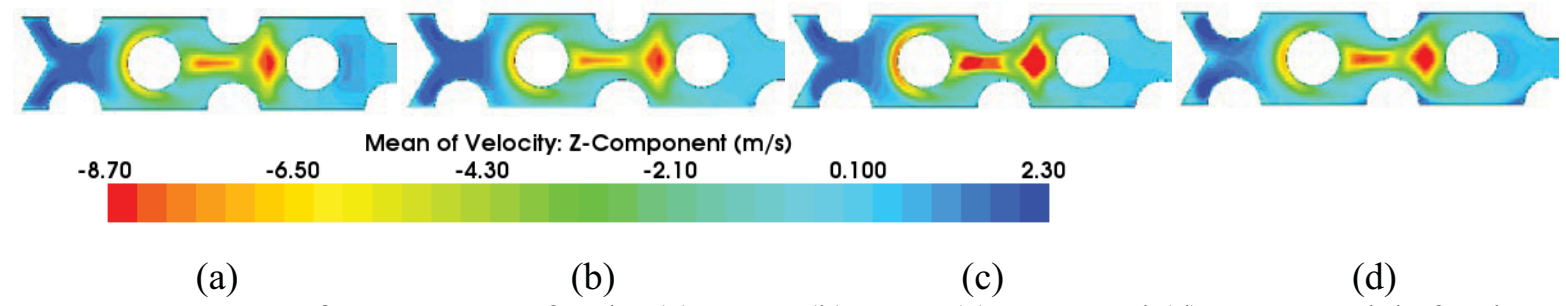

Figure 2-24. Contours of $W$ at $-0.07 \mathrm{~m}$ for the (a) RSM, (b) SKE, (c) AKN and (d) SKW models for the three-jet configuration.

Figure 2-25 plots transverse profiles for time mean vertical velocity $W$ at locations $x 1 \mathrm{u}$ and $\mathrm{x} 1 \mathrm{~d}$. The velocity is seen to be positive for both locations in contrast to the four-jet data. This is in accord with the streamline plots of Figure 2-21, which shows up-flow at elevations $Z=-0.07 \mathrm{~m}$ (or one-third the way from the top to the bottom) and $Z=-0.15 \mathrm{~m}$ (or two-thirds of the way down). 

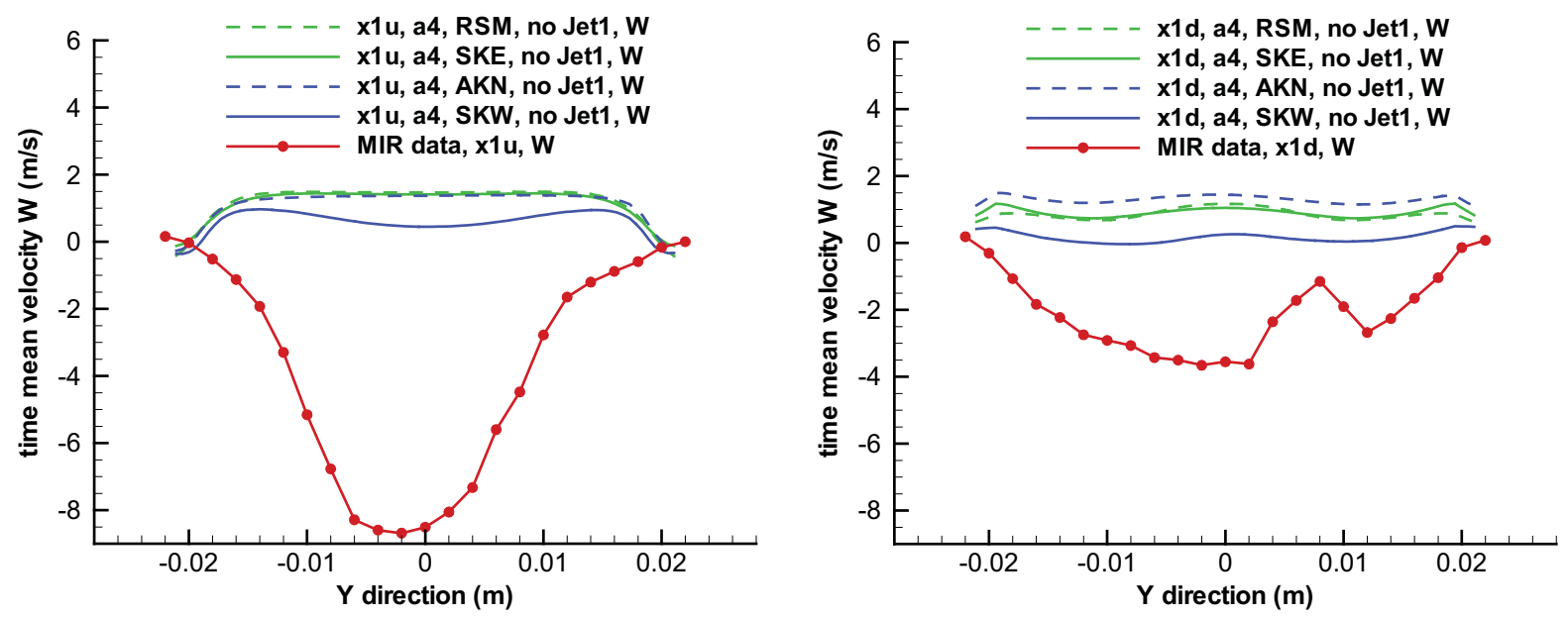

Figure 2-25. Profiles for $W$ for $\mathrm{x} 1 \mathrm{u}$ and $\mathrm{x} 1 \mathrm{~d}$ for the three-jet results compared to the four-jet MIR data.

Figure 2-26 plots the horizontal stream-wise time mean velocity $U$ along profiles $x 1 \mathrm{u}$ and $\mathrm{x} 1 \mathrm{~d}$. The velocity is positive at $\mathrm{x} 1 \mathrm{u}$ and negative at $\mathrm{x} 1 \mathrm{~d}$, which is also in accord with the recirculation zone seen in Figure 2-21. At $x 1 \mathrm{u}$, the SKE model shows a slightly different trend than the others with a maximum in the center. The trends are the same for $x 1 d$, though the SKW results are somewhat lower. Figure 2-27 plots results for $U$ at $x 2 \mathrm{u}$ and $\mathrm{x} 2 \mathrm{~d}$. The three-jet results are the reverse of the four-jet data. The velocity is positive at $\mathrm{x} 2 \mathrm{u}$ and negative at $\mathrm{x} 2 \mathrm{~d}$, which again matches the recirculation zone in Figure 2-21.

Predictions for $U$ at $\times 3 \mathrm{u}$ and $\mathrm{x} 3 \mathrm{~d}$ shown in Figure 2-28 are also the reverse of what the four-jet data show. Calculations at $\mathrm{x} 4 \mathrm{u}$ shown in Figure 2-29 are all symmetric in contrast to the four-jet data. The predictions for $\mathrm{x} 4 \mathrm{~d}$ are fairly close to the four-jet data; the SKW predictions are closest. Figure 2-30 illustrates results for $U$ at $\times 5 \mathrm{u}$ and $\mathrm{x} 5 \mathrm{~d}$. At $\times 5 \mathrm{u}, U$ is seen to be predicted to be either small or negative for all but the SKW model. At $x 5 \mathrm{~d}$, and at $\mathrm{x} 6 \mathrm{u}$ and $\mathrm{x} 6 \mathrm{~d}$, Figure 2-31 shows the flow to be strongly horizontal and close to the four-jet data, indicating a decrease in the influence of the inlet jets.
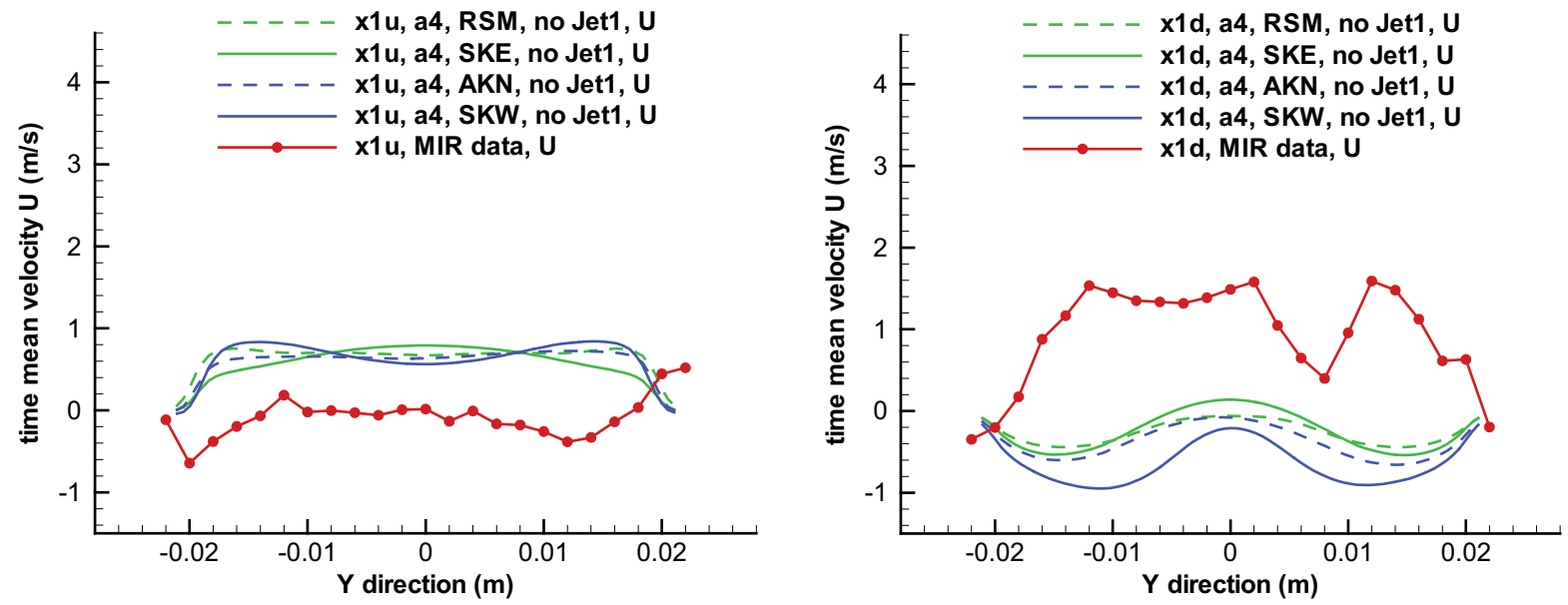

Figure 2-26. Time mean profiles for $U$ for transverse locations $\mathrm{x} 1 \mathrm{u}$ and $\mathrm{x} 1 \mathrm{~d}$ for the three-jet results compared to the four-jet MIR data. 

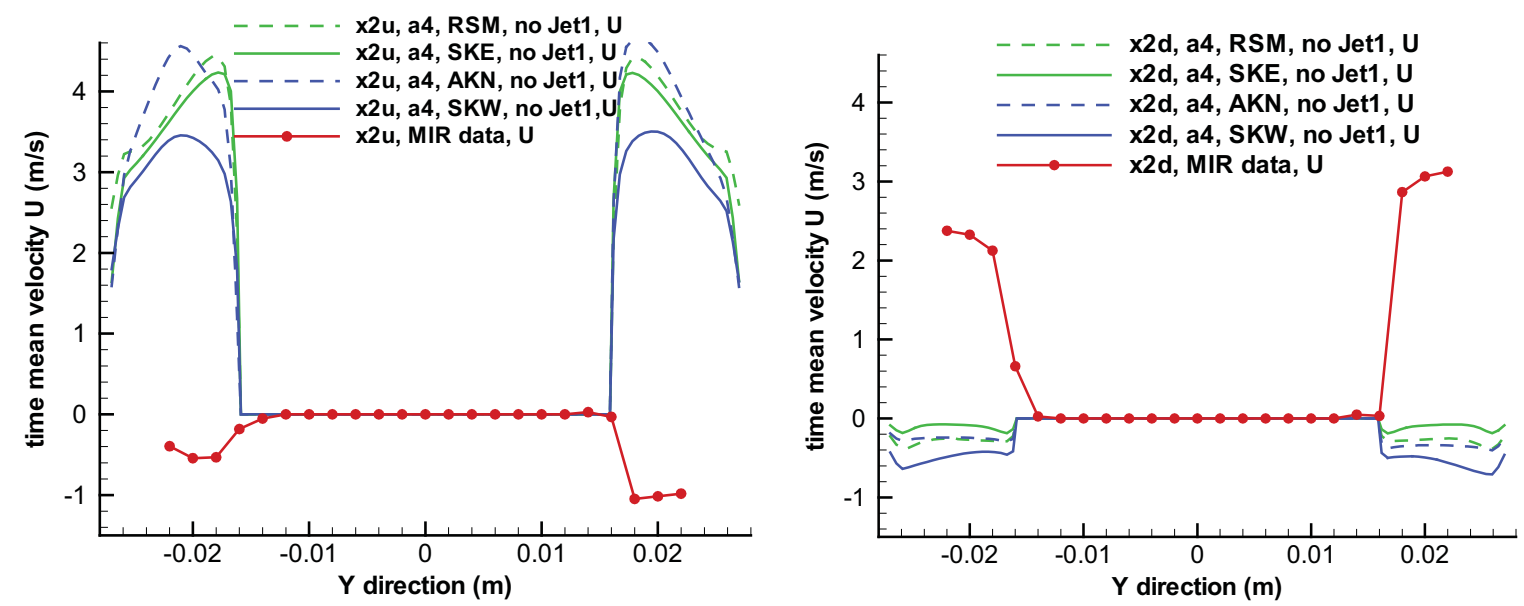

Figure 2-27. Time mean profiles for $U$ for transverse locations $\times 2 u$ and $\times 2 d$ for the three-jet results compared to the four-jet MIR data.
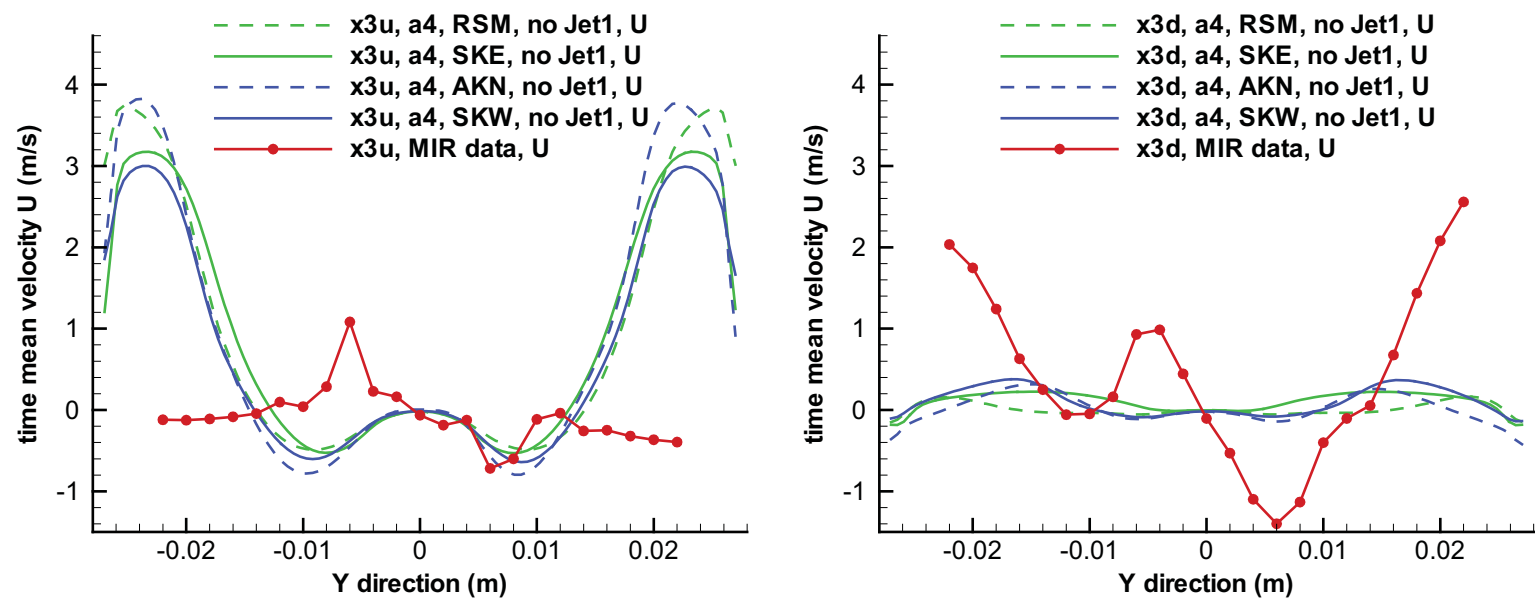

Figure 2-28. Time mean profiles for $U$ for transverse locations $\times 3 \mathrm{u}$ and $\mathrm{x} 3 \mathrm{~d}$ for the three-jet results compared to the four-jet MIR data.
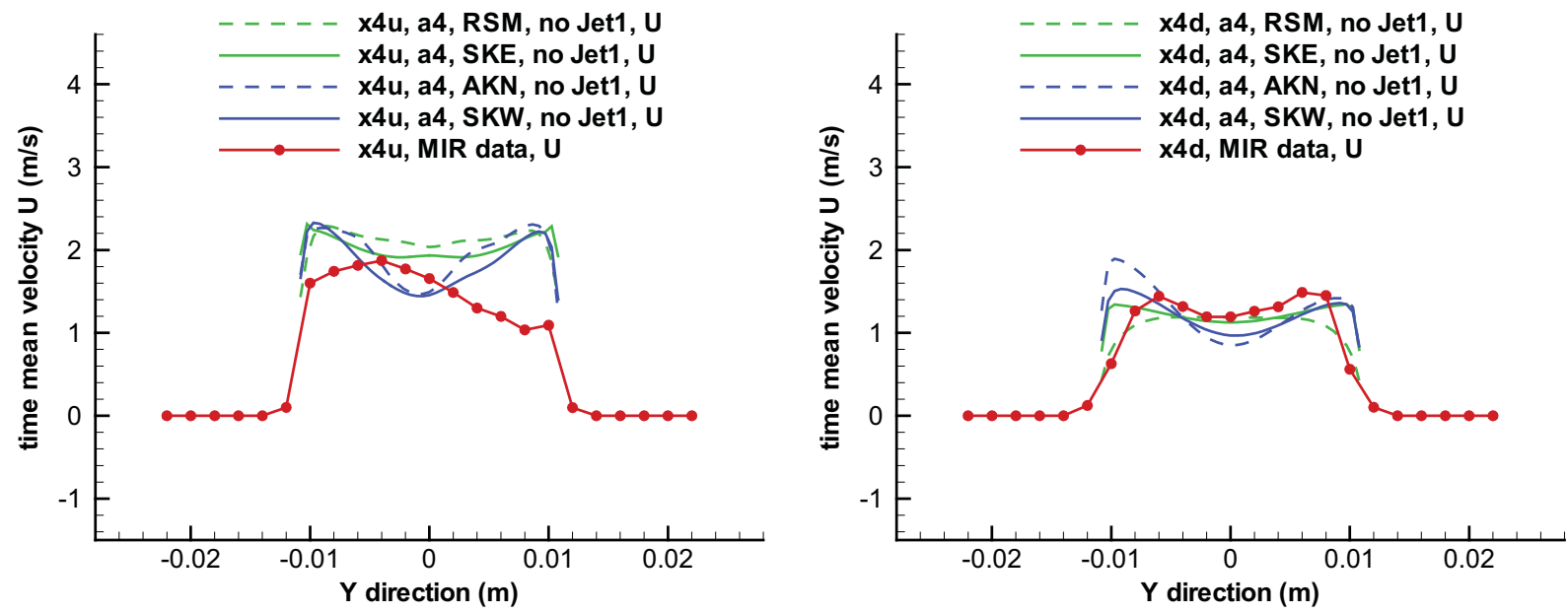

Figure 2-29. Time mean profiles for $U$ for transverse locations $\mathrm{x} 4 \mathrm{u}$ and $\mathrm{x} 4 \mathrm{~d}$ for the three-jet results compared to the four-jet MIR data. 

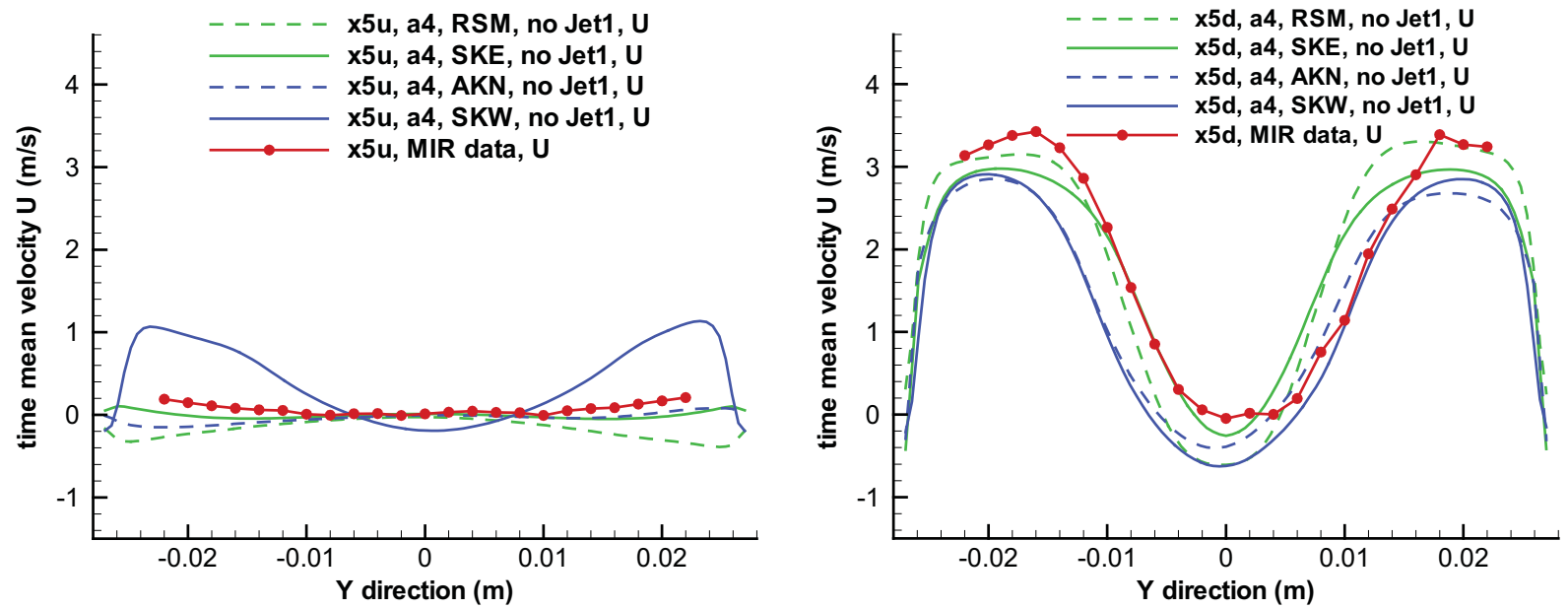

Figure 2-30. Time mean profiles for $U$ for transverse locations $x 5 \mathrm{u}$ and $\mathrm{x} 5 \mathrm{~d}$ for the three-jet results compared to the four-jet MIR data.
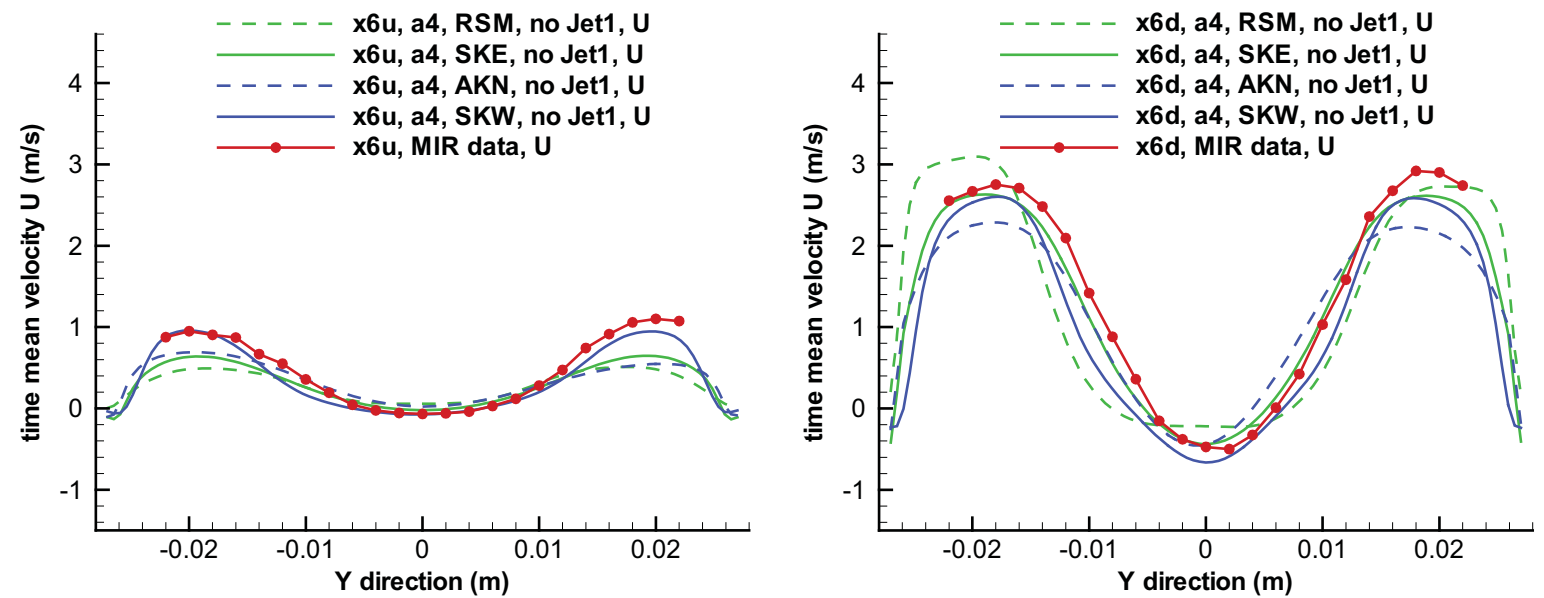

Figure 2-31. Time mean profiles for $U$ for transverse locations $x 6 \mathrm{u}$ and $\mathrm{x} 6 \mathrm{~d}$ for the three-jet results compared to the four-jet MIR data.

Two significant objections for the existing MIR data to be useful for validation purposes have been identified and discussed. These are first the discrepancy between the measurements of inlet mass flow between the calibrated rotameters and the detailed PIV data and second the presence of an unstable and randomly varying recirculation zone in the bottom corner of the model below the first two jets. The reasons that the presence of the unstable zone makes the data unsuitable are first that the instability appears to be random in time such that there may not be a periodically stable solution and second that the time scale of the change is about an order of magnitude longer than the time scale of shedding vortices (Johnson 2010), making it very impractical to compute because of the long simulation time that would be needed. It seems far more appropriate to validate a CFD model for the three-jet case and then apply it to the problem of the four-jet data to see if it can be calculated. It is recommended that the MIR data be retaken with improved accuracy for the inlet conditions and with Jet 1 turned off. 


\subsection{Summary and Recommendations for the Lower Plenum Standard Problem}

Experimental data were earlier taken in the INL's MIR test facility for a narrow scaled section of the lower plenum of a prismatic VHTR reactor with the objective of providing a CFD validation data set or standard problem. The present report provides a summary of CFD simulations performed to investigate the suitability of the data for validation purposes. Important findings include the observation that inlet data taken using PIV do not match the flow rates measured by calibrated rotameters. This discrepancy is a serious shortcoming of the MIR data and should be remedied. Present calculations were made using the rotameter data. Four turbulence models were used to simulate the MIR data. Time trace results from the SKW model indicate that an unstable recirculation zone present below Jets 1 and 2 causes significant perturbation to the flow field with an apparently random time scale. The large amplitude oscillations seen in the calculations for the SKW model are also seen in the MIR data; corroboration is also obtained from photographs taken for a flow visualization study. CFD calculations show large differences amongst the four turbulence models employed and the data. Calculations made with Jet 1 turned off indicate that the unstable zone has been stabilized and that CFD results for the four models are more closely matched. The calculations represent blind predictions for flow in the scaled model. It is recommended that the MIR data be retaken with improved accuracy for the inlet conditions and with Jet 1 turned off. Finally, it is clear from the above CFD investigation that CFD should be used in the design of new validation data sets. 


\section{BYPASS FLOW STANDARD PROBLEM}

\subsection{Introduction}

Significant uncertainty exists about the effects of bypass flow in a prismatic gas-cooled VHTR. Bypass flow occurs in the gaps between prismatic graphite blocks in the core. The gaps are present because of variations in their construction, imperfect installation and expansion and shrinkage from thermal heating and neutron fluence. Figure 3-1 provides an isometric illustration of bypass flow in the prismatic core. The blue arrows represent normal coolant flow in the appropriate coolant channels. Red arrows represent flow in the interstitial gaps between the blocks. It is not intended that flow occur in the gap regions between blocks. While there is also bypass flow that occurs in other regions, such as fuel rod channels, the focus of the present standard problem is on bypass flow between prismatic blocks. As shown in Figure 3-1, there may be lateral (horizontal) flow in the gap regions, depending on local pressure gradients and gap geometries.

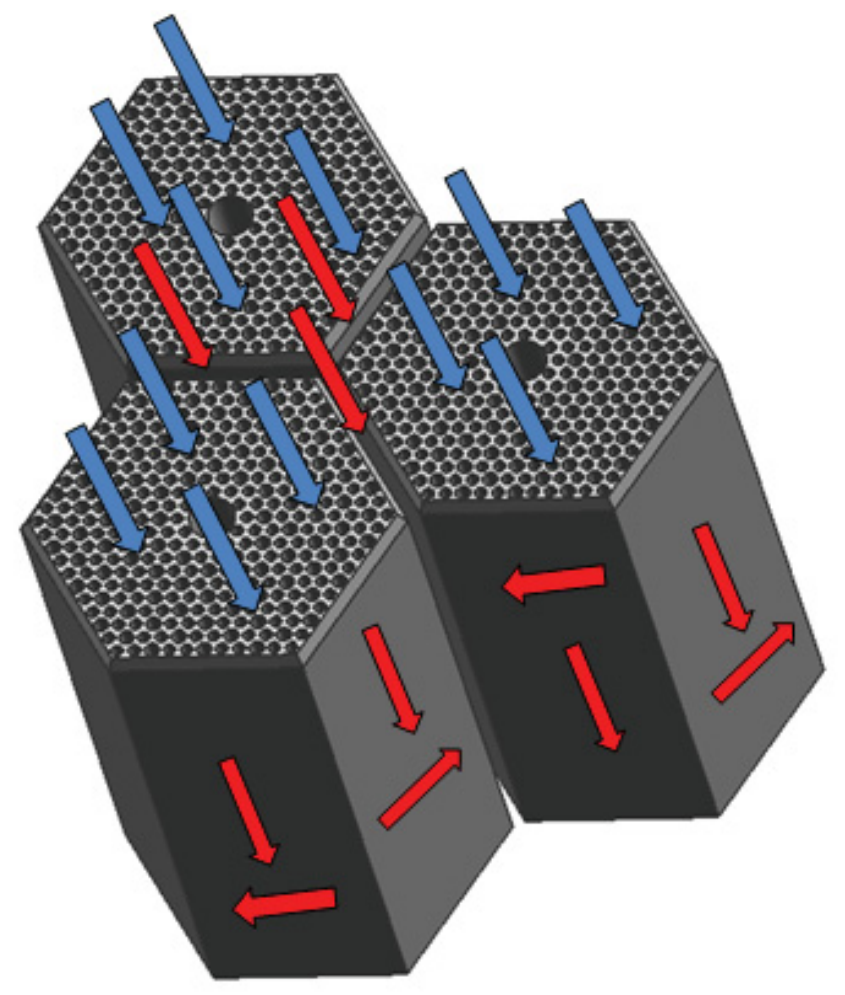

Figure 3-1 Schematic of bypass flow between prismatic blocks.

The percent of bypass flow that occurred in earlier gas-cooled reactors, such as the Fort St. Vrain Gas-Turbine Modular Helium Reactor (GT-MHR), was not known because of insufficient instrumentation, but was suspected to be as high as 15 to $20 \%$ of the total flow of coolant in the reactor. At this level, the effects of bypass flow should be better understood. With the advent and maturing of computational fluid dynamics, it should be possible to perform computations to estimate the level and effects of bypass flow in a prismatic reactor.

Several studies have been performed to investigate bypass flow. Researchers have used a variety of approaches to analyze the flow and heat transfer in a block VHTR. Simple approaches using networks and simplified unit cell methods have been employed (e. g. Takada et al. 2004; Nakano, Tuji, and Tazawa 2008). More advanced approaches have also been employed using conjugate flow and heat transfer 
available in commercial CFD codes. Tak, Kim, and Lee (2008) used a symmetric one-twelfth sector of a prismatic block. However, Tak, Kim, and Lee (2008) determined inlet flows from separate 1D code calculations. Recently, Sato, Johnson, and Shultz (2010) employed a similar one-twelfth sector analysis using commercial code FLUENT v. 6.3 .26 (see Appendix B) to investigate the effects of bypass flow for a $600 \mathrm{MWth}$ prismatic VHTR, based on GA GT-MHR, which originally was used as the reference design for the NGNP (MacDonald et al. 2003). In Sato, Johnson, and Schultz (2010), the flow rates were determined as part of the solution by using pressure boundary conditions for the inlet and outlet.

\subsection{Computational Bypass Flow Study}

Johnson and Sato (2010) provide bypass flow calculations for the 350 MWth Modular High Temperature Gas Reactor (MHTGR) (GA Technologies 1992), which is currently considered the reference prismatic VHTR design. The one-twelfth sector used by Johnson and Sato (2010) is shown in Figure 3-2. Each of the three side boundaries is a symmetry boundary. The one-twelfth sector extends through the entire core in the vertical direction with an upper reflector section, 10 fueled blocks and a lower reflector, making the model $10.704 \mathrm{~m}$ high. Inlet temperature for the cases investigated was set to $259^{\circ} \mathrm{C}$ as specified for the MHTGR (GA Technologies 1992). The flow was induced by specifying a stagnation pressure inlet and a static pressure outlet. The pressure difference specified was $29.9 \mathrm{kPa}$ for the no-gap case. However, the pressure is adjusted for the finite-gap cases to indicate that the bypass flow is stolen from the coolant channel flow. The heat generation is set to a constant in the fuel channels such that the overall core produces $350 \mathrm{MWth}$. Helium properties, assumed to be isobaric at $6.4 \mathrm{MPa}$, are obtained from the National Institute of Standards and Technology website ${ }^{\mathrm{b}}$. The graphite properties are based on H-451 graphite (General Atomics 1977). The fuel compact properties are taken from previous thermal hydraulic studies conducted by the INL (MacDonald et al. 2003). Results shown below are from Johnson and Sato (2010).

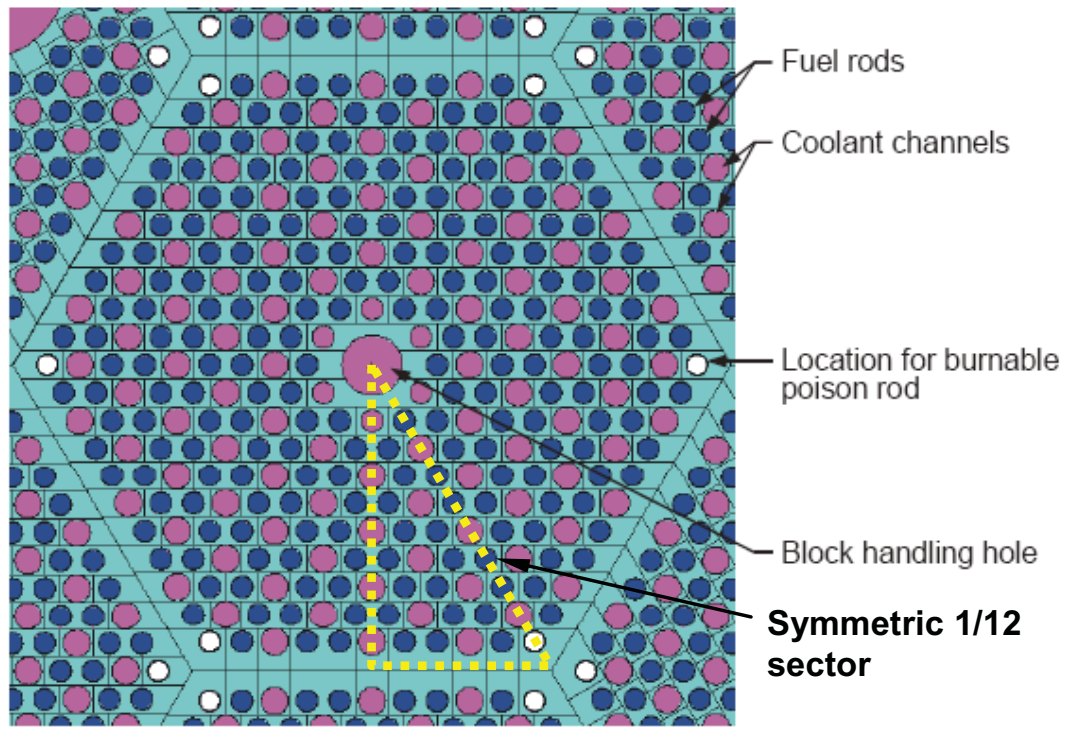

Figure 3-2. Plan view of a hexagonal block and one-twelfth symmetric sector.

b. http://webbook.nist.gov/chemistry/fluid/ 
Figure 3-3 shows temperature contours for three cases of increasing gap width from 0 to 3 to $5 \mathrm{~mm}$ at the plane that shows the highest fuel temperature, about $0.06 \mathrm{~m}$ above the lower edge of the fueled section. As can be seen, the maximum fuel temperature, which is near the block center, increases significantly as the gap width increases from 785 , to 808 , to $841^{\circ} \mathrm{C}$. It can also be seen that the graphite near the gap decreases significantly in temperature, causing a rather large lateral temperature gradient with increasing gap width. Additionally, the variation in coolant temperature at the outlet for a given block increases from 36 to 77 to $130^{\circ} \mathrm{C}$ for the three gap widths. This may have significance for structural integrity because of increased thermal stresses. It also has significance for flow in the lower plenum, with an increased temperature variation in the coolant entering the lower plenum. The inputs and results for the three cases shown are summarized in Table 3-1. As can be seen, the total mass flows for all three cases are approximately the same. Thus, the mass flow in the gap is stolen from the coolant channels.

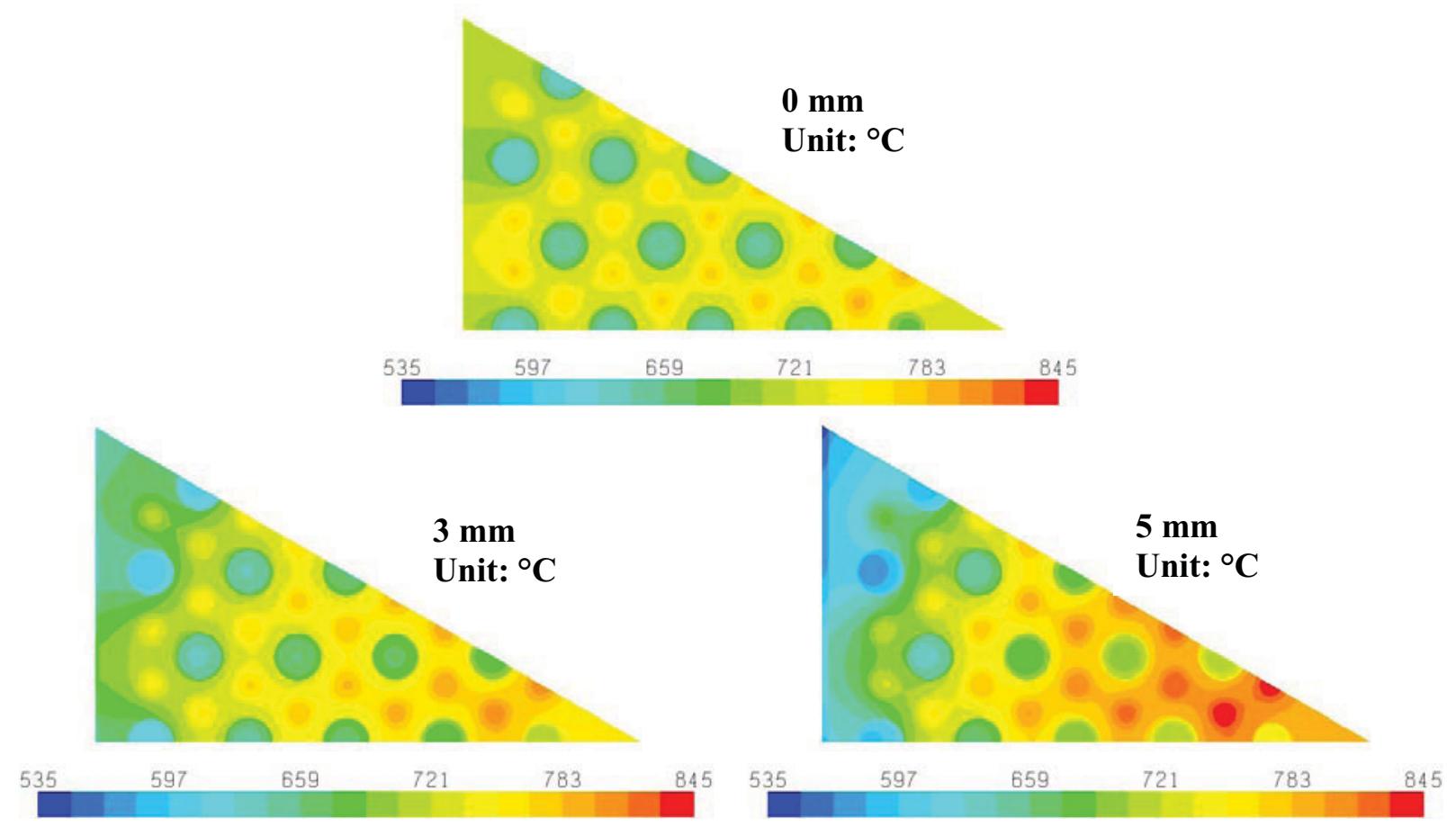

Figure 3-3. Temperature contours for the 0,3 , and $5 \mathrm{~mm}$ gap cases for constant mass flow rate.

Table 3-1. Inputs and results for the gap width study.

\begin{tabular}{|l|l|l|l|}
\hline Gap width mm & 0 & 3 & 5 \\
\hline Total mass flow $(\mathrm{kg} / \mathrm{s})$ & 0.210 & 0.211 & 0.211 \\
\hline Pressure drop $(\mathrm{Pa})$ & 29,900 & 27,782 & 25,024 \\
\hline \hline Gap flow fraction $(\%)$ & 0 & 4.27 & 9.87 \\
\hline Maximum fuel temp. $\left({ }^{\circ} \mathrm{C}\right)$ & 785 & 808 & 841 \\
\hline Gap outlet temp. $\left({ }^{\circ} \mathrm{C}\right)$ & - & 637 & 606 \\
\hline Maximum channel outlet temp. $\left({ }^{\circ} \mathrm{C}\right)$ & 685 & 704 & 734 \\
\hline Maximum helium temp. variation $\left({ }^{\circ} \mathrm{C}\right)$ & 36 & 77 & 130 \\
\hline Bulk outlet temp. $\left({ }^{\circ} \mathrm{C}\right)$ & 662 & 661 & 661 \\
\hline
\end{tabular}

In their studies, Sato, Johnson, and Schultz (2010) performed partial validations by comparing computed wall shear stress with published correlations for friction factor. They showed that calculations 
were in very good agreement for a particular turbulence model in FLUENT (standard $k \sim \varepsilon$ model with enhanced wall treatment). The same model and CFD mesh were used in Johnson and Sato (2010). However, the heat transfer was not validated. Neither were detailed velocity calculations. The purpose of the present development of a standard problem is to provide validation data for bypass flow, particularly in the interstitial regions between graphite blocks.

\subsection{Bypass Flow Standard Problem Geometry}

A scaled model has been developed for installation in the INL's MIR test facility. The scale is a factor of 2.016 greater than the actual geometry. The model is based on the MHTGR (GA Technologies 1992) prismatic reactor. Figure 3-4 gives a sketch of three adjacent prismatic blocks from a GA MHTGR. Because the focus of the standard problem is the gap flow, the model was chosen to center on the gap region between three blocks. The scaled model geometry is bounded by the thick red line in the figure. Only three standard coolant channels are included from each block in the model. The junction of the three gaps is the center of the scaled model.

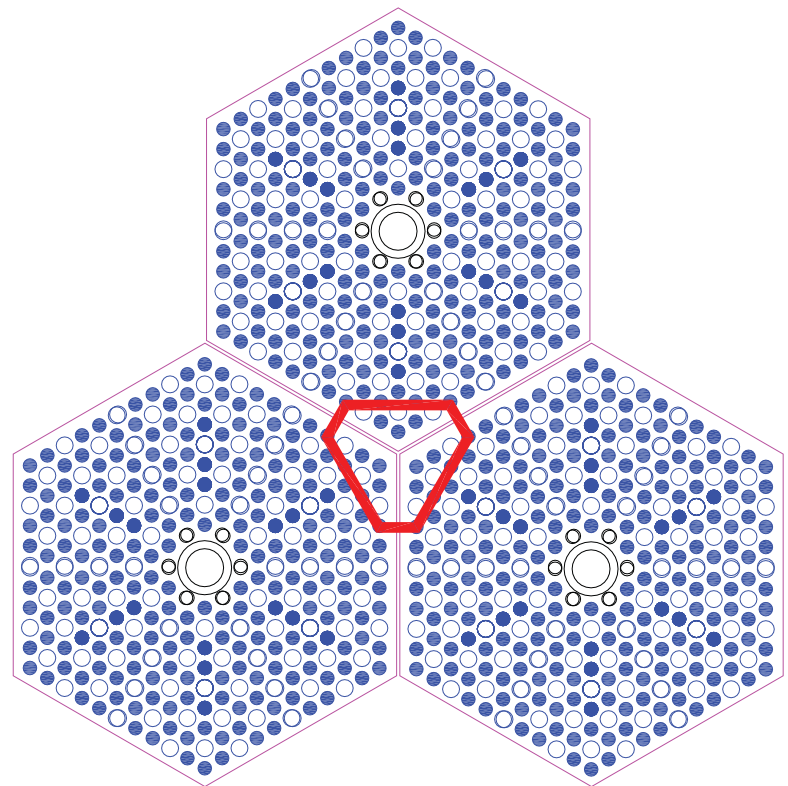

Figure 3-4 Three prismatic blocks from the MHTGR reactor and outline of test model geometry.

Figure 3-5 shows a close-up view of the source of the model geometry and a view that eliminates those things that are extraneous to the bypass model geometry. The lines parallel to the gap edges represent bevels machined in the graphite in the original MHTGR prismatic blocks. Three coolant channels are present for each block. Note that the flow in the bypass scaled model will be isothermal.

Figure 3-6 illustrates the primary components of the bypass flow model. These include the inlet annulus where the working liquid (mineral oil) enters and is then directed upwards, a hemisphere which redirects the flow downwards, an upper plenum, an upper fuel block, an adjustable horizontal gap, a lower fuel block, and two orifice plates that enclose screens in the channel and gap flow paths. The screens are intended to increase the pressure drop over the shorter lower fuel block to the same value as the upper fuel block. Also shown are the flow paths of the mineral oil along with the layout of the supported model as it will sit in the working section of the MIR test facility. The bypass model is designed to have adjustable gap widths. The vertical gaps visible in Figure 3-5 are adjustable from 2, to 6, to $10 \mathrm{~mm}$, representing 1,3 , and $5 \mathrm{~mm}$ in the actual block size. The horizontal gap can be set to widths of either 2 or $10 \mathrm{~mm}$. 

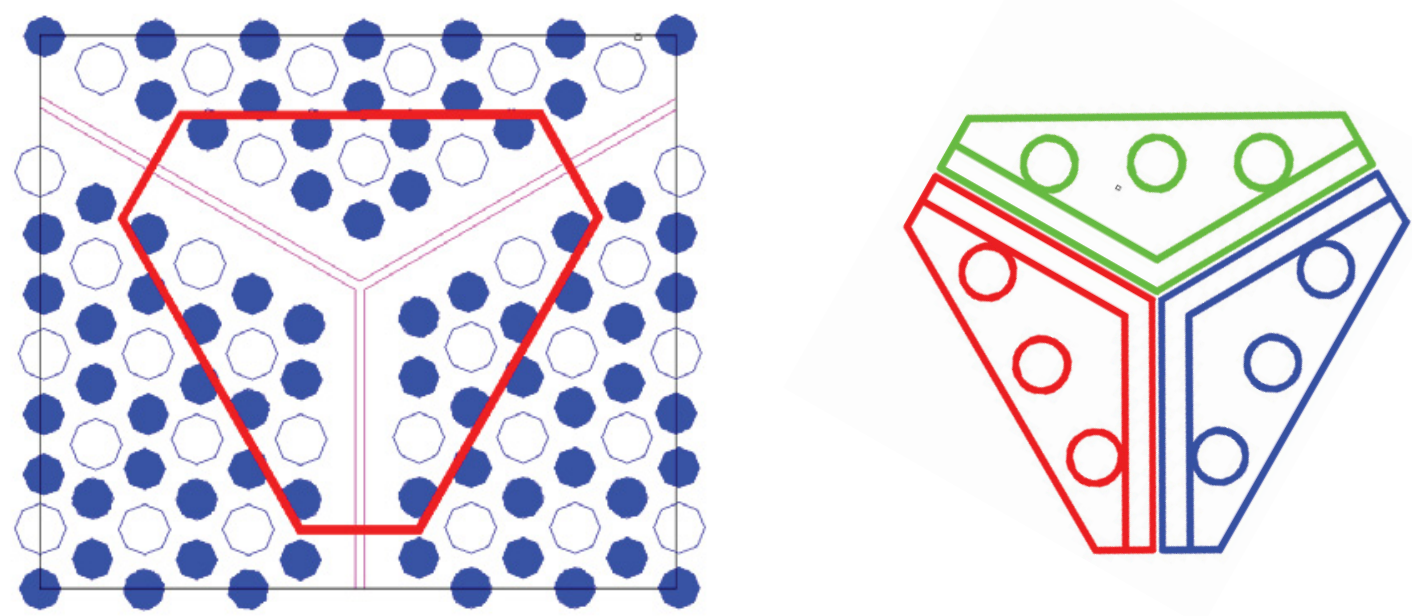

Figure 3-5. Close-up view of the origin of the bypass flow model and a cross-section view of the model.

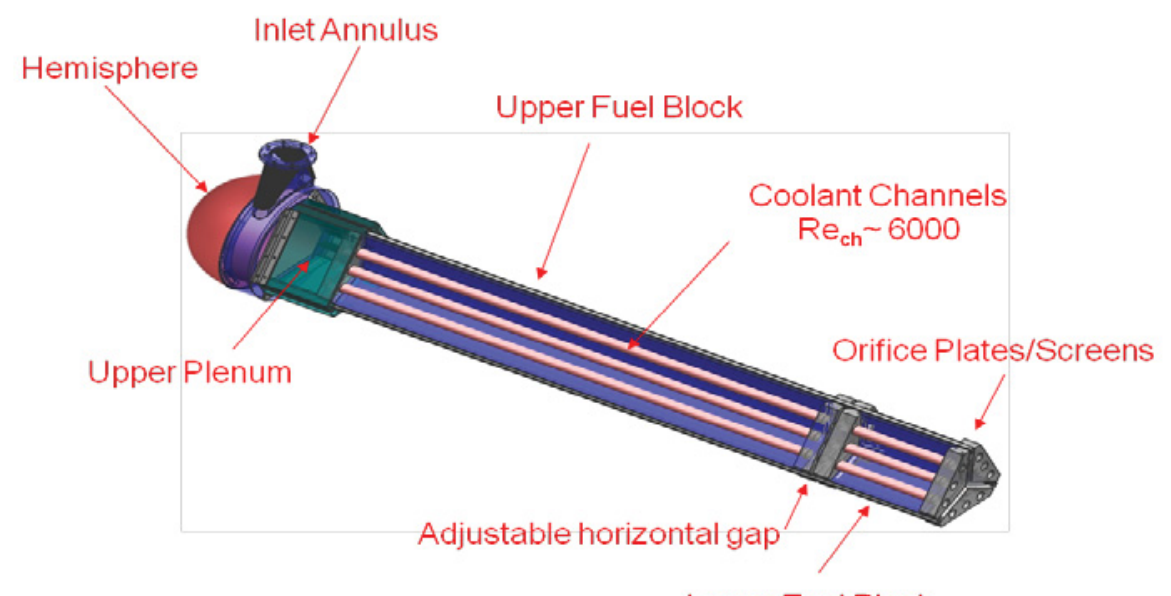

Lower Fuel Block

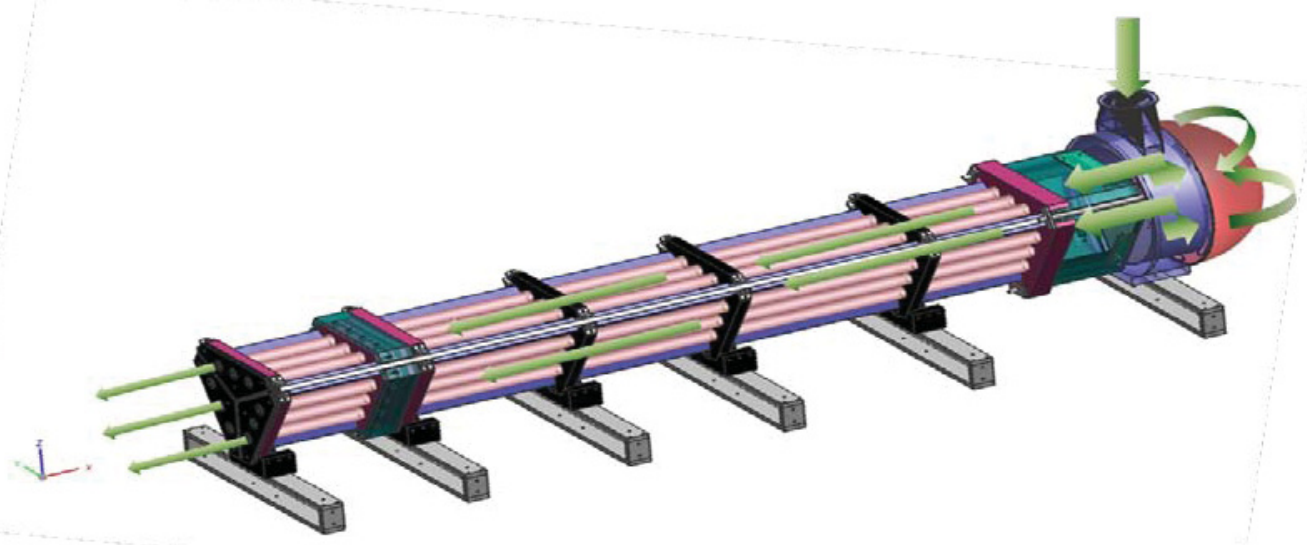

Figure 3-6. Schematics of the bypass model showing components and flow paths. 


\subsection{CFD Model}

A CFD model has been constructed and used to perform preliminary computations of flow in the MIR scaled model. Figure 3-7 provides a top view of the model. Because of symmetry, the CFD model includes only one-sixth of the actual model. Also shown in Figure 3-7 is the grid of the top surface of the CFD model; the grid was constructed using GAMBIT v. 2.4.6 (see Appendix B). This grid is extruded down through the model, except for the volume above the bevels in the blocks. The 1-1/2 coolant channels are shown. The bevel region can be seen. Finally, the gap lies along the angled edge with the finest mesh. The gap region ends $6.4 \mathrm{~mm}$ from the edge of the block because of a gap control piece that holds the gap space to the desired width. The mesh in the coolant channels is structured in the region near the walls. Overall, the CFD model contains 4.2 millions cells.

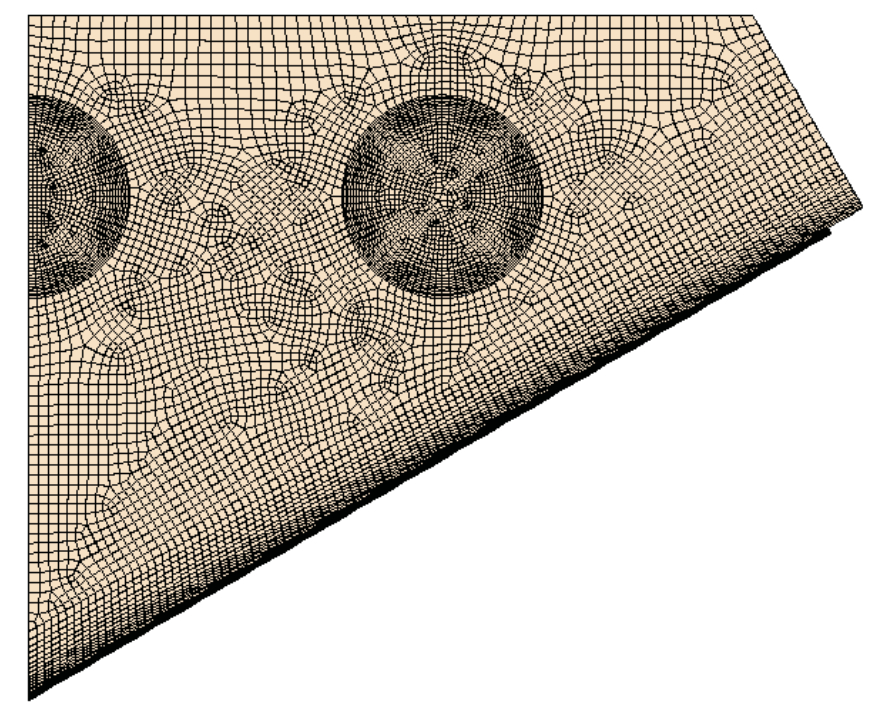

Figure 3-7 Plan view of the CFD model showing the mesh.

Figure 3-8 shows two views of the CFD model, including an isometric view of the whole model and a close-up view of the inlet and upper plenum. Because the MHTGR graphite blocks are beveled around their faces, the model includes this bevel. The close-up view of the upper plenum shows the beveled entrance into the gap region; the thin gap region extends downward from the bevel. The bottom of the upper fuel block and the top of the lower fuel block are also beveled.
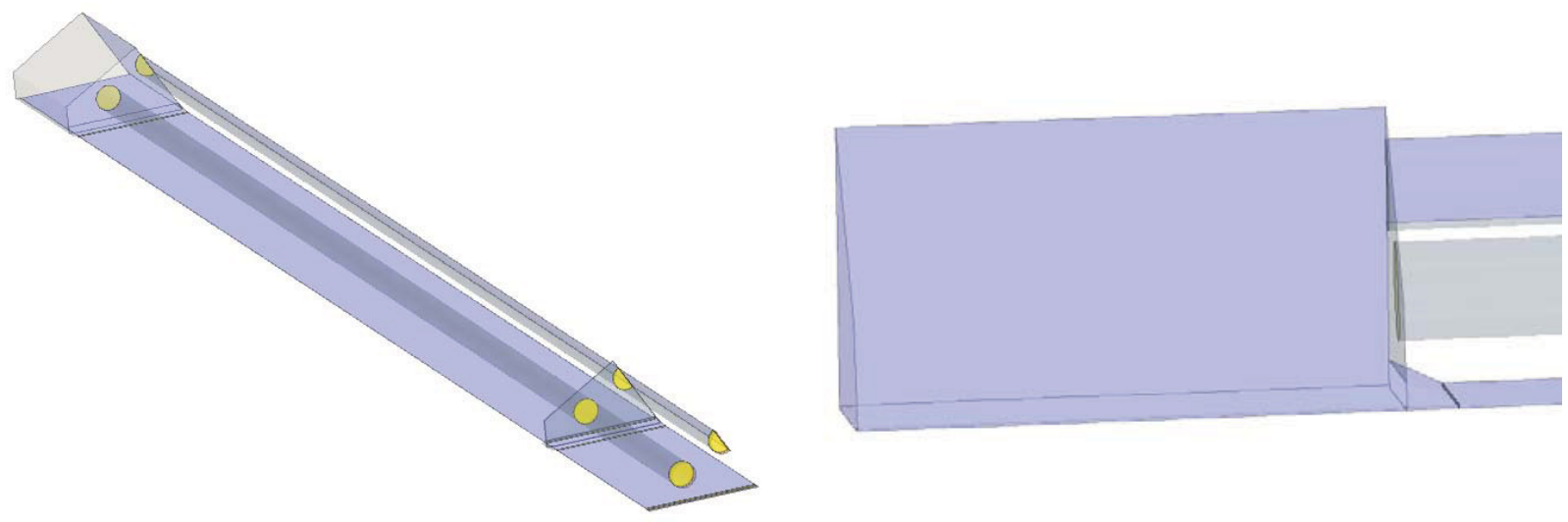

Figure 3-8. Overview of CFD model and close-up of upper plenum of model. 
The initial CFD model has a vertical gap of $2 \mathrm{~mm}$ and a horizontal gap of $2 \mathrm{~mm}$. The horizontal gap permits the flow in the coolant channels and the gap to readjust from flow in the upper section to the lower section. It is expected that the flow in the coolant channels will be turbulent while that in the gap regions will be laminar for the $2 \mathrm{~mm}$ case, transitional for the $4 \mathrm{~mm}$ case, and turbulent in the $10 \mathrm{~mm}$ case. This means that there concurrently will be turbulent flow in the channels and laminar flow in the gap for the $2 \mathrm{~mm}$ case. The commercial CFD code STARCCM+ v. 5.02.009 (see Appendix B) is used for the preliminary calculations. Note that this version is a later version than was used for the lower plenum standard problem in Section 2. Initially, the regions in the CFD model that represent the pressure-drop screens are left as open pathways because it is not known what the pressure drop in the upper fuel block will be.

The length of the coolant channels in the upper and lower fuel blocks, hereafter referred to as the first and second sections, respectively, are about 50 and 10 channel diameters. This means that the flow in the channel in the first section should become fully developed near the end, but not so in the second section. The length of the bypass gap in the first and second sections is 776 and 147 gap-widths, respectively. This means that the flow in the gap in both sections will become fully developed. Fully developed flow requires approximately 40 to 50 diameters in a cylindrical tube.

STARCCM+ has the capability to suppress turbulent flow in particular regions when there is turbulent flow specified in other regions. The Turbulence Suppression Model is used for the $2 \mathrm{~mm}$ vertical gap case along with the SKW turbulence model with the all $y^{+}$wall treatment. Turbulent flow is specified in the upper plenum, the coolant channels and the horizontal gap. The Turbulent Suppression Model is applied to the vertical gaps. Second order spatial discretization is used in the calculations. The inlet mass flow is estimated to be $17 \mathrm{~kg} / \mathrm{sec}$, based on the flow that can be obtained in the MIR facility using the current pump. This translates to $2.841 \mathrm{~kg} / \mathrm{sec}$ for the one-sixth sector of the MIR model. Pressure outlet boundary conditions are used for the channel and gap outlets. The mineral oil fluid properties used are density $=831.1 \mathrm{~kg} / \mathrm{m}^{3}$ and dynamic viscosity $=0.011685 \mathrm{~Pa}-\mathrm{sec}$. The 3-D flow is specified to be steady.

\subsection{Results and Discussion - 2 mm Gap}

Computations have been made for the $2 \mathrm{~mm}$ vertical gap case with a horizontal gap of $2 \mathrm{~mm}$. The flow in the gap region is suppressed to become laminar flow. Figure 3-9 plots the results for the velocity profile in the gap across the cross-section at about 580 channel widths (and is thus fully developed flow) below the inlet to the gap for the $2 \mathrm{~mm}$ horizontal gap case. The analytical solution for fully developed laminar flow between parallel plates is also plotted, based on the bulk velocity in the gap, calculated from the simulation. As can be seen, the profiles agree very well, verifying the CFD model for laminar flow. Additionally, the Reynolds number, about 30 based on the gap width, is well below the upper limit for laminar flow between parallel plates, which is 1,300 (Patel and Head 1969).

The Reynolds number for flow in the coolant channels, based on diameter, is computed to be about 9,600, which is well above the fully turbulent threshold of about 4,000 (Roberson and Crowe 1980). Hence, the application of a turbulence model to the flow in the coolant channels is appropriate. Turbulent flow is also applied in the upper plenum in the horizontal gap and in the beveled regions. 


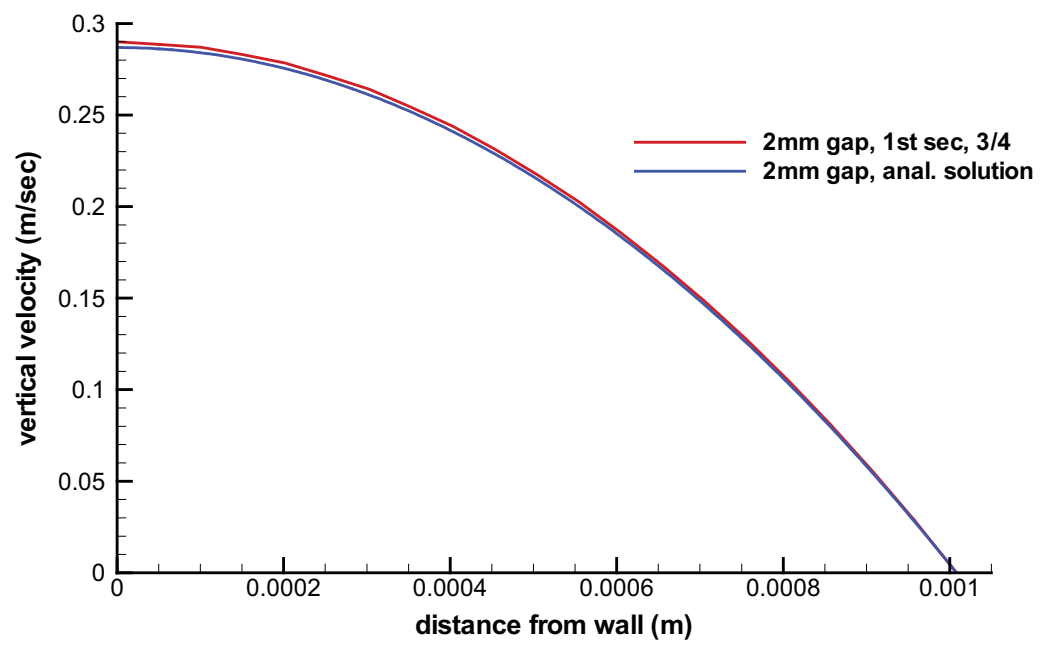

Figure 3-9. Velocity profile of flow in the gap compared to the analytical solution for laminar flow.

Figure 3-10 plots the mean vertical velocity in the coolant channel, first section, including at the entrance, at one-quarter, one-half, and three-quarters of the length of the channel and near the end of the first section channel where the length is about 48 channel diameters. The figure shows that the flow is essentially fully developed flow (unchanging from the three-quarters location to the $48 \mathrm{D}$ location) by the end of the first section. Figure 3-11 plots contours of the vertical velocity in the gap at the junction of the three blocks. The junction is at the lower left. Note that the width of the gap is $1 \mathrm{~mm}$. (There is a symmetric $1 \mathrm{~mm}$ on the other side of the symmetry boundary.) As can be seen, the maximum velocity in the gap junction is about $0.33 \mathrm{~m} / \mathrm{sec}$, an increase of about $10 \%$ from about $0.3 \mathrm{~m} / \mathrm{sec}$, which is the maximum velocity away from the junction. However, the area of the increased velocity is quite small, which indicates that the overall effect of the increase in velocity at the gap junctions will be small for the $2 \mathrm{~mm}$ vertical gap case.

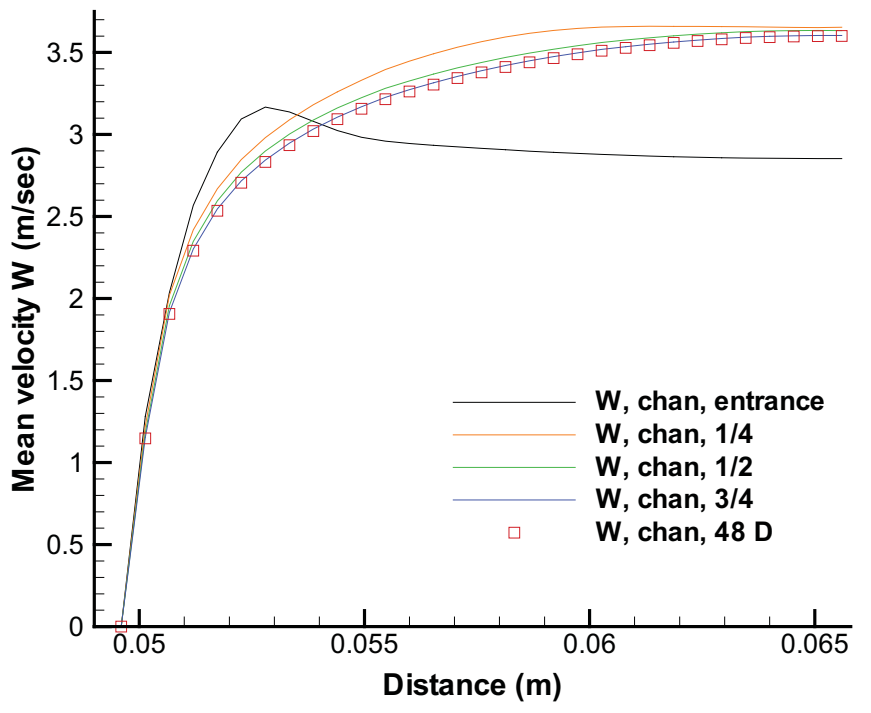

Figure 3-10. Turbulent velocity profiles in the coolant channel in the first section. 


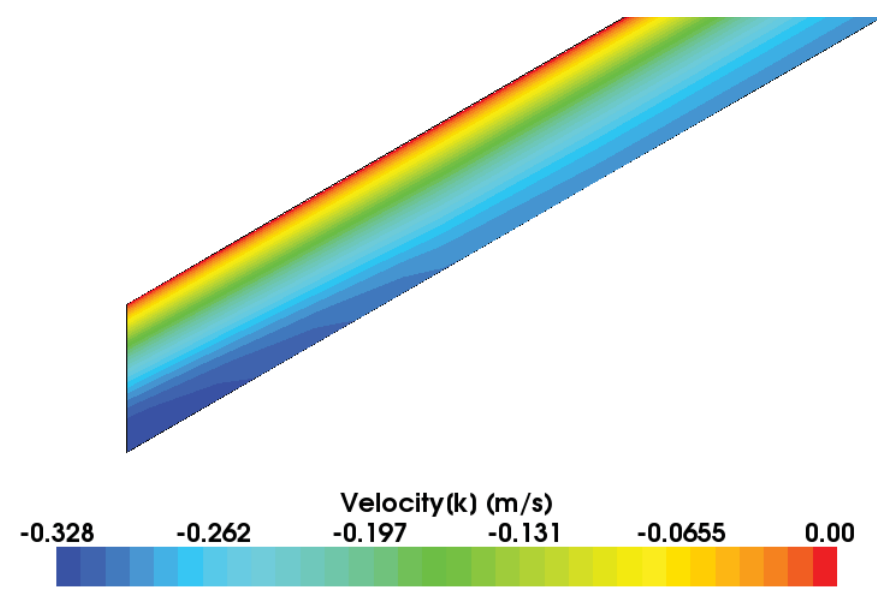

Figure 3-11. Vertical velocity contours in the gap at the junction of the three blocks.

Inasmuch as the inlet condition is specified as a constant mass flow, the pressure drop in the model is a function of the solution. The pressure drop affects the flow of the fluid, which can redistribute itself at the horizontal gap. Figure 3-12 plots the pressure drop across the CFD model for the gap and the whole coolant channel. The pressure is taken through the center axis of the coolant channel and along the plane of symmetry of the gap in its center. The pressure is required to be the same at the inlet and at the outlets, where it is set to zero gauge pressure. As shown, the pressure drops significantly near the entrance to the coolant channel, recovers, and then shows a lower gradient than seen for the gap region. In the second section, the pressure gradient is still steeper in the gap than in the coolant channel, but less steep than in the first section. This implies that the gap mass flow rate in the second section is less than in the first section. Indeed, the percent of total flow in the gap in the first section is $0.96 \%$ compared to $0.61 \%$ in the second section. It is obvious that resistance to flow is much higher in the gap than in the coolant channels. The total pressure drop in the CFD model is $11,640 \mathrm{~Pa}$; the drop in the first section is $10,400 \mathrm{~Pa}$.

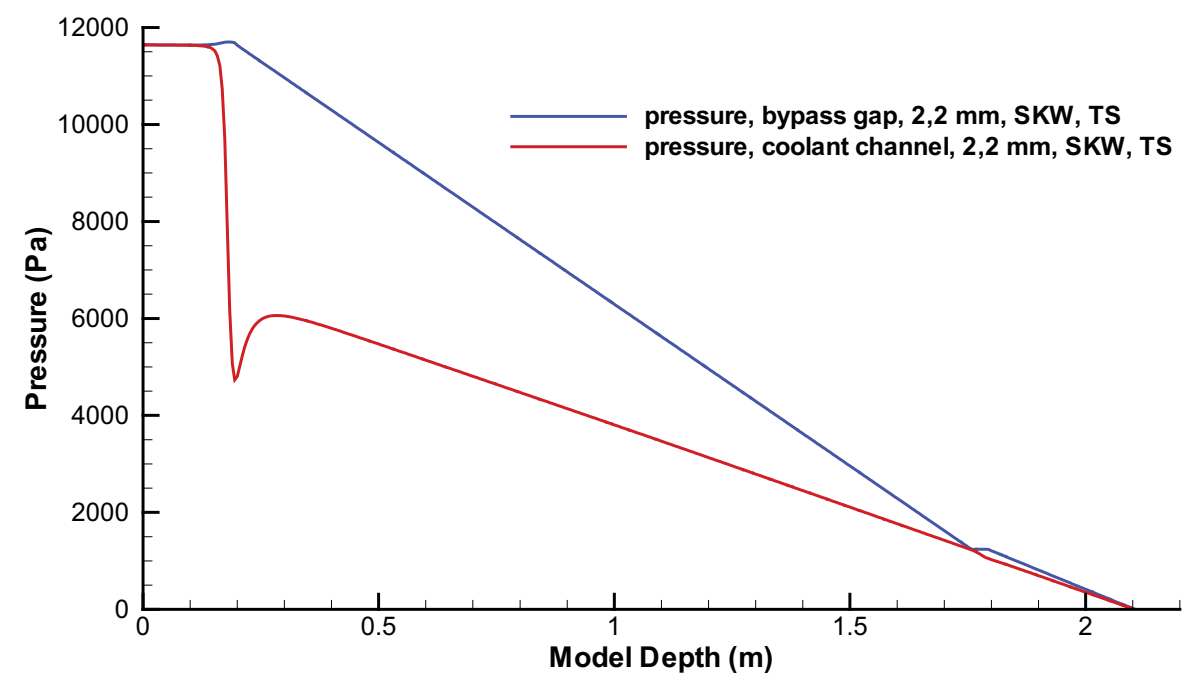

Figure 3-12. Axial pressure profiles through the full coolant channel and bypass flow gap. 
Figure 3-13 shows a contour plot of the pressure at the midpoint of the horizontal gap between the two sections. As can be seen, the pressure in the region of the gap is somewhat higher than in the region of the coolant channels. This implies that there is flow from the gap region toward the coolant channels, which is the case based on the computed flows in the gap for the first and second sections as discussed above. This higher pressure in the gap region is also seen in the first section (Figure 3-12). This is related to the higher resistance to flow shown by the bypass gap than for the coolant channels.

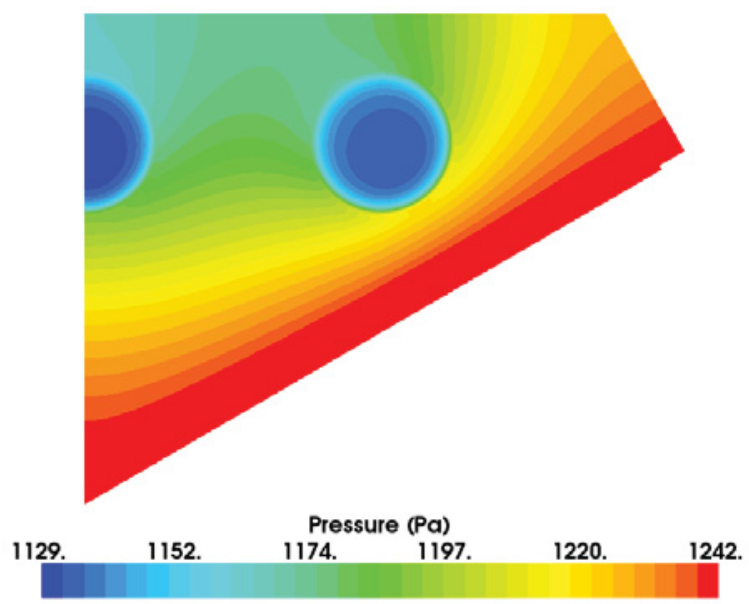

Figure 3-13. Pressure contours in the mid-plane of the horizontal gap.

Table 3-2 summarizes the inputs and results of the bypass flow simulation. As can be seen, the Reynolds number, based on channel diameter, for the coolant channel flow is around 9,600, well into the turbulent flow regime. The flow in the gap is clearly laminar, well below the limit of $R e=1300$. This validates the decision to use the turbulence suppression model for the gap flow. Finally, the percents of the total flow that appear in the gaps of the first and second sections are given. The flow in the second section gap is only about two-thirds that of the bypass flow in the first section.

Table 3-2. Inputs and results for the $2 \mathrm{~mm}$ vertical gap studies.

\begin{tabular}{|l|l|}
\hline Horizontal gap width $(\mathrm{mm})$ & 2 \\
\hline Total mass flow $(1 / 6$ sector $)(\mathrm{kg} / \mathrm{sec})$ & 2.841 \\
\hline \hline Re channel/flow regime $-1^{\text {st }}$ section & $9,610 /$ turbulent \\
\hline Re gap/flow regime $-1^{\text {st }}$ section & $31 /$ laminar \\
\hline Total pressure drop in model $(\mathrm{Pa})$ & 11640. \\
\hline Pressure drop in $1^{\text {st }}$ section $(\mathrm{Pa})$ & 10400. \\
\hline Gap flow fraction $-1^{\text {st }}$ section $(\%)$ & $0.96 \%$ \\
\hline Gap flow fraction $-2^{\text {nd }}$ section $(\%)$ & $0.61 \%$ \\
\hline
\end{tabular}

\subsection{Summary of the Bypass Flow Standard Problem}

A bypass flow model has been designed and is being constructed for installation in the INL's MIR test facility. The flow in the MIR facility is necessarily isothermal to achieve matching of the indices of refraction of the quartz used to construct the model and the mineral oil used as the working fluid; this provides significant optical advantages for the application of PIV that will be used to take the detailed velocity data. The model is designed to represent the gaps and some normal coolant channels near the junction of three hexagonal prismatic blocks of a prismatic VHTR. The model can be adjusted to have 
(vertical) gaps of 2, 4, and $10 \mathrm{~mm}$, representing 1,2, and $5 \mathrm{~mm}$ in the actual reactor core. (The scale factor is about two times larger than actual size.) Also, one full and one partial fuel blocks are included in the scaled model with an adjustable gap between them; the horizontal gap between the blocks can be either 2 or $10 \mathrm{~mm}$. Preliminary CFD calculations have been made for the case of a $2 \mathrm{~mm}$ vertical gap and a $2 \mathrm{~mm}$ horizontal gap. The CFD model applies a turbulence model in the flow in the model domain except in the gap, where turbulence is adequately suppressed to yield laminar flow by use of a turbulence suppression model available in the commercial CFD code (STARCCM+). The flow is shown to be turbulent in the normal coolant channels and laminar in the gap regions. The horizontal gap between the upper and lower fuel blocks allows the flow to readjust there. The gap flow is reduced in the lower section by about one third from that in the upper section, which is only $0.96 \%$ of the total flow. The flow resistance in the gap is clearly higher than the flow resistance in the coolant channels. 


\section{REFERENCES}

American Society of Mechanical Engineers (ASME), Quality Assurance Requirements for Nuclear Reactor Facility Applications, NQA-1 2000.

GA Technologies, Inc., 1992, Preliminary Safety Information Document for the Standard MHTGR, HTGR-86-024, Rev. 13.

General Atomics, 1977, Safety Analysis Report use of H451 Graphite in Fort St. Vrain Fuel Elements, GLP-5588.

Johnson, R. W. and H. Sato, 2010, Bypass Flow Computations using a One-Twelfth Symmetric Sector for Normal Operation in a 350 MWth Prismatic VHTR, Proceedings of High Temperature Reactor 2010 Conference, paper 152, Prague, Czech Republic, October 18-20, 2010.

Johnson, R. W., 2008a, "Development of a CFD Analysis Plan for the First VHTR Standard Problem," paper HTR2008-5828, Proceedings of the $4^{\text {th }}$ International Topical Meeting on High Temperature Reactor Technology (HTR2008), Washington, D.C., Sept. 28-Oct. 1.

Johnson, R. W., 2008b, "Modeling Strategies for Unsteady Turbulent Flows in the Lower Plenum of the VHTR," Nuclear Engineering \& Design, Vol. 238, pp. 482-491.

Johnson, R. W., 2009, "Examination of a Proposed Data Set Using CFD Calculations," paper FEDSM2009-78317, Proceedings of the ASME 2009 Fluids Engineering Summer Meeting (FEDSM2009), Vail, Colorado, August 2-5, 2009.

Johnson, R. W., 2010, "Evaluation of an experimental data set to be validation data for CFD for a VHTR," Proceedings of the CFD4NRS-3 conference, Washington, D.C., September 14-16, 2010.

Johnson, R. W., and H. M. McIlroy, 2010, "CFD Simulation of Proposed Validation Data for a Flow Problem Reconfigured To Eliminate an Undesirable Flow Instability," paper FEDSM-ICNMM201030522, Proceedings of the $3^{\text {rd }}$ Joint US-European Fluids Engineering Summer Meeting and $8^{\text {th }}$ International Conference on Nanochannels, Microchannels, and Minichannels, Montreal, Canada, August 1-5, 2010.

Johnson, R. W., and R. R. Schultz, 2009, Computational Fluid Dynamic Analysis of the VHTR Lower Plenum Standard Problem, INL/EXT-09-16325, Idaho National Laboratory, July 2009.

Johnson, R. W., H. M. McIlroy, R. C. Johnson, and D. P. Christensen, 2010, "Undesirable Flow Behavior in a Proposed Validation Data Set," Paper ICONE18-29474, Proceedings of the 18th International Conference on Nuclear Engineering (ICONE18), Xi'an, China, May 17-21 (2010).

MacDonald, P., J. Sterbentz, R. Sant, P. Bayless, R. Schultz, H. Gougar, R. Moore, A. Ougouag, and W. Terry, 2003, NGNP Preliminary Point Design Results of the Initial Neutronics and ThermalHydraulic Assessments, INEEL/EXT-03-00870.

McIlroy, H. M., D. M. McEligot, and R. J. Pink, 2008, "Measurement of Flow Phenomena in a Lower Plenum Model of a Prismatic Gas-Cooled Reactor," Proceedings of the $16^{\text {th }}$ International Conference on Nuclear Engineering (ICONE-16), Orlando, May 11-15, 2008.

Nakano, M., N. Tuji, and Y. Tazawa, 2008, "Conceptual Reactor Design Study of Very High Temperature Reactor (VHTR) with Prismatic-Type Core," Journal of Power and Energy Systems, Vol. 2, Issue 2, pp. 768-774.

Patel, V. C. and M. R. Head, 2006, "Some Observations on Skin Friction and Velocity Profiles in Fully Developed Pipe and Channel Flows," Journal of Fluid Mechanics, Vol. 38, pp. 181-201.

Roberson, J. A. and C. T. Crowe, 1980, Engineering Fluid Mechanics, $2^{\text {nd }}$ ed., Houghton Mifflin Co., Boston. 
Sato, H., R. W. Johnson, and R. R. Schultz, 2010, "Computational Fluid Dynamic Analysis of Core Bypass Flow Phenomena in a Prismatic VHTR," Annals of Nuclear Energy, Vol. 37, pp. 1172-1185.

Tak, N., M. Kim, and W. Lee, 2008, "Numerical investigation of a heat transfer within the prismatic fuel assembly of a very high temperature reactor," Annals of Nuclear Energy, Vol. 35, pp. 1892-1899.

Takada, E., S. Nakagawa, N. Fujimoto, and D. Tochio, 2004, “Core Thermal-Hydraulic Design,” Nuclear Engineering and Design, Vol. 233, pp. 37-43. 
Appendix A

Standard Model Problem Geometry 


\section{Appendix A-Lower Plenum Standard Problem Model Geometry}

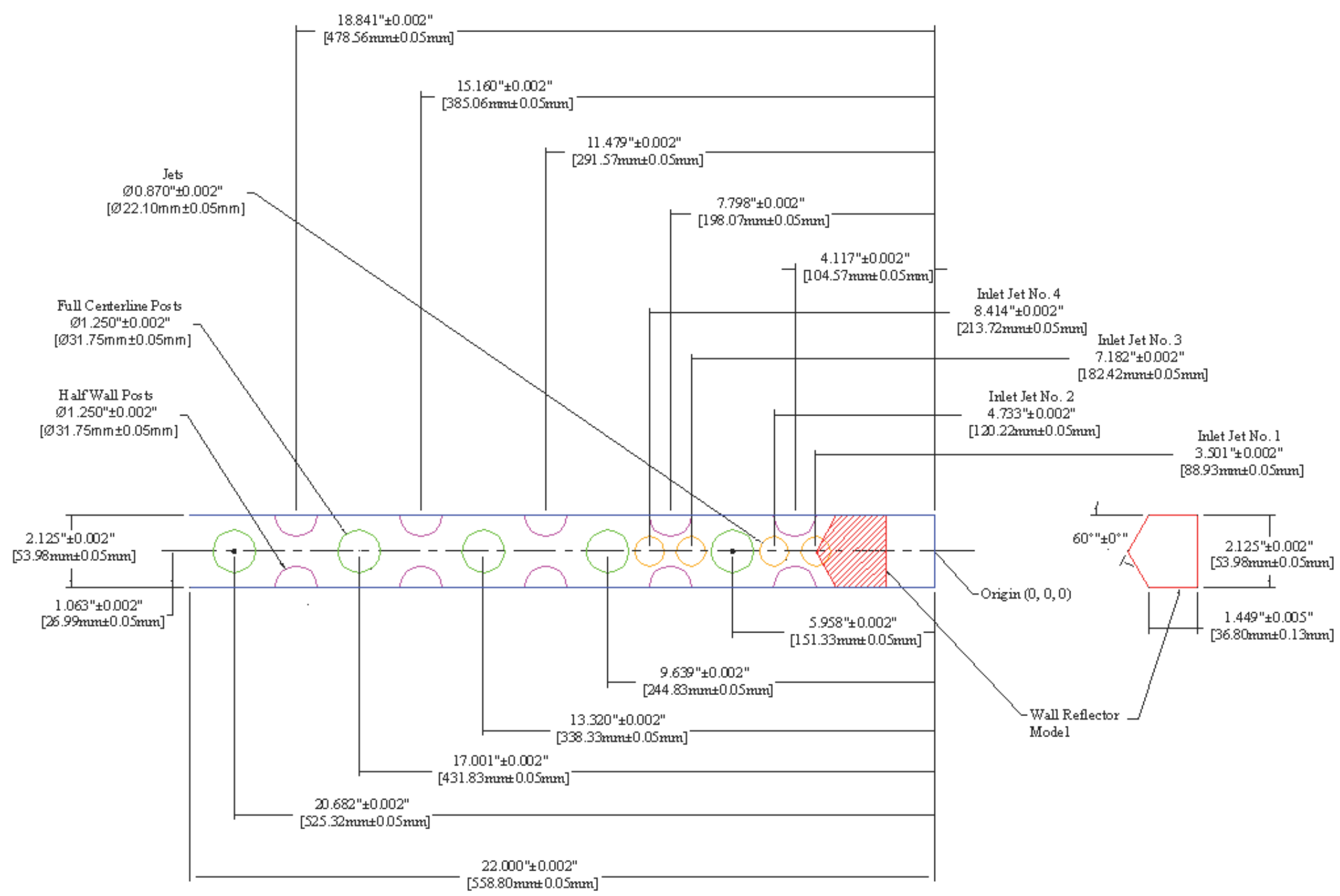

Figure A-1. Top view of standard problem model geometry. 


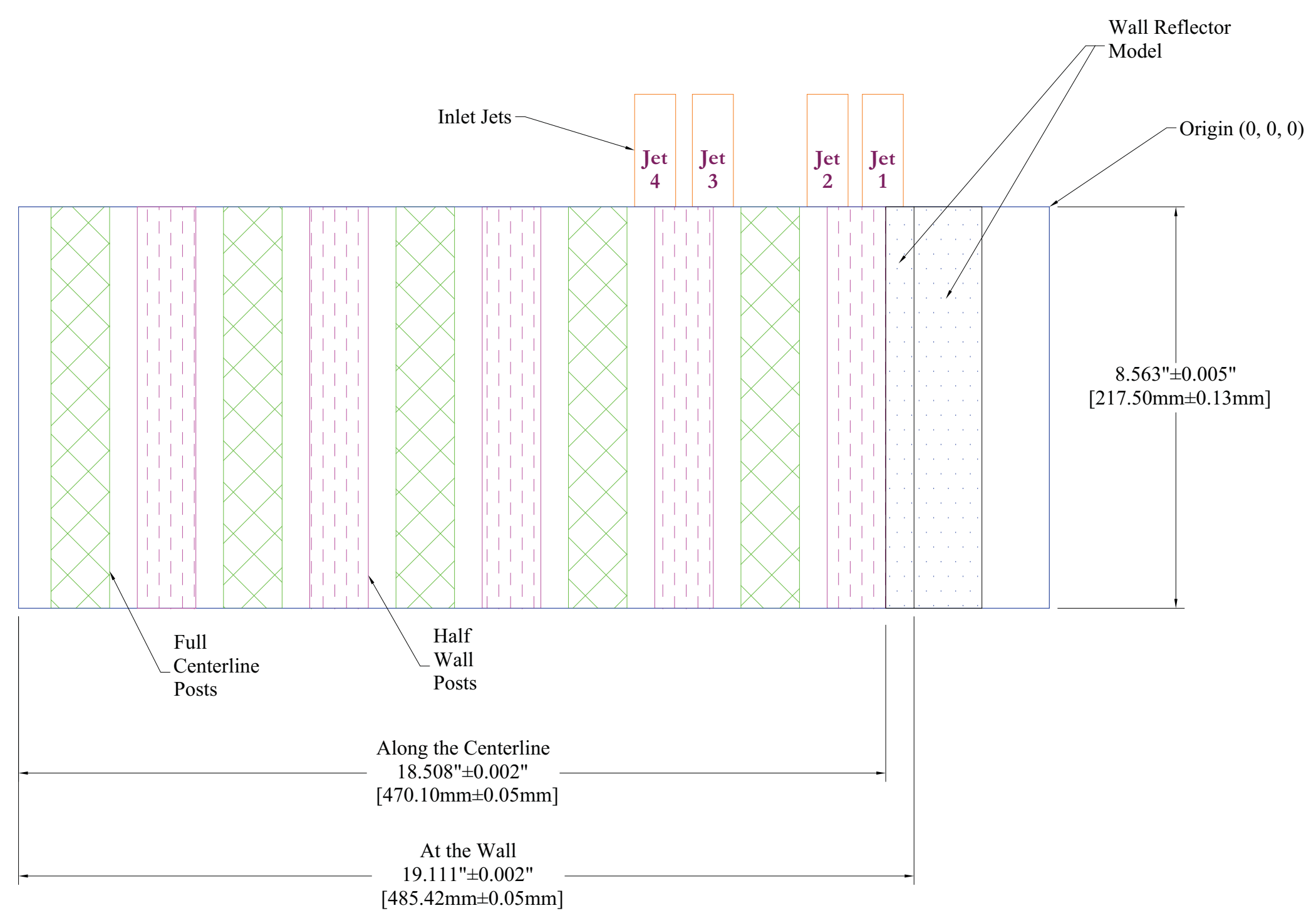

Figure A-2. Front view of standard problem model geometry. 
Appendix B

Computer Code Quality Assurance 


\section{Appendix B-Computer Code Quality Assurance}

Table B-1. Quality assurance information for the computer codes used for the computer simulations.

\begin{tabular}{|c|c|c|c|c|c|}
\hline $\begin{array}{l}\text { Computer } \\
\text { Code }\end{array}$ & Version & Vendor & V\&V Status & INL Computer & $\begin{array}{c}\text { Computer } \\
\text { Operating } \\
\text { System } \\
\end{array}$ \\
\hline FLUENT & 6.3 .26 & $\begin{array}{l}\text { FLUENT Inc./Ansys } \\
\text { Inc., } 10 \text { Cavendish } \\
\text { Court, Centerra } \\
\text { Resource Park, } \\
\text { Lebanon, NH, } 03766 \\
\text { USA }\end{array}$ & $\begin{array}{l}\text { Vendor holds } \\
\text { ISO } 9001 \\
\text { certification }\end{array}$ & $\begin{array}{c}\text { Dell PowerEdge } \\
1950 \text { distributed } \\
\text { memory cluster } \\
\text { (Helios) }\end{array}$ & $\begin{array}{c}\text { OpenSUSE } \\
11.1\end{array}$ \\
\hline GAMBIT & 2.4 .6 & $\begin{array}{l}\text { FLUENT Inc./Ansys } \\
\text { Inc., } 10 \text { Cavendish } \\
\text { Court, Centerra } \\
\text { Resource Park, } \\
\text { Lebanon, NH, } 03766 \\
\text { USA } \\
\end{array}$ & $\begin{array}{l}\text { Vendor holds } \\
\text { ISO } 9001 \\
\text { certification }\end{array}$ & $\begin{array}{c}\text { Dell PowerEdge } \\
1950 \text { distributed } \\
\text { memory cluster } \\
\quad \text { (Helios) }\end{array}$ & $\begin{array}{c}\text { OpenSUSE } \\
11.1\end{array}$ \\
\hline STARCCM+ & 3.04 .20 & $\begin{array}{c}\text { CD-adapco, } 60 \\
\text { Broadhollow Road, } \\
\text { Melville, NY } 11747\end{array}$ & $\begin{array}{l}\text { Vendor holds } \\
\text { ISO } 9001 \\
\text { certification }\end{array}$ & $\begin{array}{c}\text { SGI Altix ICE } \\
8200 \text { distributed } \\
\text { memory blade } \\
\text { cluster } \\
\text { (Ice Storm) }\end{array}$ & $\begin{array}{c}\text { SUSE Linux } \\
\text { Enterprise } \\
\text { Server } 10 \\
\text { Service Pack } \\
\text { 2, with SGI } \\
\text { Propack } 6\end{array}$ \\
\hline STARCCM+ & 5.02 .009 & $\begin{array}{c}\text { CD-adapco, } 60 \\
\text { Broadhollow Road, } \\
\text { Melville, NY } 11747\end{array}$ & $\begin{array}{l}\text { Vendor holds } \\
\text { ISO } 9001 \\
\text { certification }\end{array}$ & $\begin{array}{c}\text { SGI Altix ICE } \\
8200 \text { distributed } \\
\text { memory blade } \\
\text { cluster } \\
\text { (Ice Storm) }\end{array}$ & $\begin{array}{c}\text { SUSE Linux } \\
\text { Enterprise } \\
\text { Server } 10 \\
\text { Service Pack } \\
\text { 2, with SGI } \\
\text { Propack } 6\end{array}$ \\
\hline
\end{tabular}

